\title{
LYON NATURAL RADIOCARBON MEASUREMENTS X
}

\section{JACQUES EVIN, JOELLE MARECHAL, and GERARD MARIEN}

Laboratoire de radiocarbone, Centre de Datation et d'Analyses isotopiques, Université Claude-Bernard de Lyon

43 boulevard du 11 Novembre 1918, 69622 Villeurbanne, France

\section{INTRODUCTION}

This list includes most of the measurements made since the beginning of the compilation of our previous list ( $R, 1983$, v 25, p 59-126), in 1982 and 1983, as well as some earlier measurements of extended geologic or archaeologic studies.

Three ml benzene samples were measured by the liquid scintillation process. When the amount of available carbon was too low, inactive benzene was added; dilution ratio (dr) is the amount of active benzene in the total $3 \mathrm{ml}$. Pretreatment, treatment, and counting procedures remain as previously described (R, 1983, v 25, p 59).

Calculations followed international rules $\left({ }^{14} \mathrm{C}\right.$ half-life $=5570 \pm 0$; age error is based on one standard deviation of contemporary standard, background, and sample counts). An isotopic fractionation correction is made only for bones $(+80 \mathrm{yr})$. The published intervals of corrected dates for samples younger than 7250 BP are derived from Klein et al (1982) and appear under the BP date with an asterisk $(*)$.

\section{ACKNOWLEDGMENTS}

We would like to thank Gerard Drevon for routine preparation of most samples. We are indebted to Claude-Bernard University for administrative and partial financial support, and are grateful to Christiane Pachiaudi and to the staff of the Nuclear Physics Institute for technical assistance. Finally, we thank Patricia Anderson-Gerfaud, CNRS, Maison de l'Orient, Lyon, for revision of the English text.

\section{GEOLOGIC SAMPLES}

Samples from Peat Bogs

\section{Ly-2852. Coron, Belley, Ain}

$630 \pm 160$

$*_{A D} 1200-1485$

Peat from base of small bog $\left(45^{\circ} 45^{\prime} \mathrm{N}, 5^{\circ} 31^{\prime} \mathrm{E}\right)$, coll 1981 and subm 1982 by J P Bravard, Lab Geog, Univ Lyon III. Comment (JPB): as peat bog overlies settlement of Gallo-Roman period or High Middle ages, date proves that peat developed after several centuries and might correspond to "Little Ice Age."

\section{Ly-2851. Le Grand Marais, Les Avenières, Isère}

$$
1260 \pm 170
$$

Peat from base of bog in former bed of Rhône $\mathrm{R}\left(45^{\circ} 36^{\prime} \mathrm{N}, 5^{\circ} 31^{\prime} \mathrm{E}\right)$. Coll and subm 1983 by J P Bravard ( $0.7 \mathrm{dr}$ ). Comment (JPB): date agrees perfectly with expected age and results from Brégnier-Cordon and Cham- 
pagneux (below). It confirms that development of bog began just after change in course of Rhône $\mathrm{R}$ which probably occurred during 6 th century (Bravard, 1983).

\section{Ly-2514. La Touvière, Massignieu de Rives, Ain}

$2020 \pm 120$

*375BC-AD215

Wood from lacustrine clay outcropping on side of Bart Lake $\left(45^{\circ} 46^{\prime} \mathrm{N}, 5^{\circ} 45^{\prime} \mathrm{E}\right)$. Coll and subm 1981 by R Vilain, Lab Geol, Univ Lyon I. Comment (RV): date is much younger than expected and proves that wood was brought in by recent colluvia.

\section{Marais de Landes series, Saint-Loup, Charente-Maritime}

Samples from three levels of Boring P4 in marsh $\left(46^{\circ} 10^{\prime} \mathrm{N}, 0^{\circ} 38^{\prime} \mathrm{W}\right)$. Coll and subm 1982 by M Mazeau, Fr Geophys Prospection Cie, Limoges.

General Comment (MM): dates show that peat development recently finished. Because clays ("Le Bri”) embedding wood (Ly-2692) are of marine origin and may correspond to max of Flandrian transgression, Ly-2691 proves there was relatively long sedimentation hiatus before peat formation.

TABLE 1

Marais de Landes

\begin{tabular}{|c|c|c|c|c|c|}
\hline $\begin{array}{c}\text { Sample } \\
\text { no. }\end{array}$ & $\begin{array}{l}\text { Depth } \\
\text { (m) }\end{array}$ & Sample & $\mathrm{DR}$ & $\begin{array}{c}\text { Age } \\
\mathrm{BP}\end{array}$ & $\begin{array}{c}\text { Corrected } \\
\text { date } \\
\text { interval }\end{array}$ \\
\hline Ly-2690 & $0.0-0.1$ & "Surface" peat & 1 & $640 \pm 120$ & AD $1235-1415$ \\
\hline-2691 & $3.0-3.1$ & Basal peat & 0.7 & $5130 \pm 180$ & $4395-3635 \mathrm{BC}$ \\
\hline-2692 & $3.1-3.2$ & Wood from clay & 0.7 & $8720 \pm 210$ & \\
\hline
\end{tabular}

\section{Ly-2999. Ichtratzheim, Bas-Rhin}

$4960 \pm 150$

Peat with wood debris interbedded in alluvia of former bed of Ill $\mathrm{R}$ $\left(48^{\circ} 27^{\prime} \mathrm{N}, 7^{\circ} 41^{\prime} \mathrm{E}\right)$. Coll and subm 1983 by A Schnitzler, Lab Bot, Univ Strasbourg. Comment (AS): date is much older than expected for $\mathrm{N}$ extension of Ill R bed, because of geomorphol and hist data; Ill R was assumed to have joined Rhine $\mathrm{R}$ much further $\mathrm{S}$ in Middle ages.

\section{Marais de Brière series, Loire Atlantique}

Samples from several levels in two borings drilled as part of PICG $158 \mathrm{~b}$ "Lake and Sea environment" proj at Le Butteau-Piquet, near SaintLyphard $\left(47^{\circ} 23^{\prime} \mathrm{N}, 2^{\circ} 17^{\prime} \mathrm{W}\right)$ and Penlys $\left(47^{\circ} 26^{\prime} \mathrm{N}, 2^{\circ} 14^{\prime} \mathrm{W}\right)$ near La Chapelle des Marais. Coll and subm 1982 by L Visset, Lab Ecol, Univ Nantes.

General Comment (LV): all dates are a little older than expected. They confirm Sub-boreal age of dated level and regression from edges of former Brière gulf. They may be compared with two other unpub results from same peat bog: Ny-682: $4330 \pm 75$ in Errand I. (Visset, 1982) from level comparable to that from Le Butteau-Piquet (Ly-2903), and Ny-523: $4120 \pm 90$ in Canal des Fougères from fossilized tree trunk as Ly-2904. 
TABLE 2

Marais de Brière

\begin{tabular}{|c|c|c|c|c|c|c|c|}
\hline $\begin{array}{c}\text { Sample } \\
\text { no. }\end{array}$ & Boring & $\begin{array}{l}\text { Level } \\
(\mathrm{cm})\end{array}$ & $\begin{array}{l}\text { Pollen } \\
\text { phase }\end{array}$ & Sample & $\begin{array}{l}\text { Expected } \\
\text { age } \\
\text { (BP) }\end{array}$ & $\begin{array}{c}\text { Age } \\
(\mathrm{BP})\end{array}$ & $\begin{array}{l}\text { Corrected } \\
\text { date } \\
\text { interval } \\
\text { (BC) }\end{array}$ \\
\hline Ly-2906 & Penlys & $130-140$ & $\begin{array}{l}\text { End of } \\
\quad \text { Sub-boreal }\end{array}$ & Peat and wood & ca3600 & $4440 \pm 120$ & $3480-2985$ \\
\hline-2905 & Penlys & $142-148$ & $\begin{array}{l}\text { Middle of } \\
\text { Sub-boreal }\end{array}$ & Peaty clay & ca4150 & $4680 \pm 110$ & $3765-3160$ \\
\hline-2904 & Penlys & 145 & $\begin{array}{l}\text { Middle of } \\
\text { Sub-boreal }\end{array}$ & $\begin{array}{l}\text { Fragment of } \\
\text { tree trunk }\end{array}$ & ca4150 & $4710 \pm 140$ & $3795-3150$ \\
\hline-2903 & $\begin{array}{r}\text { Butteau- } \\
\text { Piquet }\end{array}$ & $222-228$ & $\begin{array}{l}\text { Beginning of } \\
\text { Sub-boreal }\end{array}$ & Peaty clay & ca4300 & $5010 \pm 130$ & $4110-3395$ \\
\hline
\end{tabular}

\section{Marais de Syl series, Lavau-sur-Loire, Loire Atlantique}

Peat from four levels in boring at "Pré du Fauchais" in bog $\left(47^{\circ} 20^{\prime} \mathrm{N}\right.$, $1^{\circ} 56^{\prime} \mathrm{W}$ ). Coll and subm 1982 by D Voeltzel and L Visset.

General Comment (LV): all dates are much older than expected. They confirm that base of boring (Ly-2907) belongs to Atlantic and upper level (Ly2910) dates to Sub-boreal. Discrepancies between results and palynol data and results from La Grande Brière peat bog (above) remain unexplained and need further confirmation from other borings.

TABLE 3

Marais de Syl

\begin{tabular}{|c|c|c|c|c|c|}
\hline $\begin{array}{c}\text { Sample } \\
\text { no. }\end{array}$ & $\begin{array}{l}\text { Level } \\
(\mathrm{m})\end{array}$ & $\begin{array}{l}\text { Pollen } \\
\text { phase }\end{array}$ & $\begin{array}{c}\text { Expected } \\
\text { age } \\
\text { (BP) }\end{array}$ & $\begin{array}{l}\text { Age } \\
\text { (BP) }\end{array}$ & $\begin{array}{c}\text { Corrected } \\
\text { date } \\
\text { interval } \\
\text { (BC) }\end{array}$ \\
\hline Ly-2910 & $2-2.08$ & $\begin{array}{l}\text { Sub-atlantic/ } \\
\text { Sub-boreal } \\
\text { limit }\end{array}$ & 3000 & $4070 \pm 130$ & $3010-2305$ \\
\hline-2909 & $4.42-4.52$ & $\begin{array}{l}\text { Middle of } \\
\text { Sub-boreal }\end{array}$ & 4500 & $5680 \pm 140$ & $4920-4145$ \\
\hline-2908 & $5.05-5.15$ & $\begin{array}{l}\text { Beginning of } \\
\text { Sub-boreal }\end{array}$ & 5000 & $5840 \pm 140$ & $5185-4425$ \\
\hline-2907 & $5.45-5.53$ & $\begin{array}{l}\text { Sub-boreal/ } \\
\text { Atlantic } \\
\text { limit }\end{array}$ & 5700 & $6010 \pm 140$ & $5265-4560$ \\
\hline
\end{tabular}

\section{Le Marais series, Barrou, Indre-et-Loire}

Wood from two depths of peaty levels of fill of former channel $\left(46^{\circ} 53^{\prime}\right.$ $\mathrm{N}, 0^{\circ} 44^{\prime} \mathrm{E}$ ). Some levels contain well-known Grand Pressigny flint artifacts manufactured locally and assoc with ceramics of Bronze or Neolithic age. Coll 1979 by "Les Amis du Grand Pressigny" Soc and subm 1980 by J Allain and J Despriée, Dir Antiquités Prehist Orléans.

Ly-2307. Le Marais F6

$7340 \pm 100$

From upper level, 1.2 to $1.5 \mathrm{~m}$ depth. 


\section{Ly-2308. Le Marais F8}

$7010 \pm 150$

From lower level, 2 to $2.3 \mathrm{~m}$ depth.

General Comment (JD): dates are close in age and similar to those obtained for other peaty fills in region which were also formed during Atlantic period corresponding to Neolithic. They show that Bronze age remains are intrusive.

\section{Le Pré Maudit series, Gathemo, Manche}

Peat from several levels of $\mathrm{N} 400$ boring of bog $\left(48^{\circ} 45^{\prime} \mathrm{N}, 1^{\circ} 37^{\prime} \mathrm{W}\right)$. Coll and subm 1983 by C Lechevalier and L Barthélémy, Lab Geog Phys, Univ Paris X, Nanterre; these samples complete previous series (R, 1983, v 25, p 64) (Lechevalier, 1983).

General Comment (CL): all results are within expected range and confirm previously dated series, particularly for beginning of site deposits. Ly-3014 suggests that Ly-2046: $9250 \pm 180$ may be a little too old due to local colluvia of gray clay deposited during Pre-boreal before peat development.

TABLE 4

Le Pré Maudit

\begin{tabular}{|c|c|c|c|c|c|}
\hline $\begin{array}{c}\text { Sample } \\
\text { no. }\end{array}$ & $\begin{array}{l}\text { Depth } \\
\text { (cm) }\end{array}$ & $\begin{array}{c}\text { Pollen phase and } \\
\text { expected climatic } \\
\text { phase }\end{array}$ & $\mathrm{DR}$ & $\begin{array}{l}\text { Age } \\
\text { (BP) }\end{array}$ & $\begin{array}{l}\text { Corrected } \\
\text { date } \\
\text { interval } \\
\text { (BC) }\end{array}$ \\
\hline Ly-3012 & $317-326$ & VIIa: Atlantic & 0.5 & $6580 \pm 170$ & $5825-5215$ \\
\hline-3013 & $329-336$ & $\begin{array}{l}\text { VIIa: Beginning of } \\
\text { Atlantic }\end{array}$ & 1 & $7110 \pm 150$ & $6465-5480$ \\
\hline-3014 & $341-345$ & $\mathrm{~V}:$ 1st part of Boreal & 0.8 & $8560 \pm 190$ & \\
\hline-3015 & $350-358$ & IV: End of Pre-boreal & 0.7 & $9120 \pm 200$ & \\
\hline
\end{tabular}

\section{Vallat-Neuf series, Berre l'Etang, Bouches-du-Rhône}

Clay with low organic content from three levels of two borings in bog on N side of Etang de Berre gulf $\left(43^{\circ} 29^{\prime} \mathrm{N}, 5^{\circ} 13^{\prime} \mathrm{E}\right)$. Coll 1981 and subm 1982 by H Triat-Laval, Lab Palynol, Univ Aix-Marseille III.

Ly-2685. Vallat-Neuf 180-190

$1770 \pm 310$

From 180 to $190 \mathrm{~cm}$ depth in D4 boring $(0.07 \mathrm{dr})$. Pollen diagram shows Sub-atlantic phase during the extension of olive-tree (Olea sp) cultivation. Comment (HTL): date confirms that extension of olive cultivation occurred earlier in Provence region than in neighboring $\mathrm{W}$ Languedoc region where it was dated at Mauguio site, MC-1404: $1300 \pm 60$ (Planchais, 1982).

Ly-2686. Vallat-Neuf 200-205

$2090 \pm 200$

*565BC-AD235

From 200 to $205 \mathrm{~cm}$ depth in D4 boring $(0.3 \mathrm{dr})$. Pollen diagram shows beginning of continuous curve of Olea. Comment (HTL): date attributes 
beginning of olive cultivation to Historic period following Protohistoric precultivation period (Triat-Laval, 1982).

Ly-2650. Vallat-Neuf 355-362

$7340 \pm 270$

From 355 to $362 \mathrm{~cm}$ depth near base of D3 boring. Pollen diagram suggests beginning of Atlantic climatic phase with thermophilic vegetation and extension of Quercus forest. Comment (HTL): date confirms expected age and agrees with previous data from W Provence, eg, at Mollègès (R, 1978, v 20, p 20) (Triat-Laval, 1979).

\section{L'Alpe d'Huez series, Isère}

Wood from two peaty outcroppings in ski sta $\left(45^{\circ} 6^{\prime} \mathrm{N}, 6^{\circ} 4^{\prime} \mathrm{E}\right)$. Coll and subm by $\mathrm{M}$ Chardon, Lab Geog, Univ Grenoble.

\section{Ly-2696. Alpes d'Huez, Le Rif}

$3540 \pm 160$

*2305-1640BC

From 1.5m depth in small bog near cablecar sta. Coll and subm 1981.

\section{Ly-1967. Alpes d'Huez, Le Lynx}

$5090 \pm 150$

$* 4315-3645 B C$

From $0.8 \mathrm{~m}$ depth at top of peaty and clayey layer in center of village. Coll and subm 1978.

General Comment (MC): both dates indicate that peat formation is relatively old. They also show presence of forest at alt, ca 1800 to $2000 \mathrm{~m}$ in Les Rousses massif at end of Atlantic and beginning of Sub-boreal, ie, several hundred $\mathrm{m}$ higher than present timber line in region.

\section{Bonnecombe series, Salces, Lozère}

Peat from several levels of bog near Los Pesquio $\left(44^{\circ} 34^{\prime} \mathrm{N}, 3^{\circ} 8^{\prime} \mathrm{E}\right)$. Coll 1980 and subm 1982 by J L de Beaulieu and M Reille, Lab Palynol, Univ Aix-Marseille III.

TABLE 5

Bonnecombe

\begin{tabular}{|c|c|c|c|c|c|c|}
\hline $\begin{array}{c}\text { Sample } \\
\text { no. }\end{array}$ & $\begin{array}{l}\text { Depth } \\
\text { (cm) }\end{array}$ & Pollen event & $\begin{array}{l}\text { Climatic } \\
\text { phase }\end{array}$ & DR & $\begin{array}{l}\text { Age } \\
(\mathrm{BP})\end{array}$ & $\begin{array}{c}\text { Corrected } \\
\text { date } \\
\text { interval }\end{array}$ \\
\hline Ly-2558 & $20-30$ & $\begin{array}{l}\text { Fagus decrease, } \\
\text { presence of } \\
\text { cereals }\end{array}$ & Sub-atlantic & 0.7 & $2220 \pm 210$ & $775 \mathrm{BC}-\mathrm{AD} 205$ \\
\hline-2559 & $105-112$ & Beginning of Fagus & $\begin{array}{l}\text { End of } \\
\text { Atlantic }\end{array}$ & 0.7 & $5150 \pm 210$ & $4405-3645 \mathrm{BC}$ \\
\hline-2560 & $200-210$ & Beginning of Tilia & $\begin{array}{l}\text { Beginning } \\
\text { of Atlantic }\end{array}$ & 0.5 & $6910 \pm 170$ & $6215-5385 \mathrm{BC}$ \\
\hline-2561 & $285-290$ & $\begin{array}{l}\text { Beginning of } \\
\text { Corylus }\end{array}$ & Pre-boreal & 0.3 & $9500 \pm 240$ & \\
\hline-2562 & $300-307$ & Betula & Pre-boreal & 0.2 & $9180 \pm 290$ & \\
\hline-2563 & $335-345$ & & Pre-boreal & 0.5 & $10240 \pm 210$ & \\
\hline-2564 & $355-360$ & Pinus & $\begin{array}{c}\text { Beginning } \\
\text { of Pre- } \\
\text { boreal }\end{array}$ & 0.2 & $10640 \pm 300$ & \\
\hline
\end{tabular}


General Comment (JLdeB): except for Ly-2564, which is ca 1500 yr too old for unknown reason, all values are in expected range suggested by pollen diagram. They all agree with previously pub series from peat bog in region (de Beaulieu, Pons, \& Reille, in press), eg, Ly-2560 is contemporaneous with Ly-2605: $6990 \pm 160$ from Brameloup and Ly-2112: $6880 \pm 200$ from Chaumette bogs.

\section{Cheylane series, Laveissenet, Cantal}

Peat from several levels of bog presently submerged in pond at alt $1040 \mathrm{~m}$ on La Planèze de Saint-Flour plateau ( $\left.45^{\circ} 10^{\prime} \mathrm{N}, 2^{\circ} 53^{\prime} \mathrm{E}\right)$. Coll 1979 and subm 1980 by $\mathrm{J} \mathrm{L}$ de Beaulieu.

General Comment (JLdeB): almost all dates agree with expected ages, except Ly-2071, which, because of large statistical margin, is ca 800 yr too old; Ly-2072 is also ca $1000 \mathrm{yr}$ too old for unknown reason. Ly-2068 indicates much older age than in other pollen diagram from region for extension of Abies (de Beaulieu, Pons, \& Reille, in press).

TABLE 6

Cheylane

\begin{tabular}{|c|c|c|c|c|c|c|}
\hline $\begin{array}{c}\text { Sample } \\
\text { no. }\end{array}$ & $\begin{array}{l}\text { Depth } \\
(\mathrm{cm})\end{array}$ & Pollen event & $\begin{array}{c}\text { Climatic } \\
\text { phase }\end{array}$ & DR & $\begin{array}{c}\text { Age } \\
\text { (BP) }\end{array}$ & $\begin{array}{c}\text { Corrected } \\
\text { date } \\
\text { interval } \\
\text { (BC) }\end{array}$ \\
\hline Ly-2067 & $80-90$ & Extension of Fagus & Sub-boreal & 0.7 & $4180 \pm 160$ & $3335-2410$ \\
\hline-2068 & $130-140$ & Extension of Abies & $\begin{array}{l}\text { Middle of } \\
\text { Atlantic }\end{array}$ & 1 & $5560 \pm 170$ & $4710-3960$ \\
\hline-2069 & $170-180$ & Appearance of Tilia & Atlantic & 0.8 & $6790 \pm 190$ & $6125-5270$ \\
\hline-2070 & $220-230$ & Decrease of Corylus & Boreal & 1 & $8220 \pm 200$ & \\
\hline-2071 & $250-260$ & Extension of Corylus & Pre-boreal & 0.2 & $7870 \pm 360$ & \\
\hline-2108 & $260-270$ & Extension of Corylus & Pre-boreal & 0.7 & $8930 \pm 180$ & \\
\hline-2109 & $280-290$ & $\begin{array}{l}\text { Appearance of } \\
\text { Corylus }\end{array}$ & Pre-boreal & 0.2 & $9250 \pm 350$ & \\
\hline-2072 & $320-330$ & Appearance of Betula & $\begin{array}{l}\text { Beginning of } \\
\text { Alleröd }\end{array}$ & 0.8 & $10440 \pm 220$ & \\
\hline
\end{tabular}

\section{Suc du Lac d'En Bas series, La Godivelle, Puy de Dôme}

Gyttja with low organic content from three levels of bog at $1260 \mathrm{~m}$ alt, Le Cézalier massif $\left(45^{\circ} 23^{\prime} \mathrm{N}, 2^{\circ} 55^{\prime} \mathrm{E}\right)$. Coll and subm 1982 by $\mathrm{M}$ Reille and $\mathrm{J} \mathrm{L}$ de Beaulieu. Samples dated to complete data from three previous borings from region $(\mathrm{R}, 1983$, v 25, p 67).

TABLE 7

Suc du Lac d'En Bas

\begin{tabular}{|c|c|c|c|c|}
\hline $\begin{array}{c}\text { Sample } \\
\text { no. }\end{array}$ & $\begin{array}{c}\text { Depth } \\
(\mathrm{cm})\end{array}$ & $\begin{array}{c}\text { Expected } \\
\text { climatic } \\
\text { phase }\end{array}$ & $\mathrm{DR}$ & $\begin{array}{l}\text { Age } \\
\text { (BP) }\end{array}$ \\
\hline Ly-2893 & $910-950$ & Pre-boreal & 0.7 & $9850+240$ \\
\hline-2894 & $\begin{array}{l}1060-1080 \\
1100-1120\end{array}$ & Late Dryas & 0.5 & $10,140 \pm 250$ \\
\hline-2895 & $1200-1230$ & Alleröd & 0.15 & $10,390 \pm 560$ \\
\hline
\end{tabular}


General Comment ( JLdeB): Ly-2893 is as expected. Ly-2894 comes from sec of boring which is too long, and Ly-2895 has too large a statistical margin; they look ca 500 and 1000 yr younger, respectively, compared to pollen data and unpub result from same boring at about same depths: 10251050cm: Gif-6216, 10,220 \pm 200; 1080-1100cm: Gif-6217, 10,600 \pm 200 ; and 1150-1180cm: Gif-6218, 11,200 \pm 150 (de Beaulieu, Pons, \& Reille, in press).

\section{Dar Fatma series, Aïn Draham, N W Tunisia}

Peat from six levels of boring in small bog at alt $910 \mathrm{~m}$ in La Kroumirie massif $\left(36^{\circ} 46^{\prime} \mathrm{N}, 8^{\circ} 42^{\prime} \mathrm{E}\right)$. Coll 1977 and subm 1980 by B Ben Tiba, SylvoPastoral Inst, Tunis and M Reille.

General Comment (MR): previous series confirmed that last deforestation was consequence of Arabian invasion: Ly-1650: $700 \pm 110$ (R, 1979, v 21, p 413) (Ben Tiba, 1980). Ly-2570 shows that first deforestation occurred at end of Atlantic. All other dates are much older than expected and show that boring reached Recent Pleistocene fms which had almost been eroded before Holocene fm of bog. Ly-2238, even with finite age, belongs to Pleistocene series and appears younger due to roots having penetrated from Holocene series (Ben Tiba \& Reille, 1982).

TABle 8

Dar Fatma

\begin{tabular}{cccc}
\hline $\begin{array}{c}\text { Sample } \\
\text { no. }\end{array}$ & $\begin{array}{c}\text { Depth } \\
(\mathrm{cm})\end{array}$ & DR & \multicolumn{1}{c}{$\begin{array}{c}\text { Age } \\
(\mathrm{BP})\end{array}$} \\
\hline Ly-2570 & $150-170$ & 0.05 & $4630 \pm 580$ \\
-2238 & $200-220$ & 0.5 & $19,640 \pm 530$ \\
-2569 & $225-250$ & 0.5 & $\geq=33,000$ \\
-2239 & $360-380$ & 1 & $\geq=28,200$ \\
-2241 & $380-400$ & 1 & $\geq=31,000$ \\
-2240 & $730-750$ & 0.8 & $\geq=33,700$ \\
\hline
\end{tabular}

\section{Pompillon series, Lans en Vercors, Isère}

Peat from ca $1.5 \mathrm{~m}$ depth, in middle of lacustrine clayey sediments $\left(45^{\circ} 6^{\prime} \mathrm{N}, 5^{\circ} 28^{\prime} \mathrm{E}\right)$. Coll and subm 1977 by G Monjuvent, Lab Geol, Univ Grenoble.

\section{Ly-1658. Pompillon 6}

$$
\begin{array}{r}
\geq 31,440 \begin{array}{r}
+1890 \\
-1560
\end{array} \\
\geq 33,500+5200 \\
-3100
\end{array}
$$$$
\text { Ly-2052. Pompillon 6b }
$$

General Comment (GM): despite apparent contemporaneity, both dates are too young and must be considered as min. Pollen strongly suggests interglacial environment dating to Eemian or Rissian interglacial.

\section{Les Echets series, Miribel, Ain}

Clay with high organic content from exploratory borings (1, A, B, and F) and a wider core-boring $(G)$, all drilled in glacio-lacustrine clay, deepest 
TABLE 9

Les Echets

\begin{tabular}{|c|c|c|c|c|}
\hline $\begin{array}{c}\text { Sample } \\
\text { no. }\end{array}$ & Boring & $\underset{(\mathrm{m})}{\text { Depth }}$ & DR & $\begin{array}{l}\text { Age } \\
(\mathrm{BP})\end{array}$ \\
\hline $\begin{array}{r}\text { Ly-2763 } \\
-2764 \\
-1908 \\
-1909 \\
-1747\end{array}$ & $\begin{array}{l}1 \\
1 \\
\mathrm{~A} \\
\mathrm{~A} \\
\mathrm{~A}\end{array}$ & $\begin{array}{c}3.9-4 \\
5.5-5.6 \\
\text { ca } 6.5 \\
\text { ca } 14.5 \\
21.8-21.9\end{array}$ & $\begin{array}{c}0.2 \\
0.3 \\
1 \\
1 \\
0.3\end{array}$ & $\begin{array}{l}11,910 \pm 350 \\
12,190 \pm 290 \\
16,250 \pm 480 \\
18,560 \pm 580 \\
24,300 \pm 1100\end{array}$ \\
\hline-1910 & B & ca 9.1 & 1 & $\begin{array}{r}32,100+1100 \\
-2300\end{array}$ \\
\hline $\begin{array}{l}-1746 \\
-2061 \\
-2765 \\
-2063 \\
-2766 \\
-2767 \\
-3060 \\
-2062 \\
-2768 \\
-2221 \\
-2769 \\
-2770 \\
-2771 \\
-3061 \\
-2772 \\
-3062\end{array}$ & $\begin{array}{l}B \\
F \\
G \\
G \\
G \\
G \\
G \\
G \\
G \\
G \\
G \\
G \\
G \\
G \\
G \\
G\end{array}$ & $\begin{array}{c}17-18 \\
24.5-24.6 \\
1.2-1.3 \\
2.9-3 \\
3.6-3.7 \\
4.05-4.15 \\
6.9-7.1 \\
11.7-11.8 \\
15.2-15.5 \\
20.2-20.3 \\
21.1-21.2 \\
22.1-22.2 \\
23-23.2 \\
23.7-23.8 \\
24.1-24.3 \\
25.5-25.6\end{array}$ & $\begin{array}{c}1 \\
1 \\
0.5 \\
1 \\
1 \\
0.5 \\
0.5 \\
1 \\
0.7 \\
0.7 \\
1 \\
0.8 \\
0.5 \\
0.4 \\
1 \\
0.8\end{array}$ & $\begin{array}{c}\geq 35,000 \\
\geq 34,000 \\
10,810 \pm 230 \\
15,050 \pm 250 \\
17,320 \pm 250 \\
15,260 \pm 290 \\
18,590 \pm 410 \\
17,530 \pm 270 \\
18,030 \pm 250 \\
20,050 \pm 380 \\
20,420 \pm 380 \\
21,120 \pm 400 \\
25,450 \pm 550 \\
21,760 \pm 650 \\
24,500 \pm 500 \\
\geq 33,700\end{array}$ \\
\hline
\end{tabular}

sediments in a large bog $\left(45^{\circ} 53^{\prime} \mathrm{N}, 4^{\circ} 55 \mathrm{E}\right)$. Ancient lake was inside innermost fold of external moraines of fluvio-glacial and glacial Würmian complex in Lyon region (Mandier, 1981). Coll from 1976 to 1979 and subm 1976 to 1983 by P Mandier, CNRS Assoc Lab No.260 and Lab Geog, Univ Lyon II, by G Monjuvent, by J L de Beaulieu and J Evin (Beaulieu et al, 1980).

General Comment (JLdeB \& PM): eight preliminary results helped to determine best location for main core boring $G, 56.6 \mathrm{~m}$ deep, which sampled sediments from Late Rissian to Late Würmian, as demonstrated by palynol study of all levels (Beaulieu \& Reille, 1984) and by ${ }^{14} \mathrm{C}$ dates of upper levels. Most dates for core $\mathrm{G}$ are in good strat sequence (fig 1 ) and suggest average sediment accumulation rate of ca $3 \mathrm{~m}$ per millennium below $6 \mathrm{~m}$ in middle part of lake during almost entire end of Late Würmian. Only Ly-2767, -3060 , and -2771 are out of sequence. Infinite ages obtained from below $24.5 \mathrm{~m}$ in boring $\mathrm{G}$ and neighboring boring $\mathrm{F}$, and at base of boring $\mathrm{B}$ (Ly-1746, -2062, -3062) confirm pollen data that suggest markedly slower sediment accumulation rate and even hiatus just before 25,000 BP. Pollen results also suggest that $24.5 \mathrm{~m}$ to $29 \mathrm{~m}$ dates to Würmian II, and $29 \mathrm{~m}$ to $34.5 \mathrm{~m}$, to Würmian I. Entire pollen diagram has been compared to that of La Grande Pile peat bog, NE France, previously studied by Woillard (1975) and dated by Groningen Lab.

\section{Romandie series, Switzerland}

Samples from palustrine and lacustrine sediments underlying moraines in Vaud and Fribourg dist. Coll 1979 to 1981 and subm 1980 and 1982 by R Arn, Lab Geol, Univ Lausanne. 


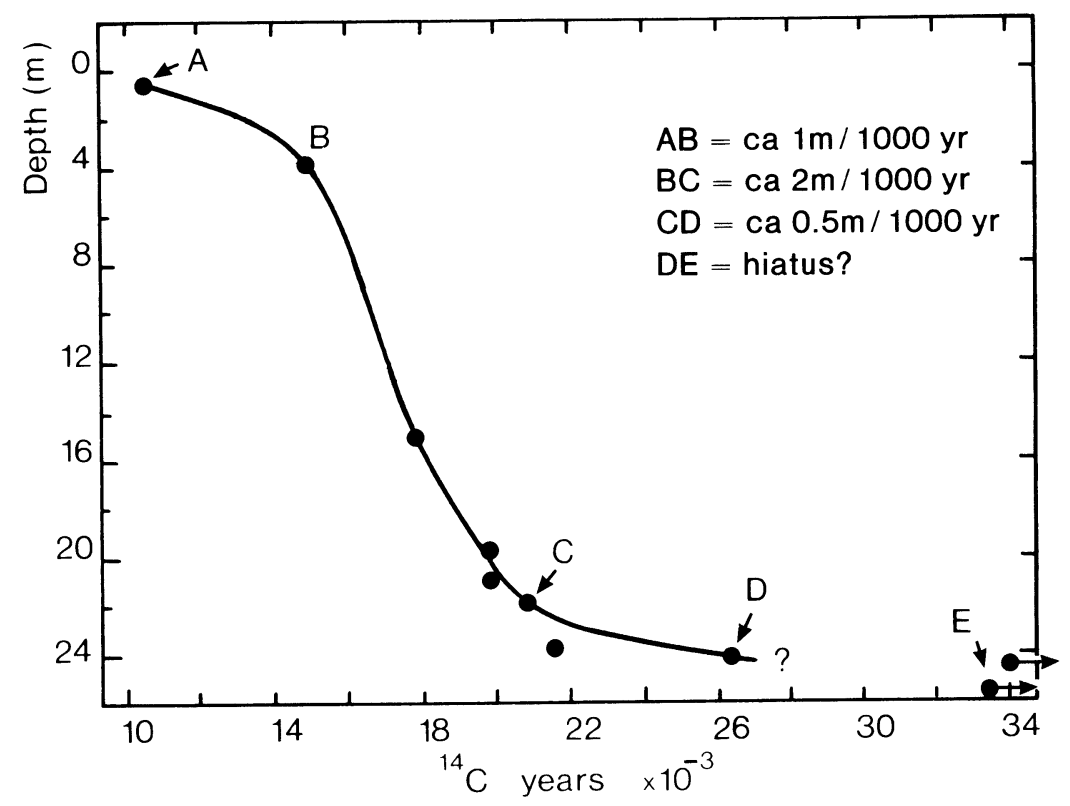

Fig 1. Sediment accumulation rate in boring $\mathrm{G}$ of Les Echets site

General Comment (RA): Ly-2580 fits previous measurement:

Ly-751: 34,600 ${ }_{-1800}^{+2700}$ from mammoth tusk in lower level of same site (R, 1975 , v 17, p 12). The five other results agree with pollen analyses. Peat from Pont-la-Ville contains temperate forest flora attributed to Early Würmian while other sites contain flora of cold climate with many herbaceous plants, and may be attributed either to Middle Würmian or Early Würmian interstadial, Signal de Bougy particularly (Arn, 1981).

TABLE 10

Romandie

\begin{tabular}{rlccc}
\hline $\begin{array}{c}\text { Sample } \\
\text { no. }\end{array}$ & \multicolumn{1}{c}{ Site } & Geog coordinates & Sample & Age (BP) \\
\hline Ly-2059 & Signal de Bougy, Vaud & $\left(46^{\circ} 29^{\prime} \mathrm{N}, 6^{\circ} 25^{\prime} \mathrm{E}\right)$ & Peaty slime & $\geq 32,000$ \\
-2060 & Tartegnin, Vaud & $\left(46^{\circ} 28^{\prime} \mathrm{N}, 6^{\circ} 9^{\prime} \mathrm{E}\right)$ & Peaty slime & $\geq 34,000$ \\
-2215 & Pont-la-Ville, Vaud & $\left(46^{\circ} 43^{\prime} \mathrm{N}, 6^{\circ} 06^{\prime} \mathrm{E}\right)$ & Wood & $\geq 36,000$ \\
-2216 & Saumont-Derrière, & $\left(46^{\circ} 31^{\prime} \mathrm{N}, 6^{\circ} 55^{\prime} \mathrm{E}\right)$ & Wood & $\geq 36,000$ \\
& Fribourg & $\left(46^{\circ} 36^{\prime} \mathrm{N}, 6^{\circ} 29^{\prime} \mathrm{E}\right)$ & Peat & $\geq 36,000$ \\
-2350 & Senarclans, Vaud & $\left(46^{\circ} 37^{\prime} \mathrm{N}, 6^{\circ} 35^{\prime} \mathrm{E}\right)$ & Peat & $25,090 \pm 550$ \\
-2580 & Bettens, Vaud & &
\end{tabular}

Samples from Caves

\section{W Pyrénées series, Hautes pyrénées and Pyrénées Atlantique}

Bones of large mammals from fill of several caves or pits. Subm 1982 by A Clot, Bordère sur Echez, as part of study on extinction of large mammals in Pyrénées massif at end of Würmian period. 
TABLE 11

West Pyrénées mammals

\begin{tabular}{|c|c|c|c|c|c|c|}
\hline $\begin{array}{c}\text { Sample } \\
\text { no. }\end{array}$ & Site, village, and dept & Geog coordinates & $\begin{array}{l}\text { Collector and } \\
\text { colln date }\end{array}$ & Fauna & DR & $\begin{array}{l}\text { Age }(\mathrm{BP}) \text { and } \\
\text { corrected } \\
\text { date interval } \\
(\mathrm{BC} / \mathrm{AD})\end{array}$ \\
\hline Ly-2856 & $\begin{array}{l}\text { Grotte d'Oillascoa, } \\
\text { Saint-Michel, } \\
\text { Pyrénées-Atlantique }\end{array}$ & $\left(43^{\circ} 04^{\prime} \mathrm{N}, \mathrm{l}^{\circ} 14^{\prime} \mathrm{W}\right)$ & $\begin{array}{c}\text { Speleol group } \\
\text { of Bayonne, } \\
1976\end{array}$ & Ursus spelaeus & 1 & $18,720 \pm 350$ \\
\hline-2857 & $\begin{array}{l}\text { Grotte de l'Oeil du Nez, } \\
\text { Rébénacq, } \\
\text { Pyrénées-Atlantique }\end{array}$ & $\left(43^{\circ} 09^{\prime} \mathrm{N}, 0^{\circ} 03^{\prime} \mathrm{W}\right)$ & $\begin{array}{l}\text { P Robert, } \\
1978\end{array}$ & Ursus spelaeus & 1 & $26,000 \pm 500$ \\
\hline-2858 & $\begin{array}{l}\text { Grotte de Couraü, } \\
\text { Saint-Pé de Bigorre, } \\
\text { Hautes Pyrénees }\end{array}$ & $\left(43^{\circ} 06^{\prime} \mathrm{N}, 0^{\circ} 09^{\prime} \mathrm{W}\right)$ & $\begin{array}{l}\text { P Robert, } \\
1947\end{array}$ & Ursus spelaeus & 0.9 & $28,870 \pm 700$ \\
\hline-2859 & $\begin{array}{l}\text { Grotte de Couret, } \\
\text { Ilhet, Hautes-Pyrénées }\end{array}$ & $\left(42^{\circ} 57^{\prime} \mathrm{N}, 0^{\circ} 06^{\prime} \mathrm{E}\right)$ & $\begin{array}{l}\text { A Clot, } \\
1967\end{array}$ & Cerous elephas & 1 & $\begin{array}{c}4230 \pm 160 \\
(3350-2425 \mathrm{BC})\end{array}$ \\
\hline-2860 & $\begin{array}{l}\text { Grotte de Peyreignes, } \\
\text { Tibiran-Jaunac, } \\
\text { Hautes-Pyrénées }\end{array}$ & $\left(43^{\circ} 03^{\prime} \mathrm{N}, 0^{\circ} 33^{\prime} \mathrm{E}\right)$ & $\begin{array}{l}\text { V Ferrer, } \\
1970\end{array}$ & Bison priscus & 1 & $14,470 \pm 230$ \\
\hline-2762 & $\begin{array}{l}\text { Grotte de Permayou, } \\
\text { Accous, } \\
\text { Pyrénées-Atlantique }\end{array}$ & $\left(42^{\circ} 56^{\prime} \mathrm{N}, 0^{\circ} 31^{\prime} \mathrm{W}\right)$ & $\begin{array}{l}\text { JP Besson \& } \\
\text { R Cabille, } \\
1972\end{array}$ & Capra pyrenaica & 1 & $\begin{array}{r}1520 \pm 120 \\
(\operatorname{AD} 240-690)\end{array}$ \\
\hline-2760 & $\begin{array}{l}\text { Gouffre PT 10, } \\
\text { Osse en Aspe, } \\
\text { Pyrénées Atlantique }\end{array}$ & $\left(43^{\circ} 00^{\prime} \mathrm{N}, 0^{\circ} 42^{\prime} \mathrm{W}\right)$ & $\begin{array}{c}\text { M Froissardet \& } \\
\text { H Laborde, } \\
1978\end{array}$ & Capra pyrenaica & 1 & $\begin{array}{c}5260 \pm 120 \\
(4405-3790 \mathrm{BC})\end{array}$ \\
\hline-2761 & $\begin{array}{l}\text { Grotte des Arrats, } \\
\text { Saint-Pé de Bigorre, } \\
\text { Hautes Pyrénées }\end{array}$ & $\left(43^{\circ} 04^{\prime} \mathrm{N}, 0^{\circ} 06^{\prime} \mathrm{W}\right)$ & $\begin{array}{l}\text { A Clot, } \\
1971\end{array}$ & Capra pyrenaica & 0.5 & $11,630 \pm 280$ \\
\hline
\end{tabular}

General Comment (AC): three dates on Ursus spelaeus bones confirm that extinction of cave bear was more recent than previously thought, as Ly2856 dates to end of Würmian III period. Ly-2860 suggests contemporaneity of dated fauna with Magdalenian settlement in neighboring Tiberan painted cave. Three dates on Capra pyrenaica show Pyrenean goat was present during entire Holocene (Clot \& Evin, 1983).

\section{Anthracology series, W Spain}

Charcoal from three levels in fills of two caves; subm by JL Vernet, Lab Bot, Univ Montpellier as part of anthracol study.

\section{Ly-2848. Cova Recambra, IVb}

$3850+160$

*2775-1930BC

From Layer IVb, in cave near Gandia, Valencia prov $\left(39^{\circ} 0^{\prime} \mathrm{N}, 0^{\circ} 20^{\prime}\right.$ W). Coll 1982 by J Apariocio-Perez (0.5 dr).

\section{Ly-2849. Cova Recambra, VII}

$5790 \pm 220$

*5000-4020BC $(0.2 \mathrm{dr})$.

From Layer VII, in same cave as Ly-2848. Coll 1962 by E Grau-Almero

\section{Ly-2850. Cova Ampla, II}

$6550 \pm 180$

From Layer II, in cave near Alicante, Alicante prov $\left(38^{\circ}, 23^{\prime} \mathrm{N}, 0^{\circ} 30^{\prime}\right.$ W). Coll 1982 by E Barlal-Garcia.

General Comment (JLV): dates confirm data from anthracol analyses that indicate first human deforestation is relatively old, while Ly-2848 appears a little too young from archaeol data. 
Ly-2313. Grotte de Bouxès, La Roque-Sainte-Marguerite, $9330 \pm 200$ Aveyron

Bones from clayey fill of cave $\left(44^{\circ} 8^{\prime} \mathrm{N}, 3^{\circ} 13^{\prime} \mathrm{E}\right)$. Coll and subm 1981 by A Tavoso, Prehist Dept, Univ Marseille. Comment (AT): date is younger than expected because assoc cold-climate fauna suggests more Tardiglacial period than Pre-boreal age.

\section{Ly-2614. Grotte du Bois du Cantet, Espèche, $\quad 10,920 \pm 160$} Hautes-Pyrénées

Bird bones from cave gallery ( $\left.43^{\circ} 3^{\prime} \mathrm{N}, 0^{\circ} 8^{\prime} \mathrm{E}\right)$. Coll 1977 by A Clot, subm by A Clot and C Mourer-Chauviré, Geol Lab, Univ Lyon I. C MourerChauviré attributes cave fill to Tardiglacial period by bird bones study. Comment (CM): date agrees with two previous measurements, Ly-1403: $13,370 \pm 270$ and Ly-1404: 13,060 \pm 430 from two other areas of same cave $(\mathrm{R}, 1979$, v 21, p 444) (Clot, 1934).

Ly-3001. Gouffre de Taille-Petit, Sainte-Orse, Dordogne $10,870 \pm 330$

Bones from fill of small pit $\left(45^{\circ} 22^{\prime} \mathrm{N}, 1^{\circ} 4^{\prime} \mathrm{E}\right)$. Coll 1982 by C MourerChauviré and subm by M Philippe, Mus Hist Natl, Lyon. Comment (MP): date is youngest of all samples from caves and pits of karstic calcareous plateaus, called "Causses" in region (see Gramat and Martel Causse series, R, 1979, v 21, p 416-418) (Philippe, Mourer-Chauviré, \& Evin, 1980).

\section{Igue de Barrière series, Miers, Lot}

Bones from fill in karstic network (44 $\left.53^{\prime} \mathrm{N}, 1^{\circ} 42^{\prime} \mathrm{E}\right)$. Coll 1981 and subm 1983 by $M$ Philippe. Provenience of previously dated bone sample, Ly-1576: 19,940 $\pm 800(\mathrm{R}, 1979, \mathrm{v} 21, \mathrm{p} 417)$ seems doubtful and is probably result of mixing of bone fragments from three other loci in site.

Ly-3030. Igue de Barrière No. 3

$19,340 \pm 1850$

From calcified dome at base of Pit $1(0.1 \mathrm{dr})$.

Ly-3031. Igue de Barrière No. 1

$24,420 \pm 560$

From same area as Ly-3030 (0.7 dr).

Ly-3002. Igue de Barrière No. 4

$27,290 \pm 710$

From partially emptied ancient gallery.

Ly-3003. Igue de Barrière No. 5

$1070 \pm 120$

$*_{A D} 770-1190$

From recent gallery which is deepest.

General Comment (MP): large differences in dates and collagen contents indicate that fill of karstic network comprises very complex mix of numerous successive layers of deposits, removals, and redepositions sometimes affecting only oldest sediments, in downward direction (Philippe \& Durand, 1984). 
Ly-2811. Grotte de la Balme Rousse, Choranche, Isère $\mathbf{2 6 , 0 0 0} \pm \mathbf{1 5 0 0}$

Bones (Ursus spelaeus) from Layer E3 at base of cave fill $\left(45^{\circ} 4^{\prime} \mathrm{N}, 50^{\circ}\right.$ $24^{\prime}$ E). Coll and subm 1982 by P Bintz, Lab Geol, Univ Grenoble ( $\left.0.2 \mathrm{dr}\right)$. Comment (PB): date corresponds to Würmian III and confirms that Ursus spelaeus became extinct in Alpine massif as late as in Pyrénées massif (see West Pyrénées mammals series, above).

\section{Jaurens series, Nespouls, Corrèze}

Bones from each extremity of fossiliferous layer of cave fill $\left(45^{\circ} 2^{\prime} \mathrm{N}, 1^{\circ}\right.$ 41' E). Coll 1970 and subm 1978 by C Mourer-Chauviré.

\section{Ly-1939. Jaurens, Sec 1}

$$
29,700+1500
$$

From sec 1 .

Ly-1938. Jaurens, Sec 20

$$
\begin{array}{r}
32,630 \\
-2100
\end{array}
$$

From sec 20.

General Comment (CM): dates are close and agree with two previous results from same site, Ly-359: 29,300 \pm 1400 and Ly-892:30,350 ${ }_{-1900}^{+3000}(\mathrm{R}$, 1976 , v 18, p 66-67). All four values indicate cave fill is homogeneous and dates to beginning of Würmian III (Guérin, Philippe, \& Vilain, 1979).

\section{Ly-2697. Grotte des Camisards, Sumène, Gard}

Bones (Ursus spelaeus) from top of fill of cave gallery $\left(43^{\circ} 58^{\prime} \mathrm{N}, 3^{\circ} 3^{\prime} \mathrm{E}\right)$. Coll and subm 1981 by A Bonnet, Nîmes. Comment (AB): date is older than others for Ursus spelaeus bones from caves in area, eg, Grotte Latrone cave, Ly-1966: 29,600 1100 (R, 1983, v 25, p 74) and La Baume Longue cave, Ly-2415: 26,500 $\pm 1000(\mathrm{R}, 1983$, v 25, p 73).

Samples from Fluvial and Fluvio-Glacial Sediments

\section{Ly-2527. Les Touches, Sinard, Isère}

$$
\delta^{14} \mathrm{C}=\mathbf{4 4 5} \begin{array}{r}
\text { Modern } \\
\pm 25 \%
\end{array}
$$

Stalks of Equisetum sp from glacio-lacustrine clay deposit corresponding to max advance of Würmian glacier in Le Triève region near Grenoble $\left(44^{\circ} 56^{\prime} \mathrm{N}, 5^{\circ} 40^{\circ} \mathrm{E}\right)$. Coll and subm 1981 by $\mathrm{G}$ Monjuvent, Lab Geol, Univ Grenoble. Comment (GM): date shows that despite ancient appearance and depth of burial in sediments, Equisetum could have grown quickly in rather compact clays.

\section{La Plaine series, Brégnier-Cordon, Ain}

Wood from several levels of Rhône R sediments $\left(45^{\circ} 39^{\prime} \mathrm{N}, 5^{\circ} 36^{\prime} \mathrm{E}\right)$. Coll and subm 1982 by J Evin and J P Bravard, Lab Geog, Univ Lyon III and 1983 (Ly-3051) by G Monjuvent. 
Ly-3051. Brégnier-Cordon No. 4

Wood from underlying moraine, coll by boring $(0.8 \mathrm{dr})$.

Ly-2777. Brégnier-Cordon No. 1

$1050 \pm 120$

Wood from fluviatile gravels.

Ly-2776. Brégnier-Cordon No. 2

$1420 \pm 140$

$*_{A D} 340-880$ gravels.

Wood from $\mathrm{S}$ sec of site, overlying clays that underlay fluviatile

Ly-2775. Brégnier-Cordon No. 3

$1660 \pm 110$

$*_{A D} 70-585$

Wood from same level as Ly-2776, in $\mathrm{N}$ sec of site $(0.7 \mathrm{dr})$.

General Comment (JPB): Ly-3051 shows this wood does not belong to geol fm. Ly-2776 and -2775 represent end of lacustrine sedimentation in valley which just precedes filling by gravel horizon, $10 \mathrm{~m}$ deep deposited after sudden change in river course, historically dated to Early Middle ages (Bravard, 1983) agrees perfectly with Ly-2777.

\section{Ly-3029. Pont de l'Hers, L'Union, Haute-Garonne}

$2770 \pm 120$

*1235-785BC

Fragment of oak trunk, buried ca $4 \mathrm{~m}$ deep in Hers $\mathrm{R}$ alluvium $\left(43^{\circ} 39^{\prime} \mathrm{N}, 1^{\circ} 28^{\prime} \mathrm{E}\right)$. Coll 1979 and subm 1983 by J C Revel, Lab Pédol, Univ Toulouse ( $0.8 \mathrm{dr})$. Assumed to be assoc with overlying Bronze age site (Gif-5499: $3660 \pm 100$, unpub). Comment (JCR): date is much younger than archaeol site and shows that river channel filling is complex, as only few $\mathrm{m}$ away, lowest level of fill is much more recent.

\section{Ly-1853. Les Crés, Saint-Maurice l'Exil, Isère}

$4770 \pm 180$

Wood from tree trunk found $3 \mathrm{~m}$ deep in alluvium of small tributary of Rhône R, near Nuclear Power Plant at Saint-Alban-Saint-Maurice $\left(45^{\circ} 31^{\prime} \mathrm{N}, 4^{\circ} 45^{\prime} \mathrm{E}\right)$. Coll and subm 1979 by M Délétie, Electricité France Soc, Paris. Comment (MD): date indicates Atlantic age for end of alluvial filling in tributary valley.

\section{Livet series, Isère}

Sample from La Romanche $\mathrm{R}$ alluvium in its middle valley $\left(45^{\circ} 6^{\prime} \mathrm{N}\right.$, $\left.5^{\circ} 56^{\prime} \mathrm{E}\right)$; subm 1982 by M Dubie, Electricité France Soc, Chambery.

Ly-2648. Tranchée de Livet

$590 \pm 120$

Charcoal from trench coll 1982. 
Ly-2616. Sondage du Pont de Livet

General Comment (MD): dates represent two stages of postglacial alluviation of valley. Ly-2616 indicates that large stone blocks found very deep in level may come from relatively recent collapse.

\section{La Haute-Romanche series, Hautes-Alpes}

Sample from two borings in high alt sediments drilled by hydroelectric equipment in high valley of Romanche R. Coll 1981 by Bachy Soc and subm 1982 by F Rosset, Electricité France Soc, Chambéry.

\section{Ly-2854. Plan de l'Alpe, Villar d'Arène}

$4870 \pm 130$

Wood, $9 \mathrm{~m}$ depth, in Boring VPA-8201 at alt $1960 \mathrm{~m}\left(45^{\circ} 1^{\prime} \mathrm{N}, 6^{\circ} 22^{\prime}\right.$ E).

\section{Ly-2853. Lac de Goléon, La Grave}

$5020 \pm 140$ E).

Peat, $3.5 \mathrm{~m}$ depth, in Boring VGO-8104 at alt $2440 \mathrm{~m}\left(45^{\circ} 5^{\prime} \mathrm{N}, 6^{\circ} 19^{\prime}\right.$

General Comment (FR): dates are in expected range, and represent end of Atlantic climatic phase. They prove that forest developed at much higher alt than present in this area, as well as in Alpe d'Huez area (above).

\section{Les Isles series, Champagneux, Savoie}

Sample from top and base of sandy formation underlying fluviatile gravels in stream channel of Rhône $\mathrm{R}\left(45^{\circ} 38^{\prime} \mathrm{N}, 5^{\circ} 39^{\prime} \mathrm{E}\right)$. Coll and subm 1982 by $\mathrm{J} P$ Bravard, Lab Geog, Univ Lyon III.

\section{Ly-2778. Les Isles de Champagneux, Tourbe}

$6330 \pm 140$ gravels.

Ly-2779. Les Isles de Champagneux, Bois

$8560 \pm 190$

Fragment of tree trunk at $12 \mathrm{~m}$ depth directly overlying glaciolacustrian sediments.

General Comment (JPB): Ly-2779 marks end of lacustrian filling of glacial valley of Rhône R; Ly-2778 marks beginning of fluviatile sedimentation.

\section{Ly-3059. Saint-Marceau, Orléans, Loiret}

$7300 \pm 140$

Wood from tree trunk at $6 \mathrm{~m}$ depth from boring in alluvium of Loire $\mathrm{R}$ $\left(47^{\circ} 54^{\prime} \mathrm{N}, 1^{\circ} 52^{\prime} \mathrm{E}\right)$. Coll and subm 1983 by Y M Allain, Service Espace Verts, Orléans. Comment (YMA): date indicates that alluvial substratum of area of Orléans, built several centuries ago, was deposited at beginning of Atlantic period. 


\section{Ly-3026. Moulin Tampon, Perreux, Loire}

$8430 \pm 130$

Wood from central part of tree trunk found at ca $2.5 \mathrm{~m}$ depth in alluvial plain of Loire $\mathrm{R}\left(46^{\circ} 2^{\prime} \mathrm{N}, 4^{\circ} 5^{\prime} \mathrm{E}\right)$. Coll 1981 and subm 1983 by $\mathrm{M}$ Vaginay, Dir Antiquités Hist Lyon. Comment (MV): date is of Boreal period, indicating relatively thick sedimentation in middle Loire valley since beginning of Holocene.

Ly-2981. Saint-Egrève, Isère

$10,310 \pm 170$

Wood, ca $20 \mathrm{~m}$ depth, in Isère R alluvia $\left(45^{\circ} 15^{\prime} \mathrm{N}, 5^{\circ} 41^{\prime} \mathrm{E}\right)$. Coll and subm 1983 by M Guimard, Electricité France Soc, Chambéry. Comment (MG): date indicates relatively thick alluviation in this valley from beginning of Holocene, coinciding with previous dates on this site, from similar samples, Sa-221: $7300 \pm 350$ and Sa-220: $9500 \pm 400(\mathrm{R}, 1975, \mathrm{v} 7, \mathrm{p} 239)$

\section{Ly-2815. Allaman, Vaud, Switzerland}

$13,090 \pm 160$

Bones (Bos primigenius) from terrace of Lake Leman at alt $400 \mathrm{~m}$ $\left(46^{\circ} 28^{\prime} \mathrm{N}, 6^{\circ} 21^{\prime} \mathrm{E}\right)$. Coll and subm 1982 by M Weidmann, Geol Mus, Lausanne. Comment (MW): date suggests Bölling period and confirms presence of this climatic phase in region (Arn, in press a).

\section{Pugneux series, Bressoles, Ain}

Vegetal debris from several levels of two sandy clay fms $\left(45^{\circ} 51^{\prime} \mathrm{N}\right.$, $5^{\circ} 6^{\prime} \mathrm{E}$ ). Coll and subm 1981 by R Vilain, Lab Geol, Univ Lyon I and J Evin. Lowest $\mathrm{fm}$ is terminal moraine of last Würmian glacier and uppermost $\mathrm{fm}$ is lacustrine deposited behind morain dam.

General Comment (RV \& JE): Ly-2317 and -2994 confirm paleontol data from rodents and gasteropods, and paleobot data which attribute Miocene origin to vegetal debris redeposited by Würmian glacier in lower $\mathrm{fm}$. Other results are in perfect strat agreement, indicating beginning of Würmian III period as min age for last retreat of Würmian glacier.

TABLE 12

Pugneux

\begin{tabular}{clcc}
\hline $\begin{array}{c}\text { Sample } \\
\text { no. }\end{array}$ & \multicolumn{1}{c}{ Location in section } & DR & Age (BP) \\
\hline Ly-2997 & Middle of upper fm & 0.5 & $17,300 \pm 510$ \\
-2996 & 10cm above base of upper fm & 0.7 & $22,580 \pm 500$ \\
-2995 & 10cm above base of upper fm & 1 & $22,640 \pm 600$ \\
-2993 & Base of upper fm & 0.7 & $23,440 \pm 450$ \\
-2318 & Base of upper fm & 1 & $24,110 \pm 900$ \\
-2994 & Top of lower fm & 1 & $\geq 35,000$ \\
-2317 & Ca 10cm under top of lower fm & 1 & $\geq 35,000$ \\
\hline
\end{tabular}

\section{Ly-2525. Font du Renard, Bras d'Asse,} Alpes de Haute Provence

Fragment of charred tree trunk, ca $4 \mathrm{~m}$ depth, at base of brown paleosol in Asse R alluvia ( $43^{\circ} 55^{\prime} \mathrm{N}, 6^{\circ} 17^{\prime} \mathrm{E}$ ). Coll 1980 and subm 1981 by $\mathrm{M}$ Dubar, Centre Recherche Archeol, Valbonne. Comment (MD): as sample 
was assoc with flora and fauna of temperate climate, date, as expected, may correspond to Würmian II/III interstadial, and shows that overlying cryoclastic sediments were deposited at end of Late Würmian (Dubar, 1983).

Ly-2515. Montagnat, Ain

Wood, $5 \mathrm{~m}$ depth, from boring in La Vallière $\mathrm{R}$ alluvia $\left(46^{\circ} 4^{\prime} \mathrm{N}, 5^{\circ} 18^{\prime}\right.$ E). Coll 1981 by G Vicherd, Dir Antiquités Hist, Lyon and subm by R Vilain. Comment (RV): date is much older than expected as overlying sediments seemed to be of Holocene origin. Thus, pronounced erosion must have occurred during early Quaternary.

Ly-2530. Armoy, Haute-Savoie

$\geq 35,000$

Peat from alt $545 \mathrm{~m}$ in Drance R valley $\left(46^{\circ} 21^{\prime} \mathrm{N}, 6^{\circ} 31^{\prime} \mathrm{E}\right)$. Coll and subm 1981 by R Arn. Comment (RA): date agrees with palynol, which suggests older age for all underlying fms (Arn, in press b) previously attributed to Würmian (Brun, 1977). Other samples, Gif-491: $\geq 35,000$ and Gif-739: $\geq 35,000$ (R, 1969, v 11, p 331) come from neighboring site.

\section{Samples from Various Continental Sediments}

\section{La Vieille Citerne series, Bonnevaux, Doubs}

Charcoal from soil in dolina containing three levels rich in charcoal, separated by two levels of silts, $10 \mathrm{~cm}$ thick $\left(46^{\circ} 50^{\prime} \mathrm{N}, 6^{\circ} 11^{\prime} \mathrm{E}\right)$. Coll 1979 and subm 1980 by M Gaiffe, Lab Bot, Univ Besançon.

General Comment (MG): dates indicate filling rate of ca $30 \mathrm{~cm}$ per millennium for bottom of dolina by material from erosion of neighboring soils (Gaiffe, 1983).

TABLE 13

La Vieille Citerne

\begin{tabular}{cccccc}
\hline $\begin{array}{c}\text { Sample } \\
\text { no. }\end{array}$ & $\begin{array}{c}\text { Ref } \\
\text { no. }\end{array}$ & $\begin{array}{c}\text { Depth } \\
(\mathrm{cm})\end{array}$ & DR & Age (BP) & $\begin{array}{c}{ }^{14} \text { C activity or } \\
\text { corrected } \\
\text { date interval (AD) }\end{array}$ \\
\hline $\begin{array}{r}\text { Ly-2362 } \\
-2363\end{array}$ & 1 & 8 & 0.7 & Modern & $\delta^{14} \mathrm{C}=-3 \pm 18 \% 0$ \\
-2258 & 2 & $30-35$ & 1 & Modern & $\delta^{14} \mathrm{C}=+9 \pm 14 \%$ \\
$620-1280$
\end{tabular}

\section{Vallon du Po series, Tiébelé, Haute-Volta}

Black potsherds from 1 to $1.5 \mathrm{~m}$ depth from top of alluvium of small valley $\left(11^{\circ} 14^{\prime} \mathrm{N}, 1^{\circ} 5^{\prime} \mathrm{W}\right)$. Coll 1982 by $\mathrm{M}$ Mietton and subm 1982 by $\mathrm{M}$ Chardon, Lab Geog, Univ Grenoble (0.8 dr).

\section{Ly-2802. P B F 1}

\section{Ly-2803. P B F 3}

$\mathbf{9 3 0} \pm \mathbf{2 0 0}$

$*_{A D 605-1420}$

$840 \pm 150$

$*_{A D} 905-1345$

General Comment (MC): despite lack of data on origin of carbon contained in black potsherd (Evin, 1983), both dates agree and indicate alluvium is 
recent, and erosion rate has been greater than sedimentation rate since 17 th century.

\section{Ly-2862. Uré, Ile des Pins, New Caledonia}

$3470 \pm 210$

$* 2305-1410 B C$

Bone debris (Silviornis neocaledoniae) and other vertebrates from corale breccia filling fossiliferous well in raised reef barrier of island in Kameruna Bay $\left(22^{\circ} 40^{\prime} \mathrm{S}, 167^{\circ} 25^{\prime} \mathrm{E}\right)$. Coll 1980, extracted from breccia cement using formic acid leaching and subm 1983 by F Poplin, Mus Nat Hist Natl Paris. Comment (FP): date confirms hypothesis based on previous measurement of cement of breccia from same site, Ly-2105: 19,490 \pm 330 (R, 1983, v 25, p 8); breccia should correlate ancient coral elements with very recent bone remains. It also proves Silviomis and other assoc animal sp have only recently become extinct, probably at time of human arrival on island (Poplin, Mourer-Chauviré, \& Evin, 1983).

\section{Baringo Lake series, Kenya}

Clay with low organic content from cores in basal sediments of lake in Gregory rift $\left(0^{\circ} 36^{\prime} \mathrm{N}, 36^{\circ} 6^{\prime} \mathrm{E}\right)$. Coll 1976 and 1978 and subm 1979 by $\mathrm{J} \mathrm{J}$ Tiercelin, Lab Quaternary Geol, Univ Marseille.

Ly-1822. BAR 78-3, No. 1

$1740 \pm 500$

From Levels 1 to 7, 0 to $20 \mathrm{~cm}$ depth in BAR 78-3 Core $(0.1 \mathrm{dr})$.

Ly-1823. BAR 78-3, No. 2

$920 \pm 270$

From Levels 31 to 37,90 to $112 \mathrm{~cm}$ in BAR $78-3$ Core $(0.2 \mathrm{dr})$.

Ly-1850. 62002

$5330 \pm 500$

From 0 to $22 \mathrm{~cm}$ depth in 62002 Core $(0.2 \mathrm{dr})$.

Ly-1758. 36016/36020

$2670 \pm 570$

From 75 to $105 \mathrm{~cm}$ depth in 36016 and 36020 Cores $(0.1 \mathrm{dr})$.

General Comment (JJT): first three dates appear aberrant. Ly-1822 and -1823 have inverted values for top and base of same core; Ly-1850 seems much too old for upper part of core. This may be due to complex origin of organic component of sediment which may come in part from older soil horizons (Tiercelin et al, 1981). Ly-1758 is meaningless because of mixing of sediments from two cores with no strat relationship.

\section{Bogoria Lake series, Kenya}

Samples from base of bank sediments of lake in Gregory rift $\left(0^{\circ} 15^{\prime} \mathrm{N}\right.$, $36^{\circ} 3^{\prime}$ E). Coll 1977 by J J Tiercelin and R W Renaut, Queen Mary College, London, and subm 1979 by J J Tiercelin.

Ly-1820. BOG 77-83 No. 1

$3900 \pm 410$

Clay with low organic content from Levels 1 to 6,0 to $20 \mathrm{~cm}$ depth, from BOG 77-83 Core $(0.3 \mathrm{dr})$. 


\section{Ly-1821. BOG 77-83 No. 2}

$3500 \pm 390$

Clay with low organic content from Levels 17 to 22,48 to $64 \mathrm{~cm}$ depth, from BOG 77-83 Core $(0.2 \mathrm{dr})$.

\section{Ly-1819. BOG 77-94}

$6290 \pm 460$

Clay with low organic content from Levels 20 to 24,59 to $73 \mathrm{~cm}$ depth from 77-94 Core $(0.3 \mathrm{dr})$.

\section{Ly-1818. BOG 77-62}

$6310 \pm 440$

Clay with low organic content from Levels 28 to 32,80 to $95 \mathrm{~cm}$ depth from BOG 77-62 Core.

\section{Ly-1981. Stromatolithe}

$\mathbf{3 7 3 0} \pm \mathbf{1 8 0}$

Stromatolithic calcareous crust from W side of lake at alt corresponding to former high level of lake.

\section{Ly-1982. Shells}

$15,520 \pm 420$

Shells (Melanoïdes sp) from W side of lake corresponding to former high level of lake.

General Comment (JJT): Ly-1981 and -1982 agree with expected ages and coincide with other results from identical samples (Young \& Renaut, 1979; Tiercelin, 1981). Other measurements from organic clays give less reliable results: Ly-1820 and -1821 are in inverted order, although they come from top and base of same core, whereas Ly-1818 and -1819 give dates which appear much too old compared to levels from core.

\section{Le Grand Etang series, La Réunion Island}

Wood from two levels in S3 Boring $\left(18^{\circ} \mathrm{S}, 55^{\circ} \mathrm{E}\right)$. Coll 1980 and subm 1981 by P Délétie, Electricité France Soc, Paris.

\section{Ly-2368. Le Grand Etang A}

$5650 \pm 180$

$* 4955-3975 B C$

From $24 \mathrm{~m}$ depth above level of volcanic scoria $(0.8 \mathrm{dr})$.

\section{Ly-2369. Le Grand Etang B}

$5000 \pm 290$

$* 4955-3975 B C$

From $26.8 \mathrm{~m}$ depth under same volcanic scoria level as Ly-2368.

General Comment (PD): although both dates are very close, they are in inverted strat order. However, they date to ca $5200 \mathrm{BP}$ with volcanic scoria level occurring between them; this level corresponds to eccentric eruption of La Fournaise volcano which agrees with other dates (Bachelery, 1981).

\section{Ly-2809. Blirh, Caïda de Ksabi, Prov Missour, Morocco $8260 \pm 180$}

Clay with large amount of small charcoal fragments from lacustrine sediments from presently arid region $\left(32^{\circ} 51^{\circ} \mathrm{N}, 4^{\circ} 13^{\prime} \mathrm{W}\right)$. Coll 1982 by $\mathrm{D}$ Lefevre and subm 1982 by J P Raynal, Lab Quaternary Geol, Univ Bordeaux I. Comment (JPR \& DL): as expected, date corresponds to beginning 
of Holocene, relatively humid climatic period in N Africa. Date confirms another unpub date, SUA-2014: $9220 \pm 110$ taken from carbonaceous concretions at top of same geol level (Lefevre, 1985).

\section{Izimane series, Hassi Bel Gebbour, Ouargla Willaya, Algeria}

Samples from four levels of geol outcropping on slope of isolated mound (26 45 $\mathrm{N}, 6^{\circ} 54^{\prime} \mathrm{E}$ ). Coll and subm 1981 (paleosol) and 1984 (calcareous) by A Bonnet, Nîmes.

\section{Ly-3082. Calcaire du Sommet}

$4200 \pm 190$

Dolomitic limestone and gypsum from top of sec $(0.6 \mathrm{dr})$.

\section{Ly-2642. Sommet du Paléosol}

$6780 \pm 140$

Organic matter from upper part of black hydromorphic paleosol, middle part of sec.

\section{Ly-2643. Base du Paléosol}

$8060 \pm 140$

Organic matter from base of same paleosol of Ly-2642.

\section{Ly-3083. Calcaire de la Base}

$9810 \pm 250$

Dolomitic limestone and gypsum from base of sec $(0.5 \mathrm{dr})$.

General Comment (AB): Ly-3082 agrees with expected age and marks end of sedimentation and beginning of present arid period. Ly-2642 perfectly agrees with two other dates from another black paleosol in Tichodaïne region, $\mathrm{Ly}-407: 6870 \pm 150(\mathrm{R}, 1973, \mathrm{v} 15, \mathrm{p} 146)$ and Ly-2483: $6010 \pm$ $160(\mathrm{R}, 1983, \mathrm{v} 25, \mathrm{p} 110)$. These three values date max of swamp vegetation in region which is now entirely desert. Ly-2643 marks beginning of humid period and confirmed by Ly-3083, which is assumed to come from lateral calcareous level equivalent to base of paleolsol and which may seem older due to influence of surrounding Mesozoic limestone (Roubet \& Matheu, 1970).

Ly-3032. Amguid, Tamanrasset Willaya, Algeria

$8370 \pm 200$

Calcareous tufa from upper part of tufa level of ancient waterfall near Amguid ( $26^{\circ} 26^{\prime} \mathrm{N}, 5^{\circ} 23^{\prime} \mathrm{E}$ ). Coll 1982 and subm 1983 by A Bonnet. Comment $(\mathrm{AB})$ : as waterfall probably flowed for last time during last Saharan Pluvial, date agrees perfectly with expected age of this humid climatic phase, and corresponds to base of Izimane series (above) as well as to another measurement from lacustrine limestone near Hirafok, Gif-325: $8380 \pm 300$ (Delibrias \& Dutil, 1966).

\section{Oued series, N Tunisia}

Samples from alluvial sediments from valleys of three wadis. Coll 1977 (Ly-2897) and 1982 and subm 1982 by A Miossec, Lab Geog, Univ Nantes. 
Ly-2899. Oued Hamman, Argoub Hassine, Nefza Dept (AM): date shows charcoals do not belong to geol $\mathrm{fm}$.

Ly-2900. Oued El Hamman, Ragoubet Tassera, Nefza Dept

$5870 \pm 320$

*5305-4140BC

Gastropod shells from scree slope older than low terrace of wadi $\left(41^{\circ}\right.$ $\left.14^{\prime} \mathrm{N}, 7^{\circ} 54^{\prime} \mathrm{E}\right)(0.2 \mathrm{dr})$. Comment (AM): date shows relatively late Holocene deposit of scree, but difference from Ly-2897 (below) does not seem significant.

Ly-2897. Djebel El Hara, Hedil region, Joumine Dept $8690 \pm 280$

Small gastropod shells from slope deposit in small wadi valley $\left(41^{\circ} 1^{\prime} \mathrm{N}\right.$, $7^{\circ} 89^{\prime} \mathrm{E}$ ). Comment (AM): date agrees with expected age as it shows that slope deposit took place at beginning of Holocene before this deposit of "Rhabian" terrace which is older than series of cones from Nefza region (Ly-2900, above).

Ly-2581. Cours Sablon, Clermont-Ferrand, Puy-de-Dôme $\geq 32,000$

Sandy silts with high organic content from foundation of bldg in city $\left(45^{\circ} 47^{\prime} \mathrm{N}, 3^{\circ} 5^{\prime} \mathrm{E}\right)$. Coll and subm 1981 by J P Daugas, Dir Antiquités Prehist Clermont-Ferrand, and J P Raynal. Level underlies pyroclastic volcanic sediments from the Clermont maar (ancient lake in crater of extinct volcano). Comment (JPD \& JPR): as expected, result is infinite age because volcanic eruption surely occurred before Würmian period, as suggested by TL date by Clermont Lab: Cler-TL-23: 156,000 \pm 17,000 before 1980 (Miallier, 1982).

Samples from Marine and Lagoonal Sediments

\section{Ly-2896. Sondage DJ6, Ahémé lake, Bénin}

$\delta^{14} \mathrm{C}=+8 \pm 18 \%$

Shells from Level $6,30 \mathrm{~cm}$ depth, from boring in Aho R bank, between Lake Ahémé and sea $\left(6^{\circ} 19^{\prime} \mathrm{N}, 1^{\circ} 58^{\prime} \mathrm{E}\right)$. Coll 1982 by M Oyédé, Univ Cotonou, and subm 1983 by J Lang, Lab Geol, Univ Dijon (0.5 dr). Comment ( JL \& MO): slightly older date was expected. This date shows change in sedimentation rate in $\mathrm{N}$ part of Lake Ahémé (Oyédé, 1983).

\section{Bonthe Distric Coast series, Sierra Leone}

Shells from top of offshore sand bar in coastal sites. Coll 1979 and 1980 by E Anthony and subm 1981 by P Michel Lab Geol, Univ Strasbourg.

General Comment (EA): dates of ca 2000 BP were expected because of previous measurements made by Gif Lab (Labeyrie \& Delibrias, 1976). Dates show that samples come from kitchen middens of recent human settlements and have no relation to sea level change. 
TABLE 14

Bonthe District

\begin{tabular}{|c|c|c|c|c|c|c|}
\hline $\begin{array}{c}\text { Sample } \\
\text { no. }\end{array}$ & Site & Geog coordinates & Sample & DR & $\begin{array}{r}\text { Age } \\
(\mathrm{BP})\end{array}$ & $\begin{array}{l}\text { Corrected } \\
\text { date } \\
\text { interval or } \\
{ }^{14} \mathrm{C} \text { activity }\end{array}$ \\
\hline $\begin{array}{r}\text { Ly- } 2484 \\
-2485 \\
-2486\end{array}$ & $\begin{array}{l}\text { Yoni } \\
\text { Tisana } \\
\text { Jimaï }\end{array}$ & $\begin{array}{l}\left(7^{\circ} 29^{\prime} \mathrm{N}, 12^{\circ} 30^{\prime} \mathrm{W}\right) \\
\left(7^{\circ} 35^{\prime} \mathrm{N}, 12^{\circ} 37^{\prime} \mathrm{W}\right) \\
\left(7^{\circ} 25^{\prime} \mathrm{N}, 12^{\circ} 30^{\prime} \mathrm{W}\right)\end{array}$ & $\begin{array}{l}\text { Anadara senelis } \\
\text { Anadara senelis } \\
\text { Anadara senelis } \\
\text { Crasostrea gasar }\end{array}$ & $\begin{array}{l}0.7 \\
1 \\
0.8\end{array}$ & $\begin{array}{l}690 \pm 140 \\
\text { Modern } \\
\text { Modern }\end{array}$ & $\begin{array}{c}\text { AD } 1190-1480 \\
\delta^{14} \mathrm{C}=-15 \pm 18 \% \\
\delta^{14} \mathrm{C}=+29 \pm 19 \% 0\end{array}$ \\
\hline-2487 & Baki & $\left(7^{\circ} 35^{\prime} \mathrm{N}, 13^{\circ} 00^{\prime} \mathrm{W}\right)$ & Undetermined & 0.7 & Modern & $\delta^{14} \mathrm{C}=+1 \pm 19 \% 0$ \\
\hline
\end{tabular}

\section{Red Sea coast series, Sudan}

Samples from Marsa Odudu $\left(21^{\circ} 3^{\prime} \mathrm{N}, 37^{\circ} 3^{\prime} \mathrm{E}\right)$ and Aydhab $\left(22^{\circ} 20^{\prime} \mathrm{N}\right.$, $36^{\circ} 30^{\prime} \mathrm{E}$ ) on Red Sea coast. Coll and subm 1981 by R Dalongeville, Maison de l'Orient Méditérranéen, Univ Lyon II.

General Comment (RD): all results are very consistent with expected values (Dalongeville \& Sanlaville, 1981) but Ly-2466 must be considered according to widest statistical margin.

TABLE 15

Sudan Coast

\begin{tabular}{|c|c|c|c|c|c|c|c|}
\hline $\begin{array}{c}\text { Sample } \\
\text { no. }\end{array}$ & Site & $\begin{array}{l}\text { Ref } \\
\text { no. }\end{array}$ & Sample & $\mathrm{DR}$ & $\begin{array}{l}\text { Expected } \\
\text { age } \\
\text { (centuries) }\end{array}$ & $\begin{array}{c}\text { Age } \\
(\mathrm{BP})\end{array}$ & $\begin{array}{l}\text { Corrected date } \\
\text { interval or } \\
{ }^{14} \mathrm{C} \text { activity }\end{array}$ \\
\hline Ly-2465 & Marsa Odudu & 11 & Corals & 1 & recent & Modern & $\delta^{14} \mathrm{C}=+178 \pm 17 \%$ \\
\hline-2466 & Aydhab & 24 & Charcoal & 0.15 & 9 th-16th & Modern & $\delta^{14} \mathrm{C}=+25 \pm 25 \%$ \\
\hline-2467 & Aydhab & 25 & Shells & 1 & $9 \mathrm{th}-16 \mathrm{th}$ & $790 \pm 120$ & AD $1040-1335$ \\
\hline-2468 & Aydhab & 27 & Shells & 1 & 5 th- 10 th & $270 \pm 110$ & AD $1420-1950$ \\
\hline-2469 & Aydhab & 28 & Corals & 0.6 & Holocene & Modern & $\delta^{14} \mathrm{C}=+170 \pm 23 \%$ \\
\hline-2470 & Aydhab & 30 & Charcoal & 0.15 & 5 th- 15 th & $630 \pm 200$ & AD $1045-1610$ \\
\hline
\end{tabular}

\section{Coast series, La Grande-Terre Island, Guadeloupe}

Sample from continental sediments from boring at $-5.4 \mathrm{~m}$ under msl on $\mathrm{W}$ coast (Ly-2952) and from raised beaches at $+2 \mathrm{~m}$ and $+3 \mathrm{~m}$ on $\mathrm{E}$

TABLE 16

La Grande Terre Island

\begin{tabular}{|c|c|c|c|c|c|c|}
\hline $\begin{array}{l}\text { Sample } \\
\text { no. }\end{array}$ & Site & Geog coordinates & $\begin{array}{l}\text { Ref } \\
\text { no. }\end{array}$ & Sample & $\begin{array}{l}\text { Age } \\
\text { (BP) }\end{array}$ & $\begin{array}{l}\text { Corrected } \\
\text { date } \\
\text { interval }\end{array}$ \\
\hline Ly-2952 & $\begin{array}{l}\text { Belle Plaine, } \\
\text { Les Abymes }\end{array}$ & $\left(16^{\circ} 18^{\prime} \mathrm{N}, 61^{\circ} 31^{\prime} \mathrm{W}\right)$ & 0 & Peat & $4010 \pm 180$ & $3020-2120 \mathrm{BC}$ \\
\hline-2945 & $\begin{array}{l}\text { Pointe Gros Boeuf, } \\
\text { Ste Anne }\end{array}$ & $\left(16^{\circ} 14^{\prime} \mathrm{N}, 61^{\circ} 18^{\prime} \mathrm{W}\right)$ & 1 & Charcoal & $2090 \pm 120$ & 395BC-AD 195 \\
\hline-2947 & $\begin{array}{l}\text { Pointe Gros Boeuf, } \\
\text { Ste Anne }\end{array}$ & $\left(16^{\circ} 14^{\prime} \mathrm{N}, 61^{\circ} 18^{\prime} \mathrm{W}\right)$ & 3 & Coral & $2480 \pm 160$ & $865 \mathrm{BC}-260 \mathrm{BC}$ \\
\hline-2949 & $\begin{array}{l}\text { Pointe Gros Boeuf, } \\
\text { Ste Anne }\end{array}$ & $\left(16^{\circ} 14^{\prime} \mathrm{N}, 61^{\circ} 18^{\prime} \mathrm{W}\right)$ & 5 & Shells & $3920 \pm 200$ & $2905-1950 \mathrm{BC}$ \\
\hline-2950 & $\begin{array}{l}\text { Pointe Gros Boeuf, } \\
\text { Ste Anne }\end{array}$ & $\left(16^{\circ} 14^{\prime} \mathrm{N}, 61^{\circ} 18^{\prime} \mathrm{W}\right)$ & 6 & Beach rock & $2650 \pm 100$ & $1040-595 \mathrm{BC}$ \\
\hline-2946 & $\begin{array}{l}\text { Plage Gros Boeuf, } \\
\text { St François }\end{array}$ & $\left(16^{\circ} 14^{\prime} \mathrm{N}, 61^{\circ} 17^{\prime} \mathrm{W}\right)$ & 2 & Coral & $3080 \pm 160$ & $1685-910 \mathrm{BC}$ \\
\hline-2948 & $\begin{array}{l}\text { Porte d'Enfer, } \\
\text { Le Moule }\end{array}$ & $\left(16^{\circ} 19^{\prime} \mathrm{N}, 61^{\circ} 18^{\prime} \mathrm{W}\right)$ & 4 & Shells & $1000 \pm 120$ & AD870-1230 \\
\hline-2953 & $\begin{array}{l}\text { Baie du Moule, } \\
\text { Le Moule }\end{array}$ & $\left(16^{\circ} 20^{\prime} \mathrm{N}, 61^{\circ} 21^{\prime} \mathrm{W}\right)$ & 9 & Coral & $27,600 \pm 650$ & \\
\hline
\end{tabular}


coast. Coll and subm 1982 by A Assor and A Klingebiel, Lab Geol, Univ Bordeaux.

General Comment (AK): dates confirm shifting of La Grande Terre I. due to westward movement downward and eastward rising along axis, La Plaine des Abymes-Grand Culs de Sac-Pointe du Château.

\section{Carribee Sea coast series, Colombia}

Sample from marine sediments outcropping several $m$ above present msl in Cartagen Bay. Coll 1979 and subm 1981 by T Burel, Lab Geol, Univ Bordeaux I.

General Comment (TB): dates agree with two previous measurements, from Tierrabomba I. (unpub), $2850 \pm 150$ (Richards \& Broecker, 1963), and from Cienaga Honda, S part of Catagena Bay, Gif-5038: $2700 \pm 90$ (Burel, Klingebiel, \& Vernette, 1982). They confirm contemporaneity of deposits of shell conglomerates and building of coral reef during rise in sea level at ca 3000 BP.

TABLE 17

Carribee Sea coast

\begin{tabular}{|c|c|c|c|c|c|c|}
\hline $\begin{array}{c}\text { Sample } \\
\text { no. }\end{array}$ & Site & $\begin{array}{l}\text { Ref } \\
\text { no. }\end{array}$ & Geog coordinates & Sample & $\begin{array}{r}\text { Age } \\
(\mathrm{BP})\end{array}$ & $\begin{array}{c}\text { Corrected } \\
\text { date } \\
\text { interval }\end{array}$ \\
\hline Ly-2477 & Tierrabomba I. & 24 bis & $\left(10^{\circ} 24^{\prime} \mathrm{N}, 75^{\circ} 35^{\prime} \mathrm{W}\right)$ & She & 1930 & 165 \\
\hline-2479 & Tierrabomba I. & 30 & $\left(10^{\circ} 24^{\prime} \mathrm{N}, 75^{\circ} 33^{\prime} \mathrm{W}\right)$ & Coral & $2670 \pm 120$ & AD65-585 \\
\hline-2481 & Tierrabomba I. & 79TB21 & $\left(10^{\circ} 24^{\prime} \mathrm{N}, 75^{\circ} 35^{\prime} \mathrm{W}\right)$ & Coral & $3690 \pm 160$ & $2530-1735 \mathrm{BC}$ \\
\hline-2478 & Escuela Naval & 333 & $\left(10^{\circ} 25^{\prime} \mathrm{N}, 75^{\circ} 30^{\prime} \mathrm{W}\right)$ & Coral & $2470 \pm 120$ & $810-395 \mathrm{BC}$ \\
\hline-2480 & Manzanillo & 140 & $\left(10^{\circ} 31^{\prime} \mathrm{N}, 75^{\circ} 40^{\prime} \mathrm{W}\right)$ & Shell & $1450 \pm 130$ & AD $250-865$ \\
\hline-2482 & Tesoro I. & 131 & $\left(10^{\circ} 18^{\prime} \mathrm{N}, 75^{\circ} 40^{\prime} \mathrm{W}\right)$ & Coral & $1780 \pm 120$ & AD $15-440$ \\
\hline
\end{tabular}

\section{Ly-2633. Moulay Bou Salham, Kénitra Prov, Morocco}

$2170 \pm 210$ lagoon $\left(34^{\circ} 50^{\prime} \mathrm{N}, 6^{\circ} 13^{\prime} \mathrm{W}\right)$. Coll 1978 and subm 1982 by C Carruesco, Lab Geol, Univ Bordeaux I. Comment (CC): date agrees with Gif-4542: 3490 \pm 100 (unpub) from underlying level, 59 to $64 \mathrm{~cm}$ depth, of same site and marks end of Mellahian transgression, also dated in Oualidia lagoon (below) (Bidet \& Carruesco, 1980)

\section{Oualidia series, El Jalida Prov, Morocco}

Shell debris from borings in dessicating lagoon $\left(32^{\circ} 45^{\prime} \mathrm{N}, 9^{\circ} 0^{\prime} \mathrm{W}\right)$. Samples come from transgressional marine sediments underlying lagoon sediments. Coll 1977 and subm 1978 by C Carruesco.

TABLE 18

Oualidia

\begin{tabular}{cccccc}
\hline $\begin{array}{c}\text { Sample } \\
\text { no. }\end{array}$ & $\begin{array}{c}\text { Boring } \\
\text { no. }\end{array}$ & $\begin{array}{c}\text { Depth } \\
(\mathrm{cm})\end{array}$ & DR & $\begin{array}{c}\text { Age } \\
(\mathrm{BP})\end{array}$ & $\begin{array}{c}\text { Corrected } \\
\text { date } \\
\text { interval }\end{array}$ \\
\hline Ly-2627 & 77008 & $30-35$ & 0.07 & $4880 \pm 490$ & $4325-3035$ \\
-2628 & 77013 & $50-55$ & 1 & $2120 \pm 130$ & $415 \mathrm{BC}-\mathrm{AD} 210$ \\
-2629 & 77014 & $60-75$ & 1 & $3840 \pm 130$ & $1770-1940 \mathrm{BC}$ \\
-2630 & 77016 & $50-55$ & 1 & $3320 \pm 130$ & $1965-1360 \mathrm{BC}$ \\
-2631 & 77018 & $80-85$ & 0.25 & $3330 \pm 240$ & $2110-1145 \mathrm{BC}$ \\
-2632 & 77019 & base & 0.8 & $6420 \pm 190$ & $5915-4995 \mathrm{BC}$ \\
\hline
\end{tabular}


General Comment (CC): dates mark beginning and max of Mellahian transgression in region, also called Flandrian or Nouakchottian, often dated in Mauritania (Ortlieb, 1980).

\section{Ly-2615. Tin Ouéich, Mauritania}

$4520 \pm 130$

*3545-2900BC

Shells (Cerastoderma edule) from lagoonal clayey sand level $\left(18^{\circ} 4^{\prime} \mathrm{N}\right.$, $\left.15^{\circ} 49^{\prime} \mathrm{W}\right)$. Coll and subm 1982 by D Carité, Nouakchott. Sample was dated to confirm geol interpretation of site previously based on dates: Ly-2160: $35,000+1900$ and Ly-2189: 29,900 +600 (R, 1983, v 25, p 64). Comment (DC): date corresponding to max of Nouakchottian transgression shows that level dated to Inchirian was later cut by channel before Nouakchottian transgression, which deposited sediment with same facies as Inchirian (Carité, 1983).

\section{Sea coast series, Bahraïn}

Samples from two coastal archaeol sites, Ras Hayyan $\left(26^{\circ} 2^{\prime} \mathrm{N}, 50^{\circ} 38^{\prime}\right.$ E) and Qalaat el Bahraïn (26 $14^{\prime} \mathrm{N}, 50^{\circ} 31^{\prime} \mathrm{E}$ ). Coll and subm 1982 by $\mathrm{P}$ Sanlaville, Maison Orient Méditérranéen, Univ Lyon II, and R Paskoff, Univ Tunis, during study of sea level changes and human settlements on coast.

General Comment (PS): at Qalaat, except for Ly-2868, which was probably redeposited older material, all dates suggest evolution of sea shore occurred as expected from results of strat study of archaeol sites and agree with most Birmingham dates from same site (Doornkamp, Brunsden, \& Jones, 1980). At Ras Hayyen, Ly-2872 fits better with expected value than 4 Birmingham dates, ca 7000 to $6000 \mathrm{BP}$.

TABLE 19

Bahraïn Sea coast

\begin{tabular}{|c|c|c|c|c|c|}
\hline $\begin{array}{c}\text { Sample } \\
\text { no. }\end{array}$ & Site & Sample & $\begin{array}{c}\text { Alt } \\
(\mathrm{cm})\end{array}$ & $\begin{array}{c}\text { Age } \\
(\mathrm{BP})\end{array}$ & $\begin{array}{l}\text { Corrected } \\
\text { date } \\
\text { interval } \\
\text { (BC) }\end{array}$ \\
\hline Ly-2871 & Qalaat & Shells & -80 & $3430 \pm 120$ & $2030-1520$ \\
\hline-2870 & Qalaat & Shells & $+35-15$ & $3260 \pm 160$ & $1885-1260$ \\
\hline-2869 & Qalaat & Shells & $+65-35$ & $3030 \pm 120$ & $1550-915$ \\
\hline-2868 & Qalaat & Vermitidae crust & $+50-100$ & $5960 \pm 140$ & $5240-4450$ \\
\hline-2867 & Qalaat & Vermitidae crust & $+50-100$ & $4350 \pm 160$ & $3470-2650$ \\
\hline-2872 & Ras Hayyan & Shells & +200 & $5070 \pm 160$ & $4135-3640$ \\
\hline
\end{tabular}

\section{Sidi Salhem formation series, Gabès gulf, $\mathbf{S}$ Tunisia}

Mollusk shells from shelly sands in Sidi-Salhem sandstone dunes, in Jerba I. region. Coll and subm 1982 by R Paskoff and P Sanlaville. 
Ly-2617. Borj Gourine

Shells (Helix sp) from Gourine ancient Borj $\left(33^{\circ} 39^{\prime} \mathrm{N}, 10^{\circ} 56^{\prime} \mathrm{E}\right)$ $(0.7 \mathrm{dr})$.

\section{Ly-2618. Borj Gastil}

$6890 \pm 390$

*6550-5220BC

Shells (Helix sp, Lucina sp) from S coast of Jerba I. (33 $42^{\circ} \mathrm{N}, 10^{\circ} 56^{\prime}$ E) $(0.15 \mathrm{dr})$.

General Comment (PS): both dates attribute Holocene age to Sidi-Salhem sandstone, previously assumed to be marine deposit from Middle Würmian (Paskoff \& Sanlaville, 1983). They agree with other unpub results from Monaco lab.

\section{Ly-2873. Sidi Fredj, Kerkennah I., S Tunisia}

$17,850 \pm 430$

Shells (Helix sp) from red sandy silt outcropping on beach in Cherguia I. $\left(39^{\circ} 49^{\prime} \mathrm{N}, 11^{\circ} 8^{\prime} \mathrm{E}\right)$. Coll 1981 and subm 1983 by $\mathrm{P}$ Sanlaville and $\mathrm{R}$ Paskoff. Comment (PS): silts were attributed to Holocene because of presence of Neolithic potsherds. However, date shows they are redeposited Würmian silts. Other measurements showing importance of neotectonics in site were pub previously $(\mathrm{R}, 1983, \mathrm{v} 25, \mathrm{p} 83)$.

\section{Le Tlêt formation series, Gabès gulf, $S$ Tunisia}

Samples from Tlêt shelly sandstone outcropping forming slope deposit on sea shore. Coll and subm 1982 by R Paskoff and P Sanlaville.

Ly-2644. Oued Fahmine $20,690 \pm 360$

Shells of various sp from $\mathrm{S}$ shore of Jerba I. $\left(33^{\circ} 43^{\prime} \mathrm{N}, 10^{\circ} 51^{\prime} \mathrm{E}\right)$.

\section{Ly-2645. Guellala and Oued Ogla}

$23,090 \pm 560$

Small fragments of ostrich eggs from two sampling areas in Tlêt sandstone, near wadi Ogla $\mathrm{R}$ valley on Zarzis peninsula $\left(33^{\circ} 34^{\prime} \mathrm{N}, 10^{\circ} 55^{\prime} \mathrm{E}\right)$ $(0.5 \mathrm{dr})$.

General Comment (PS): agreement of these dates confirms that ostrich eggs may be used as dating material, even in old sediments (Evin, 1983). Although both dates seem ca 2 or 3 milliennia too young, they prove Tlêt $\mathrm{fm}$ is not Tyrrenian (ca 85,000 BP), but is rather Würmian, ca 27,000 BP in Gabès gulf region.

\section{Coastal Crete and Naxos I. series, Greece}

Samples from $S$ shore of Crete I. at Preveli near Ayla Galini $\left(35^{\circ} 8^{\prime} \mathrm{N}\right.$, $\left.24^{\circ} 40^{\prime} \mathrm{E}\right)$, at Akrotiri, near Khania ( $\left.35^{\circ} 8^{\prime} \mathrm{N}, 24^{\circ} 3^{\prime} \mathrm{E}\right)$, from $\mathrm{N}$ shore of Crete I. at Aghia Barbara, near Mallia ( $35^{\circ} 16^{\prime} \mathrm{N}, 25^{\circ} 30^{\prime} \mathrm{E}$ ), and from N shore of Naxos I. at Palati cave near Naxos ( $\left.37^{\circ} 6^{\prime} \mathrm{N}, 25^{\circ} 22^{\prime} \mathrm{E}\right)$. Coll and subm by R Dalongeville, during study of shoreline variation.

General Comment (RD): large age differences are due to variations in influence of neotectonics at different points along shoreline. Thus, Ly- 
TABLE 20

Crete and Naxos Islands

\begin{tabular}{|c|c|c|c|c|c|c|c|}
\hline $\begin{array}{c}\text { Sample } \\
\text { no. }\end{array}$ & Site & $\begin{array}{l}\text { Ref } \\
\text { no. }\end{array}$ & Sample & $\mathrm{DR}$ & Expected age & $\begin{array}{l}\text { Age } \\
\text { (BP) }\end{array}$ & $\begin{array}{c}\text { Corrected } \\
\text { date } \\
\text { interval }\end{array}$ \\
\hline Ly-2471 & Preveli & & Vermetidae & 1 & 5 th century $\mathrm{AD}$ & $2320 \pm 160$ & $785-45 \mathrm{BC}$ \\
\hline-2472 & Aghia Barbara & 1 & Vermetidae & 1 & ca $1600 \mathrm{BC}$ & $1420 \pm 120$ & AD435-785 \\
\hline-2473 & Aghia Barbara & 2 & Vermetidae & 0.8 & ca $1600 \mathrm{BC}$ & $22,840 \pm 450$ & \\
\hline-2474 & Aghia Barbara & 3 & Vermetidae & 0.8 & Holocene & $25,530 \pm 440$ & \\
\hline-2475 & Grotta Palati & 1 & Cardium shells & 1 & $2800 \mathrm{BC}$ & $5560 \pm 160$ & $4710-3960 \mathrm{BC}$ \\
\hline-2476 & Akrotiri & & Vermetidae & 1 & $3000 \mathrm{BC}$ & $11,570 \pm 170$ & \\
\hline
\end{tabular}

2473 and -2474 show some sediments were formed during transgression prior to Flandrian.

\section{N W Mediterranean Sea series}

Calcium carbonate from marine sediments from cores from Rhône $\mathrm{R}$ delta to W shore of Corsica. Coll 1981 by M Fernex, Lab Marine Geol, Villefranche-sur-Mer, and subm by L Blanc, Lab Quaternary Geol, Univ Marseille and MT Morzadec, Lab Geol, Univ Rennes. All measurements were made on total carbonate fraction even where detritic elements might have been present, as in previous study on Pelagian Sea (Burollet \& Winnock, 1979).

General Comment (LB \& MTM): for Cores KS3030, MKS7, and KSPF, there seems to be agreement between dates and data from other analytical methods, such as sedimentol, palynol, and Dinoflagellae studies, even for samples with possible detritic carbonates. Only Ly-2491 seems too recent for unknown reasons. Results from Cores MKS1 and BRK were not able to be interpreted.

TABLE 21

NW Mediterranean Sea

\begin{tabular}{cccccc}
\hline $\begin{array}{c}\text { Sample } \\
\text { no. }\end{array}$ & Core & Geog coordinates & $\begin{array}{c}\text { Depth } \\
(\mathrm{cm})\end{array}$ & DR & $\begin{array}{c}\text { Age } \\
(\mathrm{BP})\end{array}$ \\
\hline Ly-2493 & MKS 1 & $\left(42^{\circ} 59^{\prime} \mathrm{N}, 5^{\circ} 12^{\prime} \mathrm{E}\right)$ & $44-54$ & 0.5 & $13,450 \pm 290$ \\
-2547 & MKS 7 & $\left(42^{\circ} 51^{\prime} \mathrm{N}, 5^{\circ} 04^{\prime} \mathrm{E}\right)$ & $10-20$ & 0.7 & $14,260 \pm 260$ \\
-2548 & MKS 7 & $\left(42^{\circ} 51^{\prime} \mathrm{N}, 5^{\circ} 04^{\prime} \mathrm{E}\right)$ & $105-110$ & 1 & $26,420 \pm 550$ \\
-2549 & MKS 7 & $\left(42^{\circ} 51^{\prime} \mathrm{N}, 5^{\circ} 04^{\prime} \mathrm{E}\right)$ & $195-205$ & 1 & $29,730 \pm 750$ \\
-2545 & KSPF 80-04 & $\left(42^{\circ} 21^{\prime} \mathrm{N}, 4^{\circ} 55^{\prime} \mathrm{E}\right)$ & $90-97$ & 0.7 & $11,080 \pm 220$ \\
-2546 & KSPF 80-04 & $\left(42^{\circ} 21^{\prime} \mathrm{N}, 4^{\circ} 55^{\prime} \mathrm{E}\right)$ & $130-142$ & 0.7 & $21,230 \pm 430$ \\
-2543 & KSPF 79-02 & $\left(42^{\circ} 01^{\prime} \mathrm{N}, 5^{\circ} 13^{\prime} \mathrm{E}\right)$ & $30-40$ & 0.6 & $10,760 \pm 240$ \\
-2544 & KSPF 79-02 & $\left(42^{\circ} 01^{\prime} \mathrm{N}, 5^{\circ} 13^{\prime} \mathrm{E}\right)$ & $65-75$ & 0.8 & $23,180 \pm 470$ \\
-2489 & K 30-30 & $\left(41^{\circ} 49^{\prime} \mathrm{N}, 8^{\circ} 24^{\prime} \mathrm{E}\right)$ & $10-20$ & 1 & $15,720 \pm 300$ \\
-2490 & $\mathrm{~K} \mathrm{30-30}$ & $\left(41^{\circ} 49^{\prime} \mathrm{N}, 8^{\circ} 24^{\prime} \mathrm{E}\right)$ & $29-39$ & 0.8 & $31,300 \pm 1200$ \\
-2557 & $\mathrm{~K} \mathrm{30-30}$ & $\left(41^{\circ} 49^{\prime} \mathrm{N}, 8^{\circ} 24^{\prime} \mathrm{E}\right)$ & $50-60$ & 0.3 & $\geq 34,600$ \\
-2491 & $\mathrm{~K} \mathrm{30-30}$ & $\left(41^{\circ} 49^{\prime} \mathrm{N}, 8^{\circ} 24^{\prime} \mathrm{E}\right)$ & $60-70$ & 1 & $31,700 \pm 1000$ \\
-2492 & $\mathrm{~K} \mathrm{30-30}$ & $\left(41^{\circ} 49^{\prime} \mathrm{N}, 8^{\circ} 24^{\prime} \mathrm{E}\right)$ & $130-140$ & 1 & $\geq 37,000$ \\
-2556 & $\mathrm{BRK}$ & Undetermined & & 0.5 & $16,560 \pm 330$ \\
\hline
\end{tabular}

Ly-2951. Le Marin, Martinique

$29,000 \pm 720$

Coral outcrops at several $\mathrm{m}$ above $\mathrm{msl}$ in Le Marin Bay $\left(14^{\circ} 28^{\prime} \mathrm{N}\right.$, $60^{\circ} 53^{\prime} \mathrm{W}$ ). Coll 1982 by A Klingebiel and R Assor and subm 1983 by C 
Carruesco. Comment (CC): date is older than expected, and indicates local neotectonic influence.

Ly-2898. Sidi El Fallagui, Bizerte Dept, N Tunisia

Marine or lagoonal shells from sandy marl and clayey horizon, outcropping at alt ca $60 \mathrm{~m}, 3 \mathrm{~km}$ from shore $\left(41^{\circ} 45^{\prime} \mathrm{N}, 9^{\circ} 40^{\prime} \mathrm{E}\right)$. Coll and subm 1982 by A Miossec, Lab Geog, Univ Nantes $(0.3 \mathrm{dr})$. Comment (AM): date indicates sediments date from at least Tyrrenian transgression; alts show that tectonic deformation has been marked since this period (Miossec, 1977).

\section{ARCHAEOLOGIC SAMPLES}

Historic Period

France

\section{$\delta^{14} \mathrm{C}=-8 \pm 12 \%$}

Ribs of large horse found in pit in Gallic sanctuary $\left(49^{\circ} 29^{\prime} \mathrm{N}, 2^{\prime} 40^{\circ} \mathrm{E}\right)$. Coll 1980 by JL Brunaux and P Méniel; subm 1983 by P Méniel and F Poplin, Mus Hist Nat Paris. Sample subm to determine horse's origin. Archaeol data suggest horse belonged to Gallic level (Brunaux, Méniel, \& Rapin, 1980); osteol data (mainly large size) favors 19th century. Comment (FP): date confirms later hypothesis, $i e$, bone is probably from horse of one of Franco-German wars.

\section{Bayeux tapestry series, Bayeux, Calvados}

Linen thread from back of Bayeux tapestry from Bayeux Mus $\left(49^{\circ} 17^{\prime} \mathrm{N}, 0^{\circ} 42^{\circ} \mathrm{W}\right)$. Coll 1982 and subm 1983 by $\mathrm{F}$ Macé de Lépinay, Hist Monuments Dept, Paris, as part of study of known Medieval "Queen Matilda tapestry" which is actually wool embroidered on linen.

\section{Ly-3047. Fragment de doublure}

$390 \pm 120$

$*_{A D 1385-1635}$

Threads from piece of lining added during repair $(0.7 \mathrm{dr})$. Corrected date interval from Stuiver (1982): *AD1300 to 1660 .

\section{Ly-3048. Fil de bagage}

$260 \pm 90$

*AD1425-1950

Threads from attached lining of tapestry. Stuiver corrected date interval: *AD1420 to 1950 .

General Comment (FM de L): as expected, study of threads and linen both confirm that repairs occurred long after embroidery (very well-dated to end of 11 th century). Large date range obtained after applying both types of correction intervals does not exclude extreme end of Middle Ages as date of weaving of lining, nor 19th century for its final repair. 


\section{Dognon series, Le Châtenet en Dognon, Haute-Vienne}

Charcoal from two levels in former bldg near Pont du Dognon $\left(45^{\circ} 56^{\prime} \mathrm{N}, 1^{\circ} 30^{\prime} \mathrm{E}\right)$. Coll 1982 by G Cantié and subm by JM Desbordes, Dir Antiquités prehist, Limoges.

Ly-3145. Le Dognon, JI-1/2

Sample from pit, subm 1984.

\section{Ly-3004. Le Dognon, KI-1}

Sample from hearth, subm 1983.

\section{Ly-3005. Le Dognon, SI-1}

Sample from silo, subm 1983.
$770 \pm 100$

$*_{A D 1050-1345}$

$790 \pm 100$

$*_{A D 1040-1335}$

$960 \pm 120$

$*_{A D 885-1245}$

General Comment (JMD): dates are within same statistical margins; indicating 11 th century, $i e$, beginning of date range (11th-15th centuries) expected according to typology of assoc ceramics (Cantié, in press).

\section{Ly-2438. Le Moléron-Sablons, Décines, Rhône}

$680 \pm 130$

*AD 1 140-1420

Charcoal from habitation level at base of feudal mound $\left(45^{\circ} 47^{\prime} \mathrm{N}\right.$, $4^{\circ} 59^{\prime} \mathrm{E}$ ). Coll and subm 1981 by G Marien and JM Monnier, Meyzieu. Comment $(\mathrm{JM})$ : date is a little younger than that suggested by 10 th century coins in same level.

\section{Coyroux series, Aubazine, Corrèze}

Charcoal from two superimposed levels in terrace built as basement of Cistercian abbey (Barrière, 1983) $\left(45^{\circ} 10^{\prime} \mathrm{N}, 1^{\circ} 40^{\prime} \mathrm{E}\right)$. Coll 1981 and subm 1983 by JM Desbordes.

Ly-3006. Coyroux-Aubazine, M-18

$780 \pm 170$

From upper level $(0.3 \mathrm{dr})$.

$*_{A D} 935-1405$

\section{Ly-3007. Coyroux-Aubazine, H-17}

$1100 \pm 100$

From lower level.

General Comment (JMC): Ly-3007 agrees with period of abbey's expansion (12th century). Ly-3006 shows relatively old date for beginning of terrace construction.

\section{Ly-2554. Carrière de Beaulieu, Bardouville, Seine Maritime}

$820 \pm 120$

*AD 1030-1325

Human bones from sand quarry near Late Neolithic collective sepulture (Graindor, 1966) $\left(49^{\circ} 26^{\circ} \mathrm{N}, 0^{\circ} 51^{\prime} \mathrm{E}\right)$. Coll and subm 1981 by G Ver- 
ron, Dir Antiquités Prehist, Caen. Comment (GV): date shows inhumation occurred long after sand was deposited and neighboring Neolithic burials took place; latter were previously dated, Ly-2348: $4550 \pm 130(\mathrm{R}, 1983$, $\mathrm{v} 25, \mathrm{p} 101)$.

\section{Ly-2665. Le-Haut-du-Château, Aingeray,} Meurthe et Moselle

Charcoal from Layer C, lowest level of test excavation in fill of barredspur $\left(48^{\circ} 45^{\prime} \mathrm{N}, 6^{\circ} 0^{\prime} \mathrm{E}\right)$. Coll 1981 and subm 1982 by A Lieger, Toul. Comment (AL): two dates were expected, according to ancient texts and structure of fortification-either Late Bronze age or end of Gallo-Roman period. Date represents much later occupation.

\section{La Folie series, Meunes, Loir et Cher}

Charcoal from lime plaster kiln of primitive type found in sandy alluvia of Le Cher R ( $\left.47^{\circ} 15^{\prime} \mathrm{N}, 1^{\circ} 28^{\prime} \mathrm{E}\right)$. Coll 1981 and subm 1982 by J Despriée, Dir Antiquités Prehist, Orléans. No assoc industry.

\section{Ly-2702. La Folie P4}

$1030 \pm 150$

$*_{A D 655-1250}$

\section{Ly-2703. La Folie P5}

$820 \pm 150$

$*_{A D} 910-1385$

General Comment (JD): primitive nature of kiln suggested relatively old age, eg, contemporaneous with neighboring sites of "Les Champs d'Urnes" period (Late Bronze age). Date shows kiln is Medieval.

\section{Ly-3008. Champmain, Saint-Léonard de Noblat Haute-Vienne}

$1190 \pm 150$

*AD 595-1055

Charcoal from tomb $\left(45^{\circ} 50^{\prime} \mathrm{N}, 1^{\circ} 29^{\prime} \mathrm{E}\right)$. Coll 1981 by M Tandeau de Marsac and subm 1982 by JM Desbordes; assoc with less characteristic Medieval ceramics (Tandeau de Marsac, 1982). Comment (JMD): date shows assoc potsherds are Carolingian in age.

\section{Colletière series, Charavines, Isère}

Samples from Layer II of Paladru Lake sediments, in area of submerged village site $\left(45^{\circ} 25^{\prime} \mathrm{N}, 5^{\circ} 30^{\prime} \mathrm{E}\right)$. Coll 1977 and subm 1979 by $\mathrm{M}$ Collardelle, Archaeol Hist Mus, Grenoble. Coins found on site as well as typol of assoc material and hist evidence indicate settlement of village occurred during 11 th century (Collardelle, 1980).

\section{Ly-1869. Colletière, charcoal}

Ly-1870. Colletière, bark
$1240 \pm 140$

*AD $570-1030$

$1200 \pm 150$

$*_{A D} 590-1050$ 


\section{Ly-1959. Colletière, seeds}

$$
900 \pm 110
$$

$*_{A D 910-1270}$

General Comment (MC): only Ly-1959 agrees exactly with hist age; Ly-1870 and -1869 remain unexplained, as they do not fit dendrochronol data indicating more recent date for wood at site (ca 9th or 11 th century). However, this deviation is reduced with corrected dates (Evin \& Olive, in press).

\section{La Dent series, Meyzieu, Rhône}

Human bones from several graves in cemetery $\left(45^{\circ} 47^{\prime} \mathrm{N}, 5^{\circ} 0^{\prime} \mathrm{E}\right)$. Coll 1979 and subm 1980 and 1981 by J Monnier.

General Comment (JM): although 1 st 3 graves were superimposed, all dates except Ly-2330 are very close, suggesting relatively short period for all burials in necropolis, corresponding to "Burgonde" period, or beginning of High Middle Ages, as had been expected from osteol of skeletons.

TABLE 22

La Dent

\begin{tabular}{|c|c|c|c|c|}
\hline $\begin{array}{c}\text { Sample } \\
\text { no. }\end{array}$ & $\begin{array}{l}\text { Ref } \\
\text { no. }\end{array}$ & DR & $\begin{array}{c}\text { Age } \\
\text { (BP) }\end{array}$ & $\begin{array}{l}\text { Corrected } \\
\text { date } \\
\text { interval } \\
\text { (AD) }\end{array}$ \\
\hline Ly-2229 & T 37A & 1 & $1480 \pm 90$ & $350-650$ \\
\hline $\begin{array}{l}-2230 \\
\end{array}$ & T 37B & 1 & $1480 \pm 90$ & $350-650$ \\
\hline-2231 & $\mathrm{~T} 37 \mathrm{C}$ & 1 & $1330 \pm 110$ & $570-885$ \\
\hline-2669 & Т 43 & 0.7 & $1370 \pm 150$ & $415-895$ \\
\hline-2670 & $\mathrm{~T} 7$ & 1 & $1290 \pm 140$ & $465-1015$ \\
\hline
\end{tabular}

\section{Cemeteries series, Tours, Indre et Loire}

Human bones from several graves in two cemeteries $\left(47^{\circ} 22^{\prime} \mathrm{N}\right.$, $0^{\circ} 40^{\prime} \mathrm{E}$ ) coll 1981 by Lab Archéol Urbaine and subm 1981 by H Galinié, Tours.

General Comment (HG): dates are within same statistical margin and differences in dr of all samples reflect variation in preservation of organic matter in open-air sites. As expected (Galinié \& Thureau, 1976), both graves from Saint-Pierre le Puellier cemetery are dated to end of Middle Ages. However, according to archaeol data, which attributes them to 11 th or 12 th centuries, Ly-2659 is too young by ca $200 \mathrm{yr}$, and Ly-2660 fits only if its large

TABLE 23

Tours cemeteries

\begin{tabular}{clcccc}
\hline $\begin{array}{c}\text { Sample } \\
\text { no. }\end{array}$ & \multicolumn{1}{c}{ Site } & Ref no. & DR & Age (BP) & $\begin{array}{c}\text { Corrected } \\
\text { interval } \\
\text { date } \\
\text { (AD) }\end{array}$ \\
\hline $\begin{array}{c}\text { Ly-2659 } \\
-2660\end{array}$ & St Pierre le Puellier & $1-S-269$ & 1 & $640 \pm 100$ & $1235-1415$ \\
-2661 & St Pierre le Puellier & $1-S-372$ & 0.1 & $720 \pm 220$ & $920-1480$ \\
-2662 & Rue des Ursulines & $6-S-2$ & 1 & $1570 \pm 130$ & $160-630$ \\
\hline
\end{tabular}


statistical margin is considered. Both dates from La Rue des Ursulines cemetery are within expected range (4th to 8th centuries). Archaeol evidence attributes Ly-2661 to 4th or 5th century and Ly-2662 was assumed to be from 6 th to 8 th centuries.

\section{Ly-3039. Maison-Rouge, Marvejols, Lozère}

$1460 \pm 100$

$*_{A D} 400-755$

Human bones from Grave 15 of cemetery $\left(44^{\circ} 33^{\prime} \mathrm{N}, 3^{\circ} 19^{\prime} \mathrm{E}\right)$. Coll 1982 and subm 1983 by J Thomas-Beeching, Marvejols. Comment (JTB): date agrees with expected age based on Merovingian style (beginning of Middle Ages) of tombs in region.

\section{Ly-2855. Village d'Agos, Agos-et-Vildalos, $\quad 1450 \pm 160$ Hautes-Pyrénées \\ *AD250-865}

Bones of domestic animals from hearth revealed by excavations near cemetery from High Middle Ages $\left(43^{\circ} 2^{\prime} \mathrm{N}, 0^{\circ} 4^{\prime} \mathrm{W}\right)$. Coll 1981 by R Vié and subm 1982 by A Clot, Bordères/Echez $(0.7 \mathrm{dr})$. Comment (AC): date shows that hearth could not have been part of cemetery (Coquerel \& Pousthamis, 1977) although it is later than expected from assoc rare Roman tiles (Tegulae) (Vié, Koutnetzoff, \& Clott, 1983).

\section{Albigny-Condion series, Seyssel, Haute-Savoie}

Human bones from several graves in necropolis surrounding funerary basilica $\left(47^{\circ} 57^{\prime} \mathrm{N}, 5^{\circ} 50^{\prime} \mathrm{E}\right)$. Coll 1980 and subm 1982 by B Bizot, Dir Antiquités Hist, Lyon, and J Serralongue, Centre Archéol Annecy.

\section{Ly-2866. Sepulture S-50}

$1080 \pm 130$

From SE part of necropolis ( $0.8 \mathrm{dr})$. Expected age: 7 th century.

\section{Ly-2865. Sepulture S-89}

$1390 \pm 90$

From outside and near $\mathrm{W}$ wall of basilica. Expected age: 6 th to 9 th centuries.

\section{Ly-2864. Sepulture S-90}

$1420 \pm 120$

From inside and near $\mathrm{N}$ gate of basilica. Expected age: 5 th to 7 th centuries.

\section{Ly-2863. Sepulture S-48}

$1720 \pm 130$

From small necropolis ca $50 \mathrm{~m}$ from basilica $(0.7 \mathrm{dr})$. Expected age: $3 \mathrm{rd}$ to 5 th centuries.

General Comment (BB \& JS): all dates are in expected range from assoc archaeol material (Bizot \& Serralongue, in press). They confirm long use of site and much older age of small adjacent necropolis (Ly-2863). 


\section{Ly-2955. Sainte-Colombe le Vieux, Sainte-Colombe, $\quad 1730 \pm 150$ Rhône \\ *1BC-AD584}

Human bones from Grave no. 9 of cemetery $\left(45^{\circ} 31^{\prime} \mathrm{N}, 4^{\circ} 50^{\prime} \mathrm{E}\right)$. Coll 1981 by B Hély and subm 1983 by A Cogoluènhes, Lab Geol, Univ Lyon I $(0.7 \mathrm{dr})$. Necropolis is near Gallo-Roman city, Saint-Romain-en-Gal, former quarter of Vienne. Comment (AC): date confirms that necropolis belonged to Gallo-Roman site, despite lack of assoc archaeol material.

\section{La Place series, Villiers-le-Sec, Val d'Oise}

Bones from animal sp from refuse pit of house $\left(49^{\circ} 5^{\prime} \mathrm{N}, 2^{\circ} 23^{\prime} \mathrm{E}\right)$. Coll 1981-82 and subm 1982 by R Guadagnin, Arts et Traditions Populaires Mus, Paris.

Ly-2599. Villiers le Sec, No. 2

$1010 \pm 160$

From Pit $2(0.5 \mathrm{dr})$.

*AD665-1235

Ly-2598. Villiers le Sec, No. 1

$1930 \pm 150$

From Pit 1 (0.5 dr).

*370BC-AD360

\section{Ly-2728. Villiers le Sec, No. 1b}

$1810 \pm 150$

From Pit 1 ( $0.5 \mathrm{dr})$.

*150BC-AD550

General Comment (RG): Ly-2599 agrees perfectly with assumed age of house (7th century). Ly-2728 from 1982 excavation was to check Ly-2598 which appeared aberrant. Both dates were later confirmed by data from 1983 excavation, which revealed refuse pit from High Roman Empire (1 st or 2nd century AD) with Sigillate ceramics and fibula (Guadagnin, in press).

\section{Saône Boats series, Châlon sur Saône, Saône et Loire}

Wood from remains of boats submerged in Saône $\mathrm{R}$, coll by river dredging, Châlon-sur-Saône from 1977 to 1981 and subm 1982 by L Bonnamour, Denon Mus, Châlon-sur-Saône.

General Comment (LB): dates show evolution in boat form, which for centuries changed little (Bonnamour, 1978-79, 1980, 1981).

TABLE 24

Saône boats

\begin{tabular}{ccclccc}
\hline $\begin{array}{c}\text { Sample } \\
\text { no. }\end{array}$ & Site & Geog coordinates & Origin of wood & DR & $\begin{array}{c}\text { Age } \\
(\mathrm{BP})\end{array}$ & $\begin{array}{c}\text { Corrected } \\
\text { date } \\
\text { interval }\end{array}$ \\
\hline Ly-2743 & Ormes & $\left(46^{\circ} 38^{\prime} \mathrm{N}, 4^{\circ} 56^{\prime} \mathrm{E}\right)$ & Monoxylic barge & 1 & $720 \pm 120$ & AD1180-1400 \\
-2744 & Ormes & $\left(46^{\circ} 38^{\prime} \mathrm{N}, 4^{\circ} 56^{\prime} \mathrm{E}\right)$ & Boat timber & 0.6 & $990 \pm 170$ & AD775-1260 \\
-2742 & Lux & $\left(46^{\circ} 45^{\prime} \mathrm{N}, 4^{\circ} 52^{\prime} \mathrm{E}\right)$ & Helm oar & 1 & $1910 \pm 120$ & $160 \mathrm{BC}-\mathrm{AD} 245$ \\
-2741 & Bragny & $\left(46^{\circ} 54^{\prime} \mathrm{N}, 5^{\circ} 03^{\prime} \mathrm{E}\right)$ & Boat timber & 1 & $1920 \pm 130$ & $365 \mathrm{BC}-\mathrm{AD} 380$ \\
\hline
\end{tabular}




\section{Ateliers Municipaux series, Saintes, Charente Maritime}

Human bones from two levels in funeral pit from Gallo-Roman villa $\left(45^{\circ} 45^{\circ} \mathrm{N}, 0^{\circ} 37^{\prime} \mathrm{W}\right)$. Coll $1970-72$ by $\mathrm{M}$ Rouvreau and subm 1983 by $\mathrm{M}$ Collillieux, Lab Anthropol, Univ Caen.

Ly-3024. Saintes No. 7

$1990 \pm 110$

From 6 to $8 \mathrm{~m}$ depth in upper level of bone fill of pit $(0.7 \mathrm{dr})$.

Ly-3025. Saintes No. 8

$2030 \pm 70$

From 8 to $20 \mathrm{~m}$ depth in lower level of bone fill of pit.

General Comment (MC): both dates are within same statistical margins, indicating rapid filling rate for all $12 \mathrm{~m}$ of Roman sediments in pit. They agree well with expected age (Maurin, 1978), 1st or 2nd century AD.

\section{Protohistoric Period}

\section{Africa}

\section{Ly-3028. Bekrat, El Goléa, Laghouat Willava,} Algeria

$$
\delta^{14} \mathrm{C}=26.5 \pm 15.7 \%
$$

Charcoal from ca $15 \mathrm{~m}$ depth, under sandy level containing ostrich egg fragments $\left(30^{\circ} 50^{\prime} \mathrm{N}, 2^{\circ} 45^{\prime} \mathrm{E}\right)$. Coll 1982 by M Montanari and subm 1983 by R Leclerc, El Goléa. Comment (RL): age corresponding to presence of ostrich in area was expected, but date shows site was disturbed.
Ly-2528. Babanki-Tungo, Le Mézam Dept, Cameroun
Modern
$\delta^{14} \mathrm{C}=0.0 \pm 16.0 \%$ o

Charcoal from base of $4 \mathrm{~m}$ deposit of scoria near smelting furnace at Agric Sta $\left(5^{\circ} 58^{\prime} \mathrm{N}, 10^{\circ} 20^{\prime} \mathrm{E}\right)$. Coll 1980 and subm 1981 by JP Warnier, Univ Yaoundé. Comment (JPW): date and strat position of sample shows $4 \mathrm{~m}$ accumulation of scoria occurred quite rapidly.

\section{Ly-2817. Grand Jacques, Ivory Coast}

$750 \pm 160$

$*_{A D 1030-1410}$

Charcoal from $2.1 \mathrm{~m}$ in depth of boring in shelly kitchen midden on beach strand at ca $50 \mathrm{~m}$ from coast $\left(5^{\circ} 10^{\prime} \mathrm{N}, 4^{\circ} 31^{\prime} \mathrm{E}\right)$. Coll and subm 1982 by J Rivallain, Univ Abidjan. Change in style of assoc ceramics in level suggests change in human population. Comment (JR): date is older than assumed from oral histories (Rivallain, 1983).

$820 \pm 110$

Ly-2687. Bagamoyo, Labattoir, Mayotte Comores I. $\quad *_{A D} 1035-1325$

Human bones from Grave 9 of large necropolis, partly submerged on La Petite Terre I. coast $\left(12^{\circ} 47^{\prime} \mathrm{S}, 45^{\circ} 15^{\prime} \mathrm{E}\right)$. Coll 1981 and subm 1982 by $\mathrm{C}$ Allibert, Lyon, A Argant, and J Argant, Bron. Assoc with few scattered pot- 
sherds. Comment (AA): date precisely confirms age expected from ceramics, 11 th or 13th centuries, Hagnoundou period (Allibert, Argant, \& Argant, 1983).

\section{House of Koumbi Saleh city, Timbedra, Mauritania}

Samples from several archaeol levels of house in S Central area of main tell of Koumbi Saleh $\left(15^{\circ} 46^{\circ} \mathrm{N}, 7^{\circ} 59^{\prime} \mathrm{W}\right)$. Site was supposedly capital of kingdom of Ghana. Excavations on site were directed by S Robert, Mauritanian Inst Sci Research; house was excavated 1975, 1976 by S Berthier, Univ Lyon II, who coll samples previously pub (R, 1979, v 21, p 430), and coll and subm 1980 following samples (tables 25a, b). House was probably occupied continuously for six centuries according to following strat:

TABLE 25a

Stratigraphy of House of Koumbi Saleh

\begin{tabular}{|c|c|c|c|}
\hline Level & Archaeol event & $\begin{array}{l}\text { Expected date } \\
\text { (century) }\end{array}$ & Previous dates \\
\hline $\mathrm{V}$ & $\begin{array}{l}\text { Abandonment and } \\
\text { destruction }\end{array}$ & $\begin{array}{l}\text { End of } 14 \text { th } / \text { or } \\
\text { beginning of } \\
15 \text { th }\end{array}$ & \\
\hline IV & & & $\begin{array}{r}\text { Ly-1521:230 } \pm 120 \\
-1525: 440 \pm 180\end{array}$ \\
\hline IVa, b & End of max occupation & 14 th & $-1524: 550 \pm 230$ \\
\hline III & Max prosperity of city & 13th & $-1526: 860 \pm 210$ \\
\hline II & Max city development & $\begin{array}{l}\text { End of } 11 \text { th } \\
\text { beginning of } \\
\text { 12th }\end{array}$ & $\begin{array}{l}-1341: 1000 \pm 150 \\
-1520: 590 \pm 120\end{array}$ \\
\hline I & Short period of 1 st bldg & Middle of 11 th & \\
\hline $\mathrm{O}$ & Refuse pit in basal soil & $\begin{array}{l}\text { End of } 9 \text { th or } \\
10 \text { th }\end{array}$ & \\
\hline
\end{tabular}

TABLE 25b

House of Koumbi Saleh

\begin{tabular}{|c|c|c|c|c|c|c|c|c|}
\hline $\begin{array}{c}\text { Sample } \\
\text { no. }\end{array}$ & $\begin{array}{l}\text { Ref } \\
\text { no. }\end{array}$ & Level & $\begin{array}{l}\text { Depth } \\
(\mathrm{cm})\end{array}$ & Sample & DR & $\begin{array}{l}\text { Age } \\
\text { (BP) }\end{array}$ & $\begin{array}{l}\text { Age } \\
\text { (AD) }\end{array}$ & $\begin{array}{l}\text { Corrected } \\
\text { date } \\
\text { interval } \\
\text { (AD) }\end{array}$ \\
\hline Ly-2509 & SB III $41 / 48$ & V & $190-215$ & Potsherd & 0.5 & $720 \pm 140$ & 1230 & $1040-1415$ \\
\hline-2510 & SB III $67^{\prime}$ & V & $190-215$ & Charcoal & 1 & $530 \pm 140$ & 1420 & $1245-1635$ \\
\hline-2540 & SB III 80 & IVb & $140-250$ & Charcoal & 1 & $1020 \pm 150$ & 930 & $660-1250$ \\
\hline-2539 & SB III 101 & IVa & $295-310$ & Charcoal & 1 & $530 \pm 100$ & 1420 & $1275-1500$ \\
\hline-2507 & SB III 131 & III & $315-320$ & Potsherd & 1 & $750 \pm 130$ & 1500 & $1030-1410$ \\
\hline-2508 & SB III 130 & III & $315-320$ & Charcoal & 0.6 & $780 \pm 100$ & 1170 & $1045-1315$ \\
\hline-2506 & SB III 247 & IIb & $430-435$ & Charcoal & 1 & $710 \pm 100$ & 1240 & $1195-1400$ \\
\hline-2505 & SB III 249 & IIb & $445-450$ & Potsherd & 0.2 & $810 \pm 170$ & 1140 & $915-1395$ \\
\hline-2538 & SB III 195 & IIa & $460-470$ & Charcoal & 1 & $790 \pm 120$ & 1160 & $1040-1335$ \\
\hline-2537 & SB III 265 & IIa & $490-510$ & Charcoal & 0.5 & $880 \pm 170$ & 1070 & $890-1330$ \\
\hline-2536 & SB III 210 & I & $520-530$ & Charcoal & 1 & $850 \pm 100$ & 1100 & $935-1315$ \\
\hline-2535 & SB III 271 & I & $530-565$ & Charcoal & 1 & $800 \pm 100$ & 1150 & $1040-1335$ \\
\hline-2534 & SB III 274 & I & $560-570$ & Cha & 1 & $730 \pm 140$ & 1220 & $1035-1415$ \\
\hline-3147 & SB IV 30 & $\mathrm{O}$ & & Charcoal & 1 & $1620 \pm 150$ & 330 & $60-620$ \\
\hline-2533 & SB III 289 & $\mathrm{O}$ & $600-610$ & Charcoal & 1 & $770 \pm 150$ & 1180 & $1020-1405$ \\
\hline-2532 & SB III 287 & $\mathrm{O}$ & $600-625$ & Charcoal & 1 & $870 \pm 140$ & 1080 & $920-1290$ \\
\hline-2504 & SB III 295 & $\mathrm{O}$ & $620-650$ & Charcoal & 0.7 & $950 \pm 130$ & 1000 & $855-1285$ \\
\hline-2503 & SB III 305/306 & $\mathrm{O}$ & $620-670$ & Potsherd & 0.2 & Modern: & ${ }^{14} \mathrm{C}=$ & $\pm 18 \%$ \\
\hline-3146 & SB IV 35 & $\mathrm{O}$ & & Charcoal & 1 & $1270 \pm 90$ & 680 & $590-905$ \\
\hline
\end{tabular}


General Comment (SB): results agree better with strat and expected ages than previous series, samples of which were probably too small. Ly-3147, -2540 , and -3146 are outside general trend by ca $400 \mathrm{yr}$, or more (Ly-3147) and remain so even after correction. This may indicate use of ancient wood as fuel at time of destruction of city as was frequent, especially in arid regions (see, eg, Les Kellia Qoucour Isa; Ly-267: $1645 \pm 80$ and B-988: $1530 \pm 100$ ( R, 1971, v 13, p 55). Dates obtained from potsherds either agree exactly with those from charcoal (Ly-2505, -2538), are somewhat different, but within statistical margin (Ly-2509,-2507), or are completely erratic for unknown reasons (Ly-2503). All dates from Level III, IV, and V are comparable to those from equivalent archaeol levels in neighboring sites excavated by S Robert (R, 1979, v 21, p 430-431), or excavated by A Cros and later pub $(\mathrm{R}, 1977, \mathrm{v} 19, \mathrm{p} 162)$. They confirm that at least area of excavated house was not occupied before end of 10 th century, $i e$, Mauny's (1951) Last Pre-Islamic period (Berthier, 1983).

\section{Mutwarubona series, Ndora, Rwanda}

Charcoal from $50 \mathrm{~cm}$ depth in remains of two smelting furnaces $\left(2^{\circ} 36^{\prime} \mathrm{S}, 29^{\circ} 48^{\prime} \mathrm{E}\right)$. Coll and subm 1982 by F Van Noten, Mus Royal Afrique Centrale, Tervuren, Belgium.

\section{Ly-2668. Haut-Fourneaux 2}

$1380 \pm 170$

From smelting furnace No. 2 (0.6 dr).

$*_{A D 245-1005}$

\section{Ly-2667. Haut-Fourneaux 1}

$2020 \pm 330$

From smelting furnace No. 1 ( $0.65 \mathrm{dr})$.

$* 760 B C-A D 565$

General Comment (FVN): despite wide statistical margin, both dates agree with expected date range (1BC to $\mathrm{AD} 700$ ), as smelting furnaces were in use since Early Iron age (Van Noten, 1983).

\section{Akagéra National Park, Kinbungo, Mutata Prov, Rwanda}

Charcoal from several sites in park: N'Dama Cave $\left(2^{\circ} 23^{\prime} \mathrm{S}, 30^{\circ} 26^{\prime} \mathrm{E}\right)$, Muhororo rock shelter $\left(2^{\circ} 54^{\prime} \mathrm{S}, 30^{\circ} 31^{\prime} \mathrm{E}\right)$ and Mucucu II rock shelter $\left(2^{\circ} 35^{\prime} \mathrm{N}, 30^{\circ} 30^{\prime} \mathrm{E}\right)$. Coll 1978 (Ly-2798) and 1980 and subm 1980 by B Lugan, Hist Dept, Univ Lyon III. Assoc industries were ceramics of recent rolled type, eg, at N'Dama (Lugan, Sirven, \& Vérin, 1979), and flint microliths of Wiltonian type, eg, at Muhuroro (Lugan, 1983). Samples indicate occupation from Late Stone age (LSA) with ceramics from end of Early Iron age (EIA) of Uréwé type, ending with Late Iron age (LIA) with rolled ceramics and microliths.

General Comment (BL): Ly-2798 shows that site occupation is only recent. Ly-2382 suggests that majority of microlith industries date from ca $2000 \mathrm{BP}$ or just before EIA. All dates for Mucucu II site agree with age expected from assoc industries, archaeol attribution, and strat despite limited 
TABLE 26

Akagéra National Park

\begin{tabular}{|c|c|c|c|c|c|c|c|}
\hline $\begin{array}{c}\text { Sample } \\
\text { no. }\end{array}$ & Site & $\begin{array}{l}\text { Sq or } \\
\text { level }\end{array}$ & $\begin{array}{c}\text { Depth } \\
\text { (cm) }\end{array}$ & $\begin{array}{c}\text { Assoc } \\
\text { industry }\end{array}$ & DR & $\begin{array}{l}\text { Age } \\
\text { (BP) }\end{array}$ & $\begin{array}{l}\text { Corrected } \\
\text { date } \\
\text { interval or } \\
\text { activity }\end{array}$ \\
\hline Ly-2798 & N'Dama & $\begin{array}{l}\text { Base } \\
\text { level }\end{array}$ & 110 & Potsherd & 0.7 & Modern & $\delta^{14} \mathrm{C}=+20 \pm 16 \%$ \\
\hline-2450 & Muhororo & Sq 1 & $20-25$ & $\begin{array}{l}\text { Potsherd and } \\
\text { microliths }\end{array}$ & 0.10 & $350 \pm 200$ & AD1305-1950 \\
\hline-2382 & Muhororo & Sq 1 & $55-65$ & $\begin{array}{l}\text { Potsherd and } \\
\text { microliths }\end{array}$ & 0.3 & $1970 \pm 190$ & $400 \mathrm{BC}-\mathrm{AD} 410$ \\
\hline-2449 & Mucucu II & $\mathrm{Sq} 1$ & $10-20$ & L I A & 0.10 & $1220 \pm 220$ & AD460-1195 \\
\hline-2235 & Mucucu II & Sq 1 & $20-30$ & E I A & 0.15 & $2380 \pm 270$ & $1095 \mathrm{BC}-\mathrm{AD} 200$ \\
\hline-2383 & Mucucu II & $\mathrm{Sq} 2 \mathrm{a}$ & $30-35$ & LS A & 0.6 & $2040 \pm 180$ & $415 \mathrm{BC}-\mathrm{AD} 250$ \\
\hline-2385 & Mucucu II & $\mathrm{Sq} 2,3$ & $30-35$ & $\mathrm{LSA}$ & 0.2 & $1210 \pm 160$ & AD590-1050 \\
\hline-2384 & Mucucu II & $\mathrm{Sq} 2,3 \mathrm{a}$ & $40-45$ & L S A & 0.25 & $2020 \pm 200$ & $410 \mathrm{BC}-\mathrm{AD} 335$ \\
\hline-2236 & Mucucu II & $\mathrm{Sq} 1$ & ca 45 & LS A & 0.5 & $1880 \pm 170$ & $375 \mathrm{BC}-\mathrm{AD} 555$ \\
\hline-2237 & Mucucu II & Sq 1 & $45-55$ & L S A & 0.10 & $3940 \pm 320$ & $3155-1765 \mathrm{BC}$ \\
\hline-2386 & Mucucu II & Sq 3 & $50-55$ & L S A & 0.6 & Modern & $\delta^{14} \mathrm{C}=+3 \pm 22 \%$ \\
\hline-2387 & Mucucu II & $\mathrm{Sq} 2 \mathrm{a}$ & $50-60$ & $\mathrm{LS} \mathrm{A}$ & 0.07 & $2620 \pm 320$ & $1395 \mathrm{BC}-\mathrm{AD} 170$ \\
\hline
\end{tabular}

amount of dating material. Ly-2449 shows relatively early age for LIA whereas 1st appearance of ceramics occurs at ca $2000 \mathrm{BP}$, as suggested by Ly-2235 and -2383 .

\section{Oceania}

\section{Ly-2310. La Roche, Maré I., New Caledonia}

$1040 \pm 110$

$*_{A D} 790-1215$

Human bones from collective grave in open-air site near airport $\left(21^{\circ} 28^{\prime} \mathrm{S}, 168^{\circ} 2^{\prime} \mathrm{E}\right)$. Coll 1977 by J P Maitre and subm 1980 by D Frimigacci, Office Recherche Sci Tech, Outre-Mer, Nouméa. Comment (DF): date agrees with type of burial and general data on human settlement of island.

\section{Iron Age}

\section{France}

\section{Ly-2780. Gypsum mine, Carcès, Var}

$2380 \pm 90$

$* 780-195 B C$

Wood from support timbers of gypsum mine $\left(43^{\circ} 29^{\prime} \mathrm{N}, 6^{\circ} 11^{\prime} \mathrm{E}\right)$. Coll 1980 by G Truc, Lab Geol, Univ Lyon I. Sample dated to check previous result, Ly-2223: $2570 \pm 130(\mathrm{R}, 1983, \mathrm{v} 25, \mathrm{p}$ 61). Comment: date is within statistical margin of previous one, and average of both measurements is $2440 \pm 70$.

\section{Derrière le Moulin series, Mours, Val d'Oise}

Samples from two levels of ditch found in quarry of Mafa Cie $\left(49^{\circ} 8^{\prime} \mathrm{N}\right.$, $\left.2^{\circ} 16^{\prime} \mathrm{E}\right)$. Coll and subm 1982 by JL Brulé, Cergy.

General Comment (JLB): as expected, boundary between La Tène II and III is dated at ca 2200 BP. Ly-2976 shows that charcoal from Layer VII may have descended into Layer V, below. 
TABLE 27

Derrière le Moulin

\begin{tabular}{rcclccc}
\hline $\begin{array}{c}\text { Sample } \\
\text { no. }\end{array}$ & Layer & $\begin{array}{c}\text { Depth } \\
(\mathrm{m})\end{array}$ & Expected period & DR & $\begin{array}{c}\text { Age } \\
(\mathrm{BP})\end{array}$ & $\begin{array}{c}\text { Corrected date } \\
\text { interval }\end{array}$ \\
\hline Ly-2977 & VII & 1 & La Tène III & 0.7 & $2120 \pm 120$ & $400 \mathrm{BC}-\mathrm{AD} 40$ \\
-2976 & V-AB & 2 & La Tène II \& III & 1 & $2150 \pm 110$ & $405 \mathrm{BC}-\mathrm{AD} 25$ \\
-2975 & V-AB & 2.5 & La Tène II \& III & 1 & $2320 \pm 110$ & $760 \mathrm{BC}-\mathrm{AD} 170$ \\
\hline
\end{tabular}

\section{La Pierre d'Appel series, Etival-Clairefontaine, Vosges}

Samples from two excavation areas in fortified promontory at top of plateau ( $\left.48^{\circ} 23^{\prime} \mathrm{N}, 6^{\circ} 51^{\prime} \mathrm{E}\right)$. Coll 1974 from $\mathrm{W}$ excavation, inside fortified habitat and 1981 from SW excavation in oldest fortification rampart, subm 1978 and 1982 by A Deyber, Herpiacum Soc, Etival-Clairefontaine (Deyber et al, 1984).

General Comment (AD): Ly-1731, from charred beam, does not come from archaeol level. Ly-2705 and -2704 agree with expected age, La Tène II. Ly-1732 is older by ca $300 \mathrm{yr}$, and comes from structure which is older than main habitation level.

TABLE 28

La Pierre d'Appel

\begin{tabular}{cccccc}
\hline $\begin{array}{c}\text { Sample } \\
\text { no. }\end{array}$ & $\begin{array}{c}\text { Ref, } \\
\text { colln } \\
\text { yr }\end{array}$ & Layer & Sample & $\begin{array}{c}\text { Age } \\
\text { (BP) }\end{array}$ & $\begin{array}{c}\text { Corrected } \\
\text { date } \\
\text { interval }\end{array}$ \\
\hline Ly-1731 & B-22, 1974 & Passage 5 & Charred beam & $690 \pm 140$ & AD1055-1425 \\
-1732 & B-22, 1974 & Passage 3 & Ash & $2530 \pm 130$ & $1015-390 \mathrm{BC}$ \\
-2705 & B-11, 1981 & Level 4g & Charcoal & $2220+100$ & $545-20 \mathrm{BC}$ \\
-2704 & B-11, 1981 & Level 4a & Charcoal & $2300 \pm 100$ & $755-165 \mathrm{BC}$ \\
\hline
\end{tabular}

Ly-2874. Caramontron, Sinzelle, Polignac, Haute Loire $\begin{array}{r}2340 \pm 150 \\ * 790-150 B C\end{array}$

Bones from ditch outcropping along roadside $\left(45^{\circ} 4^{\prime} \mathrm{N}, 3^{\circ} 52^{\prime} \mathrm{E}\right)$. Coll 1978 and subm 1982 by J Vital, Ampilhac-Vernassal. Sample coll as control for two previous measurements, Ly-2036: $2410 \pm 130$, and Ly-2037: $2520 \pm 120(\mathrm{R}, 1983, \mathrm{v} 25, \mathrm{p} 95)$, both estimated to be too young in relation to assoc industry from 1 st Iron age or Late Bronze age. Comment (JV): latest date agrees with two preceding ones, from Middle of Iron age. This may be due to continuation of Late Bronze and Early Iron age industries throughout Iron age (Houdré \& Vital, 1981).

Ly-2845. La Chauve-Souris cave, Donzère, Drôme $\quad \begin{array}{rr}\mathbf{2 5 2 0} \pm 140 \\ 010-390 B C\end{array}$

Charcoal from Layer $8 \mathrm{c} / 9$ of cave fill $\left(44^{\circ} 28^{\prime} \mathrm{N}, 4^{\circ} 41^{\prime} \mathrm{E}\right)$. Coll and subm 1982 by J Vital. Assoc with industry from beginning of Iron age (Vital, 1981). Comment (JV): date probably indicates Middle of Iron age, but remains compatible with expected age if max statistical margin is considered. 
En Bizier series, Jugy, Saône et Loire

Samples from 10 to $20 \mathrm{~cm}$ depth in hearth structure of only habitation level ( $\left.46^{\circ} 37^{\prime} \mathrm{N}, 4^{\circ} 52^{\prime} \mathrm{E}\right)$. Coll 1967 and subm 1983 by A Jeannet, Charnaylès-Macon.

Ly-3053. En Bizier 1

Charcoal $(0.5 \mathrm{dr})$.

Ly-3054. En Bizier 2

Bones (Ovicapridae).

General Comment (AJ): dates agree perfectly and are within expected range of assoc Early Iron age industry assumed to come from W Germany or N Switzerland (Jeannet, 1981).

\section{Ly-2529. La Prée tumulus, Thury, Côte d'Or}

$2300 \pm 220$

$* 800 B C-A D 20$

Charcoal from beneath endmost upright flagstone of sepulture of Tumulus III $\left(47^{\circ} 2^{\prime} \mathrm{N}, 4^{\circ} 30^{\prime} \mathrm{E}\right)$. Coll 1980 and subm 1981 by JP Guillaumet, Centre Natl Recherche Sci, Autun $(0.7 \mathrm{dr})$; assoc with Middle Hallstatt industry. Comment (FPG): date seems too young, perhaps due to wide statistical margin (Guillaumet \& Maranski, 1982).

Ly-2678. Ravin de Mardou, La Roche-Blanche, Puy de Dôme

$$
2590 \pm 120
$$

$* 1010-425 B C$

Bones (Ovicapridae) from ditch atop colluvial deposit $\left(45^{\circ} 43^{\prime} \mathrm{N}\right.$, $3^{\circ} 9^{\prime}$ E). Coll 1980 and subm 1982 by JP Daugas and JP Raynal, assoc with painted ceramics attributed to beginning of Early Iron age. Comment (JPD \& JPDR): date agrees with archaeol data (Daugas, Gilbert, \& Raynal, 1982) and with measurements obtained from another ditch site, Caramontron (above).

\section{Bronze Age}

France

\section{Ly-2698. Champ d'Ile, Assenay, Aube}

$2450 \pm 140$

$* 830-195 B C$

Charcoal from $1.25 \mathrm{~m}$ depth in open-air site $\left(48^{\circ} 11^{\prime} \mathrm{N}, 4^{\circ} 3^{\prime} \mathrm{E}\right)$. Coll 1980 and subm 1981 by JL Coudrot, Troyes $(0.3 \mathrm{dr})$. Assoc with Late Bronze age industry corresponding to end of "Champ d'Urnes" period and assumed to date from ca $700 \mathrm{BC}$ (Chertier, 1981). Comment (JLC): date is too young by ca 200 yr but suggests continuation of "Champ d'Urnes" civilization until beginning of Iron age. 


\section{Marais de Saint-Clair series, Marchezieux, Manche}

Wood from marsh sediments $\left(49^{\circ} 10^{\prime} \mathrm{N}, 1^{\circ} 18^{\prime} \mathrm{W}\right)$ in which eight probably contemporaneous caches of bronze axes "à douille" were found (Verron \& Tabbagh, 1983).

\section{Ly-2813. Cache F}

$1430 \pm 150$

From stake near Cache F.

\section{Ly-2676. Cache B}

$2470 \pm 120$

From tree branch placed over Cache B, probably to conceal it.

General Comment (GV): Ly-2813 shows stake had no connection with axe caches. Ly-2676 confirms that caches are one of last manifestations of Bronze age, which is contemporaneous with 1st Iron age.

\section{Gué des Plies series, Châlon-sur-Saône, Saône et Loire}

Samples from Bronze age habitation excavated from present river bed of La Saône R ( $\left.46^{\circ} 48^{\prime} \mathrm{N}, 4^{\circ} 50^{\prime} \mathrm{E}\right)$. Coll and subm 1982 by L Bonnamour, Mus Denon, Châlon-sur-Saône.

\section{Ly-2746. Châlon-sur-Saône, No. 2}

$2600 \pm 130$

*1085-410BC

Wood from upper part of archaeol level, corresponding to site destruction.

\section{Ly-2745. Châlon-sur-Saône, No. 1}

$2860 \pm 130$

*1400-790BC

Charred branches from middle of archaeol level, assoc with rich Late Bronze age IIIb industry.

General Comment (LB): both dates are within expected range (Bonnamour, 1983). However, Ly-2746 may be slightly later than actual site abandonment.

\section{Chabris Island series, Indre}

Charcoal from several areas and depths of open-air site comprising ca $2 \mathrm{~m}$ ashy layers in sandy alluvia of Le Cher $\mathrm{R}\left(47^{\circ} 17^{\prime} \mathrm{N}, 1^{\circ} 37^{\prime} \mathrm{E}^{\circ}\right)$. Coll 1981 and subm 1983 by J Despriés, Dir Antiquités Prehist, Orléans. Archeol level contains flint and ceramic artifacts attributed to Late Bronze age, as well as two deposits of bronze artifacts (Allain, 1981).

\section{Ly-3040. Chabris D3 425}

From $173 \mathrm{~cm}$ depth, Sq D3 (0.5 dr).

$$
* 1355-630 B C
$$




\section{Ly-3041. Chabris D5 246}

From $165 \mathrm{~cm}$ depth, Sq D5 (0.7 dr).

\section{Ly-3042. Chabris E3 173}

$* 2165-1360 B C$

$2790 \pm 150$

*1355-630BC

General Comment ( JD): Ly-3040 and -3042 completely agree with expected age of assoc industry. Ly-3041 could indicate that site had already been occupied during Middle Bronze age or beginning of Late Bronze age.

\section{Mont Sainte-Odile series, Ottrott, Bas-Rhin}

Charcoal from beneath and between foundation stones of long megalithic wall, "Le Mur Païen" (48 26' N, 7²4' E). Coll 1966 in excavation Site A and subm by $\mathrm{H}$ Zumstein, Archéol Mus, Strasbourg. Very diluted samples despite large size, due to solubility at alkaline pretreatment, caused by long exposure to forest soil.

\section{Ly-2800. Mont Sainte-Odile A}

$4400 \pm 400$

From $130 \mathrm{~cm}$ depth, between stones $(0.1 \mathrm{dr})$, subm 1982.

\section{Ly-2801. Mont Sainte-Odile B}

$3200 \pm 120$

From 130cm depth, 80cm from Ly-2800 (0.5 dr), subm 1982.

\section{Ly-2927. Mont Sainte-Odile 9.3-9.6}

From under wall ( $0.8 \mathrm{dr})$, subm 1983.

General Comment (HZ): statistical margin of Ly-2800 is too large; sample is obviously too old and has no connection with wall. As archaeol data suggest, wall was constructed on Late Bronze age site, with which Ly-2801 fits, either at end of this period, ca $1000 \mathrm{BC}$, or at beginning of Iron age (Ly-2927).

\section{Ly-2812. La Baume Layrou, Trèves, Gard}

$3520 \pm 110$

*2160-1670BC

Wood from top of fill in Sec C of cave $\left(44^{\circ} 5^{\prime} \mathrm{N}, 3^{\circ} 24^{\prime} \mathrm{E}\right)$. Coll and subm 1982 by L Fagès, Florac. Two occupations took place in cave, during Late Neolithic and mainly during Late Bronze age, from evidence of numerous potsherds (Fagès, 1982). Comment (GF): date confirms soil of cave remained unmodified since Middle Bronze age or very beginning of Late Bronze age.

\section{Lescar and Lons Tumuli series, Pyrénées Atlantiques}

Charcoal from hillock with several tumuli assumed to be from Bronze age (Blanc, 1982) $\left(43^{\circ} 20^{\prime} \mathrm{N}, 0^{\circ} 24^{\prime} \mathrm{W}\right)$, subm 1982 by C Blanc, Pau. 


\section{Ly-2707. Tumulus T1, Lescar}

$3650 \pm 150$ dr). Coll 1982. No assoc industry;

\section{Ly-2708. Tumulus T6, Lons}

$4260 \pm 150$

From $35 \mathrm{~cm}$ depth in all sqs, $\mathrm{P}-\mathrm{Q} / 5-6$, of tumulus from $\mathrm{S}$ part of hillock. Coll 1980 (0.6 dr). Assoc with pebble structure with potsherds.

\section{Ly-2709. Tumulus T5, Lons}

$3510 \pm 100$

From $57 \mathrm{~cm}$ depth, in Zone R-S/7-8, pit near Tumulus 5 in NE part of hillock. Coll 1982.

General Comment (CB): dates from Tumuli $\mathrm{T} 1$ and $\mathrm{T} 5$ are as expected. Date from Tumulus $\mathrm{T} 6$ seems too old although possible as archaeol data are same as for other 2 tumuli; 3 dates are comparable to unpub results from Tumulus BBL I of Boueilh (Gif-5525: $3620 \pm 120$ ) and from Tumulus T II of Lescar (Ny-250: $3959 \pm 70)$, unpub.

\section{Ly-2641. Le Grand Marais, Bucy le Long, Aisne}

$3710 \pm 200$

*2640-1690BC

Charcoal from $80 \mathrm{~cm}$ depth at base of pit with cremated remains in funerary urn $\left(49^{\circ} 23^{\prime} \mathrm{N}, 3^{\circ} 25^{\prime} \mathrm{E}\right)$. Coll 1980 and subm 1982 by C Pommepuy, Dir Antiquités Prehist, Amiens $(0.2 \mathrm{dr})$. Pit is part of funerary circle from Early or Middle Bronze age (Pommepuy \& Brun, 1984). Comment (CP): despite wide margin, date adequately confirms archaeol attribution of remains to Early Bronze age. This result cannot be compared with any others because cremation remains are rarely preserved in this type of site.

\section{Ly-2734. Chaleil, Saint-Cernin-de-Larche, Corrèze}

Human bones from sepulchral room of dolmen $\left(45^{\circ} 6^{\prime} \mathrm{N}, 1^{\circ} 24^{\prime} \mathrm{E}\right)$. Coll 1981 and subm 1982 by G Mazière, Dir Antiquités Prehist, Limoges. Assoc with remains of pearls and pendants (Mazière, 1983). Comment (GM): date indicates beginning of Bronze age and fits well with dates from similar monuments nearby in Quercy prov, such as La Bertrandoune dolmen at Prayssac, Ly-1220: $4170 \pm 120(\mathrm{R}, 1978, \mathrm{v} 20, \mathrm{p} 41)$, Les Grèzes dolmen at Souillac, Ly-895: $3910 \pm 120(\mathrm{R}, 1976, \mathrm{v} 18, \mathrm{p} 73)$, and Le Riffat dolmen at Thédirac, Ly-1188: $4090 \pm 130(\mathrm{R}, 1978$, v 20, p 41).

\section{Switzerland}

\section{Ly-2922. Horn, Wittnaü, Basel Canton}

$\mathbf{2 6 8 0} \pm \mathbf{1 7 0}$

*1275-410BC

Bones of animal spp from hearth, ca $1 m$ depth, in fortified promontory site $\left(47^{\circ} 28^{\prime} \mathrm{N}, 7^{\circ} 7^{\prime} \mathrm{E}\right)$. Coll 1982 by P Gutzwiller and subm 1982 by LR Berger, Univ Basel ( $0.5 \mathrm{dr})$. Assoc with ceramic industry from Late Bronze 
age IIIb (Gassler, 1982). Comment (LRB): date agrees with chronol data from region, which show Iron age began at ca $700 \mathrm{BC}$. It also confirms dendrochronol dates from Zurich lab.

\section{Spain}

\section{S Spain sites series}

Samples coll and subm by F Molina, Prehist Dept, Univ Granada.

General Comment (FM): all results agree with expected values and confirm several dates from Groningen lab from other sites in region with same assoc industry. These results suggest relatively late date for end of Copper age (Ly-2653) and date comparable with Middle Bronze age, French equivalent of Spanish Argar B.

TABLE 29a

Spanish sites

\begin{tabular}{cccccc}
\hline Site & Ref & Village & Province & Geog coordinates & $\begin{array}{c}\text { Colln } \\
\text { yr }\end{array}$ \\
\hline $\begin{array}{c}\text { Cerros de los } \\
\begin{array}{c}\text { Castellones } \\
\text { Motilla des } \\
\text { Azuer }\end{array}\end{array}$ & $(\mathrm{C} \mathrm{d} \mathrm{C})$ & Laborcillas & Granada & $\left(37^{\circ} 27^{\prime} \mathrm{N}, 3^{\circ} 17^{\prime} \mathrm{W}\right)$ & 1973 \\
$\begin{array}{c}\text { Cerro de la } \\
\text { Encina }\end{array}$ & $(\mathrm{C} \mathrm{d} \mathrm{E})$ & Daimiel & Ciudad Real & $\left(39^{\circ} 03^{\prime} \mathrm{N}, 3^{\circ} 30^{\prime} \mathrm{W}\right)$ & 1976 \\
\hline
\end{tabular}

TABLE 29b

Spanish sites

\begin{tabular}{|c|c|c|c|c|c|c|c|}
\hline $\begin{array}{c}\text { Sample } \\
\text { no. }\end{array}$ & $\begin{array}{l}\text { Site } \\
\text { ref }\end{array}$ & $\begin{array}{c}\text { Sample } \\
\text { ref }\end{array}$ & Sample & $\begin{array}{c}\text { Depth } \\
\text { (m) }\end{array}$ & $\begin{array}{c}\text { Assoc } \\
\text { industry }\end{array}$ & $\begin{array}{l}\text { Age } \\
\text { (BP) }\end{array}$ & $\begin{array}{l}\text { Corrected } \\
\text { date } \\
\text { interval } \\
\text { (BC) }\end{array}$ \\
\hline Ly-2653 & $\mathrm{CdC}$ & CB5-5125 & Charred seed & $3-1.4$ & Late Copper & $3500 \pm 130$ & $2280-1560$ \\
\hline-2654 & $\mathrm{Md} \mathrm{A}$ & D6-6016 & Charred seed & $1-1.2$ & Middle Bronze & $3530 \pm 130$ & $2300-1585$ \\
\hline-2655 & $\mathrm{M} \mathrm{d} \mathrm{A}$ & Dl -327 & Charred seed & $0.8-0.9$ & Middle Bronze & $3540 \pm 130$ & $2305-1640$ \\
\hline-2656 & $\mathrm{CdE}$ & M7-21177 & Charcoal & ca 2.8 & Middle Bronze & $3350 \pm 100$ & $1900-1425$ \\
\hline-2657 & $\mathrm{CdE}$ & M17-16053 & Charcoal & ca 2.6 & Middle Bronze & $3520 \pm 110$ & $2160-1670$ \\
\hline
\end{tabular}

Chalcolithic/Late Neolithic Periods

\section{France}

\section{Ly-2964. Fond Pernant, Compiègne, Oise}

$\mathbf{3 8 9 0} \pm \mathbf{1 8 0}$

*2890-1900BC

Bones from $60 \mathrm{~cm}$ depth from Ditch 28 of open-air site habitation area $\left(49^{\circ} 24^{\prime} \mathrm{N}, 2^{\circ} 48^{\prime} \mathrm{E}\right)$. Coll 1982 by B Lambot and subm 1982 by JC Blanchet, Centre Recherche Archéol Oise, Compiègne. Assoc with Chalcolithic industry of "Groupe des Urnes" type, just before Early Bronze age Atlantic type. Comment (JCB): date agrees perfectly with age expected for this type of industry in N France (Blanchet, 1983). 
Ly-2688. Broum cave, Hérault

Charcoal from hearth in copper cave mine $\left(43^{\circ} 35^{\prime} \mathrm{N}, 2^{\circ} 48^{\prime} \mathrm{E}\right)$. Coll and subm 1981 by P Ambert, Lab Geog, Univ Aix-Marseille; assoc with Late Neolithic industry of Ferrières type. This site may be oldest copper mine in region (Vasseur, 1911). Comment (PA): despite hearth's strat context, date shows it is not part of archaeol level. Another hearth in site analyzed by Gif lab yielded date in expected age range, Gif-6048: $3990 \pm 70$ (unpub).

\section{Ly-2954. Chomérac cave, Chomérac, Ardèche}

$3940 \pm 160$

*2880-2120BC

Human bones from fill of lower part of cave, embedded in recent level of calcareous breccia $\left(44^{\circ} 43^{\prime} \mathrm{N}, 4^{\circ} 39^{\prime} \mathrm{E}\right)$. Coll 1983 by B Aubert and subm 1983 by A Cogoluènhes, Lab Geol, Univ Lyon I ( $0.5 \mathrm{dr})$. Comment (AC): upper part of cave was used as communal grave during Neolithic, then was occupied during Wars of Religion (16th century). Date indicates fill preserved in lowest part of grave is Neolithic, in fact from Chalcolithic, as are many other such sepulchral caves in region.

\section{Boussargues series, Argelliers, Hérault}

Charred acorns from two areas in fortified habitation site $\left(43^{\circ} 44^{\prime} \mathrm{N}\right.$, $3^{\circ} 42^{\prime}$ E). Coll and subm 1982 by X Gutherz, Dir Antiquités, Montpellier. Site has only one habitation level, containing Chalcolithic artifacts of Fontbouïsse type.

\section{Ly-3016. Boussargues Loc 1}

$4170 \pm 140$

From Loc 1 near Hut 1 ( $0.7 \mathrm{dr})$.

\section{Ly-3017. Boussargues Cabane 1}

$\mathbf{4 0 7 0} \pm \mathbf{1 7 0}$

\section{From center of Hut 1.}

General Comment (XG): date agrees perfectly with others obtained for same cultural period and is comparable to dates from fortified sites from region such as Le Lébous at Saint-Martin-de-Tréviers, Hérault, as well as to dates from several unfortified villages, such as Campous at Viols en Laval, MC719: $3970 \pm 90$ (unpub).

\section{Claux cave series, Gourniès, Hérault}

Charcoal from 2 areas in cave $\left(43^{\circ} 51^{\prime} \mathrm{N}, 3^{\circ} 34^{\prime} \mathrm{E}\right)$. Coll 1981 and subm 1982 by JL Roudil, Dir Antiquités Prehist, Montpellier.

\section{Ly-2735. Claux, Zone C}

$4040 \pm 130$

From burial sepulture in superficial hearth, attributed to Chalcolithic of Fontbouïsse type. 


\section{Ly-2736. Claux, Zone $\mathrm{H}$}

From base of archaeol level at ca $15 \mathrm{~cm}$ depth corresponding to occupation attributed to Late Neolithic of Ferrières type $(0.3 \mathrm{dr})$.

General Comment (JRL): both values agree perfectly with generally accepted age range for assoc industries in both occupation levels of site; they also agree with result from Aven de Jacques (below).

\section{Aven de Jacques series, Chabessière, Lussas, Ardèche}

Human bones from two areas in cave near La Chabessière $\left(44^{\circ} 37^{\prime} \mathrm{N}\right.$, $5^{\circ} 21^{\prime} \mathrm{E}$ ). Coll 1981 and subm 1982 by A Héritier, Romans, and A Cogoluènhes. Cave was used for communal burial ground during Late Neolithic to Chalcolithic and until Late Bronze age, because it contains artifacts from these three cultural periods.

\section{Ly-2847. Aven de Jacques No. 2 \\ Ly-2846. Aven de Jacques No. 1 $(0.4 \mathrm{dr})$}

$3860 \pm 130$ $* 2730-2050 B C$

$4140 \pm 170$

*3245-2305BC

General Comment (AH): both dates agree with archaeol attribution to Chalcolithic period; Ly-2846 corresponds to beginning of period, which may be contemporaneous with Late Neolithic period of Ferrières type, and Ly2847 marks end of Chalcolithic period and may be contemporaneous with previous date from same site, Ly-2295: $3660 \pm 130(\mathrm{R}, 1983$, v 25, p 99). All three dates suggest rather lengthy use of cave as burial place, perhaps as late as Early Bronze age.

\section{Ly-2689. Roque-Fenètre, Cabrières, Hérault}

$3900 \pm 130$

*2815-2075BC

Charcoal from pit in copper mine $\left(43^{\circ} 35^{\prime} \mathrm{N}, 3^{\circ} 22^{\prime} \mathrm{E}\right)$. Coll and subm 1981 by P Ambert. Assoc with Late Neolithic industry of Verazian type. Comment (PA): date is within expected range and may be comparable to many other dates for Fontbouïsse and Verazian Chalcolithic or Late Neolithic industries in region (Gasco \& Binder, 1983).

\footnotetext{
$1890 \pm 180$

Ly-2571. La Pointe aux Oies, Wimereux, Pas de Calais * $380 B C-A D 545$

Human bones from communal burial in covered passage of long dolmen embedded in dunes on sea coast (50 $\left.47^{\prime} \mathrm{N}, 1^{\circ} 36^{\prime} \mathrm{E}\right)$. Coll 1979 and subm 1981 by JF Piningre, Dir Antiquités Prehist, Lille. No characteristic artifacts were assoc, but this type of megalithic monument is similar to those from Neolithic of "Seine-Oise-Marne" (SOM) type in Picardie region (Piningre, 1979). Comment (JFP): date shows burial occurred much later than construction of megalith, as reworked aspect of monument fill had suggested.
} 
Ly-3023. Villevieille, Demandolx, Alpes de Haute-Provence

Human bones from communal burial in dolmen chamber $\left(43^{\circ} 52^{\prime} \mathrm{N}\right.$, $6^{\circ} 35^{\prime}$ E). Coll 1974 and 1975 and subm 1983 by G Sauzade, Dir Antiquités Prehist, Aix en Provence. Assoc with Late Neolithic industry. Comment (GS): date is younger than expected and suggests site was in use for prolonged period; no archaeol evidence.

\section{Ly-2980. Cala Barbarina, Tizzano, Corse du Sud}

$4020 \pm 140$

Bones (Suidae) from complete skeleton found beside human burial in Layer III of Le Sanglier rock shelter $\left(41^{\circ} 31^{\prime} \mathrm{N}, 9^{\circ} 13^{\prime} \mathrm{E}\right)$. Coll 1980 by A Pasquet and subm 1983 by JD Vigne, Lab Anatomie Comparée, Natl Mus Hist Nat, Paris. Assoc with somewhat characteristic Early or Middle Neolithic industry. Comment (JDV): date is younger than expected and dates burial to Late Neolithic.

Ly-2658. Le Trou des Fées, Bayonville-sur-Mad, Meurthe et Moselle

$4210 \pm 170$

$* 3345-2415 B C$

Human bones from peculiar burial in sepulchral cave $\left(49^{\circ} 1^{\prime} \mathrm{N}\right.$, $5^{\circ} 58^{\prime}$ E). Coll 1977 and subm 1981 by C Guillaume, Dir Antiquités Prehist, Metz. Burials occurred in cave during two periods, Late Neolithic, corresponding to previous dates, Ly-1622: $4170 \pm 200$, and Ly-1623: $4280 \pm$ 150 (R, 1979, v 21, p 435), and Early Middle ages (ca 10th century). Skeleton lay at boundaries of both periods and was thought to be assoc with older level. Comment (CG): date is within statistical margins of both previous measurements and confirms attribution of burial to Neolithic.

\section{Ly-3021. Le Capitaine, Grillon, Vaucluse}

$4330 \pm 180$

*3360-2670BC

Human bones from collective sepulture below hypogeum $\left(44^{\circ} 24^{\prime} \mathrm{N}\right.$, $4^{\circ} 56^{\prime}$ E). Coll 1977 and 1978 by G Sauzade. Assoc with Late Neolithic industry. Comment (GS): date agrees perfectly with expected age and results obtained from lowest level of Roaix site, Gif-1620: $4100 \pm 140$ (R, 1971, v 13, p 219) (Sauzade, 1983).

$\mathbf{5 1 1 0} \pm \mathbf{2 5 0}$

Ly-2550. Chironlon or Fabrègues cave, Gras, Ardèche *4435-3370BC

Human bones from sepulchral ditch $\left(44^{\circ} 24^{\prime} \mathrm{N}, 4^{\circ} 38^{\prime} \mathrm{E}\right)$. Coll 1970 by A Héritier, Romans, and subm 1980 by A Cogoluènhes. Communal sepulture with ca 20 individuals and Chalcolithic industry of Fontbouïsse type. Comment (AH): date is much older than expected and does not correspond with archaeol data. As site does not contain proof of Middle Neolithic occupation, there may be error in origin of bones, due to long delay between colln and subm of sample. 


\section{Middle Neolithic Period}

France

\section{Cul-Froid series, Boury-en-Vexin, Oise}

Bones from enclosure ditch of habitation $\left(49^{\circ} 14^{\prime} \mathrm{N}, 1^{\circ} 3^{\prime} \mathrm{E}\right)$. Coll by $\mathrm{R}$ Martinez and subm 1982 by JC Blanchet.

\section{Ly-2712. Cul-Froid, No. 4}

$4570 \pm 130$

*3660-2995BC

From Layer D1 at top of fill, coll $1982(0.6 \mathrm{dr})$. Assoc with evolved Chassean industry.

\section{Lv-2711. Cul-Froid, No. 1}

$5180 \pm 100$

$* 4330-3690 B C$

From middle layer of fill, coll 1981. Assoc with Middle Chassean industry.

\section{Ly-2961. Cul-Froid, No. 3}

$\mathbf{5 2 4 0} \pm \mathbf{1 5 0}$

$* 4410-3690 B C$

From Layer G2 at base of fill, coll 1982 (0.5 dr). Assoc with Chassean industry with Roessen influence.

General Comment (JCB): three dates agree perfectly with strat and evolution of Chassean industry. They are comparable to dates from samples assoc with similar industries at Camp-de-César site and Le-Coq-Galleux site (below).

\section{Camp de César series, Catenoy, Oise}

Bones from several levels and sqs of excavation on edge of plateau of fortified promontory $\left(49^{\circ} 22^{\prime} \mathrm{N}, 2^{\circ} 31^{\prime} \mathrm{E}\right)$. Coll and subm by JC Blanchet (Blanchet \& Decormeille, 1984).

General Comment (JCB): dates agree perfectly with strat and evolution of industry. They compare well with 3 dates from Cul-Froid site (above) for same industries.

TABLE 30

Camp de César

\begin{tabular}{|c|c|c|c|c|c|c|}
\hline $\begin{array}{c}\text { Sample } \\
\text { no. }\end{array}$ & Layer & $\begin{array}{c}\text { Colln } \\
\mathrm{yr}\end{array}$ & Industry & DR & $\begin{array}{r}\text { Age } \\
(\mathrm{BP})\end{array}$ & $\begin{array}{c}\text { Corrected } \\
\text { date } \\
\text { interval } \\
\text { (BC) }\end{array}$ \\
\hline Ly-2967 & $5 a$ & 1982 & Late Chassean & 1 & $4550 \pm 160$ & $3655-2915$ \\
\hline-2965 & $5 b$ & 1982 & Late Chassean & 1 & $4620 \pm 120$ & $3655-3050$ \\
\hline-2968 & $5 b c$ & 1982 & Chassean & 0.7 & $4820 \pm 150$ & 3885-3355 \\
\hline-2713 & $5 c$ & 1981 & Middle Chassean & 1 & $4980 \pm 120$ & 3935-3565 \\
\hline-2966 & $5 d$ & 1982 & Middle Chassean & 0.5 & $5280 \pm 140$ & $4420-3785$ \\
\hline
\end{tabular}




\section{Ly-3020. Malvoisin, Orgon, Bouches-du-Rhône}

$5270 \pm 140$

$* 4420-3780 B C$

Bones from only occupation level of open-air site $\left(43^{\circ} 48^{\prime} \mathrm{N}, 5^{\circ} 1^{\prime} \mathrm{E}\right)$. Coll 1972 by A Carry and subm 1983 by G Sauzade; assoc with Chassean potsherds (Sauzade, 1983). Comment (GS): date fits well with expected age and numerous other dates from many Chassean sites in Provence region, particularly with nearby Claparouse site at Lagen, MC-1691: $5380 \pm 125$ (unpub).

\section{La Vergentière series, Cohons, Haute-Marne}

Charcoal from three excavation loci ( 2 borings and 1 hearth in dolmen) on fortified promontory $\left(47^{\circ} 46^{\prime} \mathrm{N}, 5^{\circ} 20^{\prime} \mathrm{E}\right)$. Coll and subm by $\mathrm{L}$ Lepage, Saint-Dizier. Test excavation of archaeol levels reveal Middle Neolithic industry of Bourgogne type, whereas dolmen has no assoc industry (Lepage, 1980).

Ly-2646. La Vergentière S1-R18-117

$5110 \pm 130$

From upper part of Level D in Boring S1; coll and subm 1981.

\section{Ly-2647. La Vergentière S2-A7-150}

$\mathbf{5 2 7 0} \pm \mathbf{3 9 0}$

From lowest level of Boring S2; coll and subm 1981 (0.1 dr).

\section{Ly-3055. La Vergentière, Dolmen 1}

$\mathbf{5 3 0 0} \pm \mathbf{2 6 0}$

$* 4690-3645 B C$

From hearth in tumulus of Dolmen 1; coll and subm 1982 (0.2 dr).

General Comment (LL): despite wide statistical margin for Ly-2647 and $-3055,3$ dates are quite similar to previous results from Boring 1, Ly-1859: $5230 \pm 300$ and Ly-1860: $5350 \pm 270(\mathrm{R}, 1983, \mathrm{v} 25, \mathrm{p} 105)$. They suggest relatively short occupation of site and contemporaneity of dolmen with habitation level excavated in borings (Lepage, 1982).

\section{Roquefort series, Lugasson, Gironde}

Bones from two levels of fortified promontory $\left(44^{\circ} 44^{\prime} \mathrm{N}, 0^{\circ} 9^{\prime} \mathrm{W}\right)$ coll from 1973 to 1976 and subm 1981 by J Roussot-Larroque, Lab Quaternary Geol, Univ Bordeaux I. Site has two parts, Le Plateau and Le Talus. Archaeol levels contain complete sequence from Middle Neolithic of Roquefort group to Late Neolithic of Matigon and Artenacian types.

\section{Ly-2683. Roquefort-Plateau, Layer 2}

$3650 \pm 190$ $* 2540-1665 B C$

From Layer 2, Plateau site; assoc with Matignon Late Neolithic industry $(0.25 \mathrm{dr})$. 


\section{Ly-2684. Roquefort-Plateau, Layer 3}

$\mathbf{5 6 8 0} \pm \mathbf{2 0 0}$

*5000-4110BC

From Layer 3, Plateau site, assoc with ist phase of Roquefort Middle Neolithic $(0.2 \mathrm{dr})$.

General Comment (JRL): Ly-2683 is obviously too young for Neolithic industry. Such aberrant results were already observed for charcoal from same level, Gif-3597: $3960 \pm 135$ (unpub). Ly-2684 agrees perfectly with other results from both phases of Roquefort group in Talus site, Gif-1732: $5000 \pm 140$ for Phase 2, and Gif-1731: $4800 \pm 140$ for Phase 1 (RoussotLarroque, 1976).

\section{Ly-2613. Le Trou qui fume, Saint-Romain, Côte d'Or $* 4560-3905_{B C}$}

Bones (Bovidue) from top of fili of small cave $\left(47^{\circ} 0^{\prime} \mathrm{N}, 4^{\circ} 43^{\prime} \mathrm{E}\right)$. Coll 1981 by PY Jacquet and subm 1981 by $s$ Grappin, Dijon. Cave was used only as refuse pit. Comment (SG): date confirms homogeneity of fill and end of use of pit in Middle Neolithic (Grappin, 1982).

\section{Ly.2970. Le Mont d'Huette, Jonquières, Oise}

$5300 \pm 140$

$* 4425-3790 \mathrm{BC}$

Bones from base of palisade ditch of fortified habitation $\left(49^{\circ} 24^{\prime} \mathrm{N}\right.$, $2^{\circ} 44^{\prime} \mathrm{E}$ ). Coll and subm 1982 by JC Blanchet. Sample comes from Sec 1th, with Early Chassean industry of Paris basin type. Comment (JCB): date agrees with expected age and dates from other sites in region with Early Chassean industries (see, eg, Ly-2996 and -2961, above). Three other measurements were made for same site: Gif-1623: $1600 \pm 300(\mathrm{R}, 1974$, v 16, p 38) and Gif-2918:4290 \pm 100 (unpub) are obviously too young and are from charcoal which may have been polluted by roots; Gif-2929: $5120 \pm$ 130 (unpub) was on bones from same ditch and also fits witl same industry as Ly-2970, Early Chassean of Fpi-Rocssen type of Menneville.

\section{Le Verger series, Saint-Romain, Côte dor}

Charcoal from Layer 2 of site at foot of cliff $\left(46^{\circ} 59^{\prime} \mathrm{N}, 4^{\circ} 43^{\prime} \mathrm{E}\right)$. Coll and subm 1982 by $\mathrm{S}$ Grappin. Assoc with industry attributed to beginning of Middle Neolithic (Grappin, 1982$)(0.3$ and $0.8 \mathrm{dr}$, respectively).

\section{Ly-2706. Le Verger No. A}

$5260 \pm 180$

$* 4435-3670 B C$

\section{Ly-2971. Le Verger No. 5}

$5710 \pm 140$

$* 4945-4370 B C$

General Comment $(\mathrm{SQ})$. both dates confrim two previous results from samples from sanie archaeol level, Ly-1985:5590 \pm 130 and Ly-2243:5860 \pm $170(\mathrm{R}, 1983, \mathrm{v} 25, \mathrm{p} 105)$ These dates have same statistical margin and their average is $5620 \pm 80 \mathrm{BP}$. This confirms, as presumed, that this type of industry precedes Middle Neolithic Chassean in region (sec Chassey series, $R, 1983, v 25, p 103$ ) and is contemporaneous with industry from Chichery 
site, Yonne, Gif-3354: $5600 \pm 120$ (unpub) and with Middle Neolithic industries of Cerny type.

\section{Montagne de Comin series, Bourg-et-Comin, Aisne}

Charcoal from burned layer assoc with earth mound in open-air fortified habitation atop plateau $\left(49^{\circ} 25^{\prime} \mathrm{N}, 3^{\circ} 40^{\prime} \mathrm{E}\right)$. Coll and subm by Unité Recherche No. 12 Centre de Recherche Archéol, Paris.

Ly-2972. BMC No. 6

$\mathbf{5 4 4 0} \pm 160$

$* 4550-3890 B C$ 1982.

From $1.8 \mathrm{~m}$ depth underlying earth mound $(0.5 \mathrm{dr})$; coll and subm

Ly-2973. BMC No. 7

$4640 \pm 220$

From $1 \mathrm{~m}$ depth at base of earth mound $(0.3 \mathrm{dr})$; coll and subm 1982.

Ly-3052. BMC No. 9

$\mathbf{5 8 7 0} \pm \mathbf{1 6 0}$

*5195-4435BC

From $1.7 \mathrm{~m}$ depth at base of earth mound above ditch of palisade $(0.5$ dr); coll and subm 1983.

General Comment (URA No. 12): three dates, as well as Ly-2525: $4880 \pm 120$ $(\mathrm{R}, 1983, \mathrm{v} 25, \mathrm{p} 102)$ from $1.2 \mathrm{~m}$ depth in same layer prove that earth mound dates from prehist period, despite uncertainty of results of strat study. Dates show that burned layer is assoc with oldest occupation period of site, contemporaneous with Middle Neolithic of Michelsberg type industry, previously dated to ca $5100 \mathrm{BP}$, eg, in two sites in Aisne R valley ( $R$, 1983 , v 25, p 106). However, Ly-3051 seems too old even if double statistical margin is used.

\section{Clesmp de Bataille series, L'Etoile, Somme}

Charcoal from open-air habitation surrounded by palisade $\left(50^{\circ} 2^{\prime} \mathrm{N}\right.$, $2^{\circ} 39^{\prime} \mathrm{E}$ ). Coll and subm by B Bréart, Dir Antiquités Préhist, Amiens. Ditches contain fairly sparse industry attributed to beginning of Middle Neolithic, perhaps of "Cerny" type.

\section{Ly-2679. Champ de Bataille No. 1}

$5390 \pm 100$

From base of post hole of palisade; coll and subm 1981

Ly-3058. Champ de Bataille No. 2

$5990 \pm 160$

*5255-4555BC

From base of ditch assoc with palisade $(0.3 \mathrm{dr})$. Coll and subm 1983.

General Comment (BB): Ly-2679 agrees with attribution of industry to Middle Neolithic; Ly-3058 is comparable to many dates for Early Neolithic of late Rubané type, and suggests relatively old date for Cerny group, even taking into account double statistical margin. 


\section{Early Neolithic Period}

France

\section{Ly-2969. Le Jocoy, Pontpoint, Oise}

$5380 \pm 220$

*4555-3795BC

Bones from middle of Ditch 1 in river-bank site in Oise $\mathrm{R}$ alluvia $\left(49^{\circ}\right.$ $20^{\prime} \mathrm{N}, 2^{\circ} 39^{\prime} \mathrm{E}$ ) (Blanchet, Decormeille, \& Marquis, 1980). Coll 1982 by A Decormeille and subm 1982 by JC Blanchet. Assoc with Early Neolithic industry of Late Rubané type $(0.25 \mathrm{dr})$. Comment (JCB): for unknown reason, date is much too young, by ca $500 \mathrm{yr}$, as compared, eg, to Le-CoqGalleux and Cuiry-Les-Chaudardes sites (below).

\section{Les Fontinettes series, Cuiry-les-Chaudardes, Aisne}

Bones from two refuse pits in Neolithic village $\left(49^{\circ} 23^{\prime} \mathrm{N}, 3^{\circ} 45^{\prime} \mathrm{E}\right)$. Coll and subm 1981 by Unité de Recherche No. 12, Paris, to complement previously pub series of 13 samples assoc with Early Neolithic Late Rubané type industry (R, 1983, v 25, p 106).

\section{Ly-2551. CCF No. 378-2}

$5870 \pm 170$

Coll 1980 (0.8 dr).

\section{Ly-2552. CGF No. 382}

$5730 \pm 170$

Coll 1981 ( $0.9 \mathrm{dr})$.

General Comment (URA No. 12): both dates agree perfectly with all previously pub dates. Bell-shaped curve drawn from 15 results indicates that occupation responsible for this industry was very short, $i e, 200 \mathrm{yr}$, and occurred ca 5950 BP (Constantin \& Lasserre, 1983). Assoc with Cardial Early $\mathrm{N}$ (JRL): date is a little younger than other dates from shoreline sites on $\mathrm{W}$ French coast, eg, at Les Gouillauds, on Ré I., Gif-4878: $5950 \pm 100$ (Pautreau \& Robert, 1980) and at La-Tranche-sur-Mer, Vendée, Gif-4372: $6300 \pm 160$ (Jousseaume et al, 1979). Both sites also contain Early Neolithic industry, but Balise site shows Middle Neolithic attributes (RoussotLarroque, 1976) and, thus, may be younger.

\section{Ly-2651. Breisberg, Oudrenne, Moselle}

$5990 \pm 200$ quités Prehist, 
linear Rubané type ( $0.7 \mathrm{dr})$. Comment (CG): date agrees with expected value and fits exactly with one from Kirchnaumen, nearby site with same industry, Ly-1181: $6060 \pm 200(\mathrm{R}, 1978, \mathrm{v} 20, \mathrm{p} 43)$ (Decker, Guillaume, \& Michels, 1977). Thus, as expected, it is also a little younger than Late Rubané sites in neighboring regions but similar to Paris basin sites such as Cuiry-Les-Chaudardes (above) and Le-Coq-Galleux (below).

\section{Le Rochas series, Saint-Remèze, Ardèche}

Charcoal from three superimposed sub-levels in Layer 4 of cave fill $\left(44^{\circ} 21^{\prime} \mathrm{N}, 4^{\circ} 29^{\prime} \mathrm{E}\right)$. Coll 1981 and subm 1982 by A Beeching, Centre Natl Recherche Sci, Marseille. Layer 4 contains homogeneous Middle or Late Cardial Early Neolithic industry.

\section{Ly-2749. Rochas 4-1/4-2}

$5060 \pm 250$

Samples from upper sub-levels $4-1$ and $4-2$; only $0.2 \mathrm{dr}$ despite avaitability of ca $20 \mathrm{~g}$ of charcoal before alkaline pretreatment.

\section{Ly-2748. Rochas 4-3}

$6090 \pm 210$

From lowest sub-level 4-3; only $0.4 \mathrm{dr}$ also after large dissolution of charcoal during alkaline pretreatment.

General Comment (AB): Ly-2748 agrees with expected age for Late Cardial Neolithic and previously obtained results from region, eg, in nearby site, $\mathrm{La}$ Baume-Bourbon at Cabrières, Ly-412: $6050 \pm 120$ and Ly-538: $6180 \pm$ $180(\mathrm{R}, 1973$, v 15, p 525-526) or in La Baume rock shelter at Montclus, Ly-303/304: $6220 \pm 100(\mathrm{R}, 1971, \mathrm{v} 13, \mathrm{p} 62)$. Ly-2749 is obviously too young and may be explained by presence of some charcoal originating from overlying layers which contain industries dating from Chalcolithic to Middle Ages, despite presence of stalagmitic floor overlying Layer 4. Discrepancy may also be due to large statistical margin caused by small dr (Beeching \& Thomas-Beeching, 1982).

\section{Rufacher Huben series, Colmar, Haut-Rhin}

Bones from several refuse pits of village ( $\left.48^{\circ} 2^{\prime} \mathrm{N}, 7^{\circ} 20^{\prime} \mathrm{E}\right)$. Coll 1979 by $\mathrm{C}$ Bonnet and subm 1982 by A Thévenin, Dir Antiquités Prehist, Stras-

TABLE 31

Rufacher Huben

\begin{tabular}{clccc}
\hline $\begin{array}{c}\text { Sample } \\
\text { no. }\end{array}$ & Site ref & DR & $\begin{array}{c}\text { Age } \\
(\mathrm{BP})\end{array}$ & $\begin{array}{c}\text { Corrected } \\
\text { date } \\
\text { interval } \\
\text { (BC) }\end{array}$ \\
\hline Ly-2729 & RST 13-14 & 0.4 & $5990 \pm 230$ & $5285-4450$ \\
-2730 & MN 2 & 0.5 & $5860 \pm 140$ & $5190-4430$ \\
-2731 & HI 13-15 & 0.4 & $5690 \pm 180$ & $5020-4115$ \\
-2732 & DE 18-20 & 0.5 & $6050 \pm 200$ & $5325-4550$ \\
-2733 & CD 13-14-15 & 1 & $5740 \pm 130$ & $4990-4395$ \\
\hline
\end{tabular}


bourg. Assoc with Rubané artifacts. Each pit contains unstrat mixture of bones and potsherds from Early, Middle, and Late Rubané.

General Comment (CB): despite dilution of samples due to low collagen content of most of bones, as is usual for open-air sites, dates are very similar for all pits. This suggests either homogeneous mixture of bones or very short site occupation which was contemporaneous with Late Rubané in Lorraine prov, eg, in Breisberg site (above), or in Paris basin, eg, in Cuiry-les-Chaudardes site (above). Brevity of occupation does not agree with typol data from potsherds mixed in ditches, of which most are Early Rubané and surely older than $6000 \mathrm{BP}$. Thus, due to lack of strat in ditches, results do not actually date whole site, but demonstrate homogeneity of bone mixture.

\section{Le Coq Galleux series, Compiègne, Oise}

Bones from habitation site on lowest terrace of Oise $\mathrm{R}\left(49^{\circ} 24^{\prime} \mathrm{N}\right.$, $2^{\circ} 48^{\prime} \mathrm{E}$ ). Coll 1978 (Ly-2714 and -2715 ) by C Toupet and 1979 by JC Blanchet and subm 1982 by JC Blanchet.

General Comment (JCB): except for Ly-2715, which was sampled at very shallow depth and may, thus, be younger, all dates are perfectly within expected range indicated by strat study and typol of assoc industries (Toupet, 1980; Blanchet \& Decormeille, 1980). First phase of site occupation occurred during Early Neolithic (Paris basis Rubané type). Three dates from this period (Ly-2717, 2716, -2720) are very close and similar to those obtained for same industry in many other sites of region, such as CuiryLes-Chaudardes (above; R, 1983, v 25, p 106). Second phase of site occupation corresponds to Cerny type Middle Neolithic; both dates from this period agree with similar samples from Paris basin and N France, such as Les Sablins at Etaples, Gif-3701: $5660 \pm 120$ and Gif-4024: $5690 \pm 120$

TABLF 32

Le Coq Galleux

\begin{tabular}{|c|c|c|c|c|c|}
\hline $\begin{array}{l}\text { Sample } \\
\text { no. }\end{array}$ & Loc in site & $\begin{array}{l}\text { Assoc } \\
\text { industry }\end{array}$ & DR & $\begin{array}{l}\text { Age } \\
(\mathrm{BP})\end{array}$ & $\begin{array}{l}\text { Corrected } \\
\text { date } \\
\text { interval } \\
\text { (BC) }\end{array}$ \\
\hline L.y-2962 & Struct 12, pit & $\begin{array}{l}\text { L.ate Neolithic } \\
\text { Chalcolithic }\end{array}$ & 0.3 & $3870 \pm 130$ & $2785-1970$ \\
\hline-2715 & Pit 2 & Chassean & 1 & $4450 \pm 140$ & $3315-2870$ \\
\hline-2714 & Level 2 Sec 3 & Chassean & 1 & $4950 \pm 160$ & $4075-3880$ \\
\hline-2718 & $\begin{array}{l}\text { Struct } 14 \\
\text { Camp ditch }\end{array}$ & Early Chassean & 1 & $5330 \pm 110$ & $4415-3875$ \\
\hline-2963 & $\begin{array}{l}\text { Struct } 45 \\
\quad \text { Ritual pit }\end{array}$ & Cerny & 0.3 & $5420 \pm 180$ & $4545-3885$ \\
\hline-2719 & Struct 49 & $\begin{array}{l}\text { Danubian or } \\
\text { Cerny }\end{array}$ & 0.3 & $5710 \pm 180$ & $5050-4125$ \\
\hline-2717 & $\begin{array}{l}\text { Struct } 3 \\
\text { Advanced } \\
\text { ditch }\end{array}$ & $\begin{array}{l}\text { Danubian Late } \\
\text { Rubané }\end{array}$ & 0.2 & $5920 \pm 260$ & $5295-4320$ \\
\hline-2716 & $\begin{array}{l}\text { Struct } 2 \text { Main } \\
\text { ditch }\end{array}$ & $\begin{array}{c}\text { Late Rubane of } \\
\text { Paris basin }\end{array}$ & 1 & $6080 \pm 110$ & $5270-4740$ \\
\hline-2720 & $\begin{array}{l}\text { Struct } 4 \\
\quad \text { Refuse pit }\end{array}$ & Late Rubane & 1 & $5950 \pm 120$ & $5210-4565$ \\
\hline
\end{tabular}


(Hutrelle \& Piningre, 1978), or Pincevent at La Grande Paroisse, Gif-5005: $5630 \pm 120$ (unpub), or La Grève de Frécul at Barbuisse-Courtavent, Ly2455: 5530 $\pm 150(\mathrm{R}, 1983, \mathrm{v} 25, \mathrm{p} 108)$. Third phase of occupation is Chassean Middle Neolithic. Three dates from this period, Ly-2718, -2714, -2715 , are similar to those from Chassean levels in Boury-en-Vexin and Catenoy sites (above). Fourth phase of site occupation, corresponding to Late Neolithic, has previously been dated by Louvain, Lv-1221:4250 \pm 75 (unpub). Ly-2962 corresponds to Chalcolithic period, probably of "Champs d'Unnes" type. Thus, all these results summarize well entire Neolithic chronology for Paris basin (Blanchet, 1983).

$6590 \pm 140$

Ly-2677. La Madeleine, Pont du Château, Puy de Dôme *5835-5220BC

Human bones from burial in isolated ditch in silty colluvia overlying low terrace of Allier R $\left(45^{\circ} 49^{\prime} \mathrm{N}, 3^{\circ} 15^{\prime} \mathrm{E}\right)$. Coll 1981 by Lab Regional Ponts et Chaussćes, Clermont-Ferrand and subm 1981 by JP Daugas, Dir Antiquités Prehist, Clermont-Ferrand. No assoc industry $(0.7 \mathrm{dr})$. Comment ( JPD): date confirms Neolithic suggested by burial loc and osteol study by A Gilbert (Daugas, Gilbert, \& Raynal, 1982). Date is comparable to Le Creux Rouge sepulture at Clermont-Ferrand, Ly-1944: 6070 \pm 140 (R, 1983, v 25, p 108).

\section{Abri de Strette series, Babaghju, Haute Corse}

Charcoal from two levels in rock shelter $\left(42^{\circ} 42^{\prime} \mathrm{N}, 9^{\circ} 12^{\prime} \mathrm{E}\right)$. Coll 1982 and subm 1983 by J Magdeleine, Bastia.

\section{Ly-2835. Strette No. 4}

$6420 \pm 300$

From Layer XXb, assoc with Cardial Early Neolithic industry $(0.1 \mathrm{dr})$.

\section{Ly.2836. Strette No. 5}

$6480 \pm 430$

Same layer and assoc industry as previous one $(0.2 \mathrm{dr})$.

\section{Ly-2837. Strette No. 7}

$9140 \pm 300$

From Layer XXIV, assoc with Pre-Neolithe lithic industry $(0.2 \mathrm{dr})$.

General Comment ( jM): average of Ly-2835/2836: $6460 \pm 250$, corresponding to most dates for Cardial Neolithic in Provence, eg, at Château. neuf-I ès-Martigues (below). Ly-2837 confirms Pre-Neolithic origin of industry.

\section{La Font des Pigeons series, Châteauneuf-Lès-Martigues, Bouches- du-Rhônt}

Charcoal from large rock shelter $\left(43^{\circ} 23^{\prime} \mathrm{N}, 5^{\prime} 10^{\prime} \mathrm{E}\right)$. Coll 1979 and subm 1982 and 1983 by J Courtin, Dir Antiquités Prehist, Aix-en-Provence (0.07 dr only for Ly-2823).

Ceneral Comment (JC): three series were previously dated from 1960 excavation by $\mathrm{M}$ Escalon de Fonton (1967). One was done by Köln lab from 
TABLE 33

Chãteauneuf-Lès-Martigues

\begin{tabular}{cclcc}
\hline $\begin{array}{c}\text { Sample } \\
\text { no. }\end{array}$ & Layer & \multicolumn{1}{c}{ Assoc industry } & $\begin{array}{c}\text { Corrected } \\
\text { date } \\
\text { interval } \\
\text { (BC) }\end{array}$ \\
\hline $\begin{array}{c}\text { Ly-2823 } \\
-2824\end{array}$ & 1 & Chassean, Middle Neolithic & $5160 \pm 360$ & $4655-3255$ \\
-2825 & 6 & Chassean, Middle Neolithic & $5460 \pm 130$ & $4525-3920$ \\
-2826 & 12 & Epicardial, Early Neolithic & $5590 \pm 120$ & $4700-4170$ \\
-2827 & 13 & Late Cardial, Early Neolithic & $5900 \pm 140$ & $5205-4465$ \\
-2828 & $16 \mathrm{~A}$ & Middle Cardial, Early Neolithic & $6200 \pm 160$ & $5435-4865$ \\
-2829 & 17 & Early Cardial, Early Neolithic & $6550 \pm 100$ & $5755-5225$ \\
-2830 & $18 \mathrm{G}$ & Early Cardial, Early Neolithic & $6200 \pm 100$ & $5360-4925$ \\
-2831 & 19 & Late Castelnovian, Mesolithic & $7260 \pm 120$ & \\
-2832 & 19 & Late Castelnovian, Mesolithic & $6720 \pm 140$ & $5970-5290$ \\
-2833 & 20 & Late Castelnovian, Mesolithic & $7290 \pm 130$ & \\
\hline
\end{tabular}

charcoal: Kn-208: $6700 \pm$ 200, for Late Cardial level, and Kn-182: $7520 \pm$ 240 for Early Cardial level. Latter result suggested very early appearance of Neolithic industries in region (Escalon de Fonton, 1967). Two other series were done by Lyon lab, 7 dates from bones and charcoal from all levels (R, 1977, v 15, p 526-527), and by Monaco lab, MC-2514: $6050 \pm 100$ from Layer 7-8, MC-2515: $6900 \pm 100$ from Layer 17, and MC-2516: $7220 \pm$ 100 from Layer 18. All dates from Lyon and Monaco indicate much younger date, by ca 1000 , for beginning of Neolithic. New excavation was later carried out by J Courtin to check previous data. New series agrees with Ly and MC results, and with archaeol and chronol data from other sites in region (Courtin, Evin, \& Thommeret, in press).

\section{Mesolithic Period}

\section{France}

\section{La Doue series, Saint-Cernin de Larche, Corrèze}

Charcoal from carbonaceous masses (hearth or discharge zones) from rock shelter $\left(45^{\circ} 5^{\prime} \mathrm{N}, 1^{\circ} 24^{\prime} \mathrm{E}\right)$ (Mazière, 1983). Coll and subm 1980 (Ly2223, -2234) and 1982 by G Mazière, Dir Antiquités Prehist, Limoges.

TABLE 34

La Doue

\begin{tabular}{|c|c|c|c|c|c|}
\hline $\begin{array}{c}\text { Sample } \\
\text { no. }\end{array}$ & Loc & $\begin{array}{l}\text { Depth } \\
(\mathrm{cm})\end{array}$ & DR & $\begin{array}{c}\text { Assoc } \\
\text { industry }\end{array}$ & $\begin{array}{l}\text { Age (BP) and } \\
\text { corrected date } \\
\text { interval }\end{array}$ \\
\hline Ly-2649 & $\mathrm{O} / \mathrm{P}-\mathrm{VI}$ & $214-220$ & 0.25 & Medieval & $\left\{\begin{array}{l}1430 \pm 200 \\
\mathrm{AD} 230-900\end{array}\right.$ \\
\hline-2818 & F-V & $98-100$ & 0.2 & Early Neolithic & $\left\{\begin{array}{l}6390 \pm 290 \\
5790-4745 \mathrm{BC}\end{array}\right.$ \\
\hline-2233 & $\mathrm{H}-\mathrm{V}$ & 160 & 1 & Sauveterrian & $8750 \pm 150$ \\
\hline-2234 & $\mathrm{H}-\mathrm{V}$ & 180 & 1 & Sauveterrian & $8880 \pm 160$ \\
\hline-2819 & $\mathrm{I}-\mathrm{V}$ & $178-182$ & 0.3 & Sauveterrian & $9260 \pm 200$ \\
\hline-2820 & $\mathrm{I}-\mathrm{V}$ & $183-196$ & 0.3 & Sauveterrian & $8980 \pm 210$ \\
\hline-2821 & $\mathrm{I}-\mathrm{V}$ & 203-208 & 0.5 & Sauveterrian & $8860 \pm 210$ \\
\hline-2822 & M-V & $478-480$ & 1 & Late Magdalenian & $11,520 \pm 170$ \\
\hline
\end{tabular}


General Comment (GM): all dates agree with chronol expected from assoc industries. Ly-2649 corresponds to beginning of Middle Ages. Ly-2818 may be correlated with Ly-1600: $7010 \pm 430(\mathrm{R}, 1979, \mathrm{v} 21, \mathrm{p} 440)$ from Layer 3 in nearby Chez-Jugie site at Cosnac, which contains some industry, with "Le Martinet" trapezoid type. Sauveterrian dates are very close, at ca $9000 \mathrm{BP}$. They are identical to those from Les Fieux site at Miers, Lot, Gif4281: $9060 \pm 190$ (unpub) and show that industry is much older than at Chez-Jugie (3 dates at ca 8000 BP). Ly-2822 is also identical to two dates from Chez-Jugie assoc with Magdalenian industry with Azilian characteristics, Ly-1572: 11,840 \pm 580 and Ly-1601: 11,730 \pm 530 .

\section{La Vieille Eglise series, La Balme de Thuy, Haute-Savóie}

Charcoal from lowest levels of rock shelter $\left(45^{\circ} 55^{\prime} \mathrm{N}, 6^{\circ} 17^{\prime} \mathrm{E}\right)$. Coll and subm by JP Ginestet, Thônes.

\section{Ly-1936. La Vielle-Eglise 6A}

$8170 \pm 160$

From Level 6A; coll 1978 and subm 1979 (0.7 dr). Assoc with Tardenoisian Mesolithic industry.

\section{Ly-2619. La Vielle-Eglise 7A}

$9820 \pm 200$

From Level 7A; coll 1981 and subm 1982. Assoc with Tardenoisian Mesolithic industry.

General Comment (JPG): both dates agree perfectly with expected values. They date intermediate levels, $6 \mathrm{~B}$ and 6C, at ca $9000 \mathrm{BP}$. Upper levels, 5A and $5 \mathrm{~B}$, containing Middle and Early Neolithic ceramic industry were previously dated, Ly-1934: $5930 \pm 210$ and Ly-1935: $6500 \pm 230(\mathrm{R}, 1983$, v 25, p 108-109; Bintz et al, 1981).

\section{Allée Tortue series, Fère-en-Tardenois, Aisne}

Charcoal from 4 hearths found in middle of open-air site at ca 30 to $50 \mathrm{~cm}$ depth in sand $\left(49^{\circ} 13^{\prime} \mathrm{N}, 3^{\circ} 31^{\prime} \mathrm{E}\right)$. Coll and subm by JG Rozoy, Charleville-Mézières. Site contains rich Late Tardenoisian Mesolithic flint industry (Rozoy, 1978).

\section{Ly-2738. Allée Tortue J-50}

$4070 \pm 170$ $* 3010-2305 B C$

From deepest hearth J-50; coll 1981 and subm 1982; 0.5 dr despite large sample size before alkaline pretreatment.

\section{Ly-2739. Allée Tortue F-51 NE}

$3470 \pm 130$

From hearth F-51 NE in upper part of archaeol level. Coll 1979 and subm 1982.

\section{Ly-2740. Allée Tortue C-54 NW}

$3100 \pm 210$

From hearth C-54 NW in upper part of archaeol level. Coll and subm 1982. 
Ly-3149. Allée Tortue I-50 SW

From beneath hearth I-50 SW (0.3 dr).

General Comment (JGR): first three samples date hearth to Chalcolithic and Bronze ages and show that hearths are intrusive in Mesolithic upper layer of site. Such disturbance of archaeol layer was indicated by inside discovery of flint arrow with peduncle and wings. Ly-3149 shows hearth with Later Tardenoisian industry overlying older charcoal, demonstrating human presence at site early in Mesolithic period.

Ly-2814. Campagnol des Neiges cave, Gresse, Isère $\quad \mathbf{9 0 1 0} \pm \mathbf{2 0 0}$

Charcoal (Pinus sp) from base of gallery of cave opening at ca $1950 \mathrm{~m}$ alt at foot of Séguret Mt in Les Hauts Plateaux du Vercors massif ( $44^{\circ} 52^{\prime}$ N, $5^{\circ} 31^{\prime}$ E). Coll 1981 and subm 1982 by P Bintz. Gallery contains several skeletons of various animal spp and some Epipaleolithic flints. Comment (PB): date confirms age of flint which indicates temporary presence of man in this deep cave, despite high alt and difficult access of site.

\section{Magdalenian Period}

France

\section{Ly-2531. La Roche cave, Courchapon, Doubs}

$8450 \pm 150$

Bones and fragments of antlers from fill near entrance of cave $\left(47^{\circ} 14^{\prime} \mathrm{N}, 6^{\circ} 46^{\prime} \mathrm{E}\right)$. Coll 1950 to 1955 by P Ripotot and R Seibel (1958), preserved in Dôle Mus and subm by M Campy, Dir Antiquité Prehist, Besançon. Fauna underlay level containing Late Magdalenian industry. Comment (MC): date indicates fauna is not contemporaneous with industry, despite proximity.

Ly-2781. Loubressac cave, Mazerolles, Vienne

$9690 \pm 160$

Bones from only archaeol layer in fill of terrace of small cave ( $46^{\circ} 25^{\prime} \mathrm{N}, 0^{\circ} 41^{\prime} \mathrm{E}$ ). Coll 1947 and subm 1981 by L Pradel, Châtellerault. Assoc with Magdalenian $\mathrm{VI}_{2}$ industry. Comment (LP): date is obviously too young for assoc industry as it corresponds to beginning of Holocene. This discrepancy cannot be explained (Pradel, 1983).

Ly-2314. Le Pré des Forges, Marsangy, Yonne

$8440 \pm 190$

Gastropod shells from lowest and upper parts of Layer 5 in open-air site $\left(48^{\circ} 7^{\prime} \mathrm{N}, 3^{\circ} 18^{\prime} \mathrm{E}\right)$. Coll 1979 and subm 1980 by B Schmider, Lab Prehist, Coll France, Paris. Layer 5 contains faunal bones without collagen and Magdalenian industry. Gif TL date from burned sandstones is ca 11,700 BP. Comment (BS): date is too young but shells were coll from entire layer whereas industry was found in only lowest part of level. It confirms unreliability of terrestrial shells for dating archaeol events (Evin et al, 1980; Schmider, 1979).

\section{Abri de Campalou series, Saint-Nazaire-en-Royan, Drôme}

Bones from rock shelter $\left(45^{\circ} 15^{\prime} \mathrm{N}, 5^{\circ} 12^{\prime} \mathrm{E}\right)$. Coll and subm 1972 by JE Brochier and JL Brochier (1973), Romans/Isère. 
From Layer $2 \mathrm{a}$ in Zone 6-7 (0.07 dr); assoc with Late Magdalenian industry with Azilian characteristics, previously dated, Ly-436: 12,800 \pm $300(\mathrm{R}, 1973$, v 15, p 148). Date was considered too old by ca $1000 \mathrm{yr}$, as typol, sedimentol, and palynol data suggest beginning of Alleröd.

Ly-2285. Campalou 3a

$9400 \pm 500$

From level underlying previous one $(0.2 \mathrm{dr})$; assoc with Late Magdalenian industry attributed to Middle Dryas, ca 12,000 BP.

Ly-1958. Campalou base

$13,400 \pm 350$

From lowest level of fill $(0.7 \mathrm{dr})$; assoc with Magdalenian industry attributed to Early Dryas or Bölling.

General Comment (JEB \& JLB): L.y-2286 and -2285 appear too young by ca $1000 \mathrm{yr}$ while previously pub sample was too old also for unknown reasons. Ly-1958 agrees with expected age.

\section{Ly-2693. Gare de Conduché cave, Bouziès, Lot}

$12,040 \pm 160$

Boncs from only black layer of fill in small cave $\left(44^{\circ} 29^{\prime} \mathrm{N}, 1^{\circ} 39^{\prime} \mathrm{E}\right)$. Coll and subm 1982 by M Lorblanchet, Cabrerets Mus. Assoc with Late Magdalcnian industry with microlithic remains. Comment (ML): date agrees with many others for Late Magdalenian of SW France, except those from nearby site, Sainte-Eulalic at Espagnac, Gif-2193: 10,400 \pm 300 and Gif1697: 10,830 \pm 200 , which suggested prolonged presence of Magdalenian industry in Quercy region. Date does not confirm latter hypothesis and indicates that more measurements on samples from Sainte-Eulalie site are needed.

\section{Caves and rock shelters of Bordelais series, Gironde}

Bones from Magdalenian sites in Bordeaux region (Lenoir 1983). Subm by M Lenoir, Lab Quaternary Geol, Univ Bordeaux I (Table 35a,b).

General Comment (ML): dates are generally as expected from assoc industries. Ly-3148 shows bone-bearing breccia is not part of same archacol assemblage as underlying Middle Magdalenian levels but is contemporaneous with fills of many ncighboring sites containing Late Magdalenian industries, such as Ia Crotte des Fées (undated). Ly-2632 confirms that lowest level is from beginning of Upper Paleolithic; Ly-2699 agrees with previous dates of samples from neighboring rock shelter Arbi l du Moulin Neuf, Level 2a, Ly-2352: 13,570 \pm 260 and Level 2b, Ly-2275: 14,280 \pm $440(\mathrm{R}, 1983, \mathrm{v} 25, \mathrm{p} 114)$. Ly-2701 suggests that bone may come from underlying level.

\section{Roc La Tour 1 series, Monthermé, Ardennes}

Charcoal underlying archaeol level of open-air site $\left(49^{\circ} 54^{\prime} \mathrm{N}, 3^{\circ} 27^{\prime}\right.$ E). Coll and subm 1982 by JG Rozoy. Site contains Late Magdalenian V industry. 
TABLE 35a

Bordelais

\begin{tabular}{|c|c|c|c|c|c|}
\hline Site & Village & Geog coordinates & $\underset{\mathrm{yr}}{\text { Colln }}$ & Ref & Subm yr \\
\hline Abri Faustin & Cessac & $\left(44^{\circ} 44^{\prime} \mathrm{N}, 0^{\circ} 10^{\prime} \mathrm{W}\right)$ & 1975 & M Lenoir & 1982 \\
\hline Vidon & Juillac & $\left(44^{\circ} 49^{\prime} \mathrm{N}, 0^{\circ} 16^{\prime} \mathrm{W}\right)$ & 1950 & E Prot & 1982 \\
\hline Roc de Marcamps & $\begin{array}{l}\text { Prignac and } \\
\text { Marcamps }\end{array}$ & $\left(45^{\circ} 02^{\prime} \mathrm{N}, 0^{\circ} 30^{\prime} \mathrm{E}\right)$ & 1979 & M Lenoir & 1980,1981 \\
\hline $\begin{array}{l}\text { Abri } 2 \text { du Moulin } \\
\text { Neuf }\end{array}$ & $\begin{array}{l}\text { Saint-Quentin } \\
\text { de Baron }\end{array}$ & $\left(44^{\circ} 49^{\prime} \mathrm{N}, 0^{\circ} 16^{\prime} \mathrm{W}\right)$ & 1980 & M Lenoir & 1982 \\
\hline
\end{tabular}

TABLE 35b

Bordelais

\begin{tabular}{|c|c|c|c|c|c|c|}
\hline $\begin{array}{c}\text { Sample } \\
\text { no. }\end{array}$ & Site & Layer & $\mathrm{Sq}$ & Assoc industry & DR & Age (BP) \\
\hline Ly-2700 & Abri Faustin & 1 & $\mathrm{~K}-24$ & Late Magdalenian & 0.7 & $12,370 \pm 220$ \\
\hline-2699 & Abri 2 du Moulin Neuf & 2 & M-13 & Middle Magdalenian & 0.5 & $13,380 \pm 250$ \\
\hline-2701 & Vidon & B & & Late Magdalenian & 0.7 & $14,000 \pm 350$ \\
\hline-3148 & Roc de Marcamps & & Breccia & & 1 & $11,910 \pm 230$ \\
\hline-2290 & Roc de Marcamps & $1 b$ & $\mathrm{~N}-28$ & Late Magdalenian & 1 & $14,200 \pm 190$ \\
\hline-2680 & Roc de Marcamps & 2 & $\mathrm{~N}-29$ & Middle Magdalenian & 0.7 & $13,570 \pm 420$ \\
\hline-2291 & Roc de Marcamps & 2 & $\mathrm{~N}-26-28$ & Middle Magdalenian & 0.7 & $14,910 \pm 240$ \\
\hline-2681 & Roc de Marcamps & $3 \mathrm{~b}$ & Core $\mathrm{S}$ & Middle Magdalenian & 0.3 & $15,700 \pm 450$ \\
\hline-2292 & Roc de Marcamps & $4 c$ & M-30 & Middle Magdalenian & 1 & $17,410 \pm 310$ \\
\hline-2682 & Roc de Marcamps & 8 & $\mathrm{M}-30 \mathrm{~N}-30$ & Aurignacian & 0.5 & $26,520 \pm 830$ \\
\hline
\end{tabular}

\section{Ly-2924. Roc de La Tour N-51}

From Sq N-51.

\section{Ly-2925. Roc de La Tour F-61}

From Sq F-61.

\section{Ly-2926. Roc de La Tour}

From Sq L-50 (0.3 dr).

General Comment (JGR): dates show large-scale disturbance of site, frequent in relatively superficial sites where downward migrations of charcoal can occur via root holes or animal burrows.

\section{Ly-3000. La Garenne, Saint-Marcel, Indre}

$14,270 \pm 270$

Bone of microfaunal spp, mainly Microtus sp, from Sec B1 and B2 in cave $\left(46^{\circ} 36^{\prime} \mathrm{N}, 1^{\circ} 30^{\prime} \mathrm{E}\right)$. Coll 1955 by J Allain, Dir Antiquités Prehist, Bourges, and subm 1983 by R Desbrosse, Centre Natl Recherche Sci, Blanzy and JF Kosłowsky, Archaeol Inst Jagielonski, Krakow, Poland $(0.4 \mathrm{dr})$. Site contains Magdalenain industry with "navettes" found in several other sites in distant points of Europe. Many dates from this site were erratic (R, 1978, v 20, p 49-50). Comment (JK): date confirms feasibility of using microfaunal bones as dating material as collagen content is often higher than in macrofaunal bones. Date falls within range of other sites of 
Arlay, Jura, France (R, 1979, v 21, p 446) and Maszyska cave, near Krakow, Poland (R, 1983, v 25, p 115). It confirms contemporaneity of "à Navette" Magdalenian industry in all three sites despite their distance (Desbrosse $e t$ $a l$, in press). Pollen analysis indicates beginning of warm oscillation attributed to Bölling but date is more likely to be Early Dryas.

\section{Monthaud series, Chalais, Indre}

Bones of several spp from three levels of fill of rock shelter $\left(46^{\circ} 32^{\prime} \mathrm{N}\right.$, $1^{\circ} 2^{\prime}$ E). Coll 1966 and subm 1980 by L Pradel, Châtellerault (Pradel \& Pradel, 1967).

\section{Ly-2757. Monthaud III}

$13,420 \pm 200$

From Layer III, assoc with Late Solutrean industry like one from La Tannerie site, previously dated, Ly-2228: 18,020 \pm 270 (R, 1983, v 25, p 116).

\section{Ly-2758. Monthaud II}

$15,450 \pm 290$

From Layer II assoc with Middle Solutrean industry ( $0.7 \mathrm{dr})$.

\section{Ly-2759. Monthaud I}

$16,970 \pm 300$

From Layer I assoc with Early Solutrean industry like one from Layer $\mathrm{H}$ of Laugerie Haute site, previously dated, GrN-1888: 20,890 \pm 300 (R, 1963, v 5, p 167) (0.5 dr).

General Comment (LP): dates fit strat sequence but are in Early Magdalenian rather than Solutrean time range. Date remains unexplained as these two industries have never been contemporaneous.

\section{Ly-2737. Les Guinards, Creuzier le Vieux, Allier}

$17,420 \pm 330$

Reindeer antler from solifluction flow excavated during work on road $\left(46^{\circ} 11^{\prime} \mathrm{N}, 3^{\circ} 25^{\prime} \mathrm{E}\right)$. Coll 1981 by L Magoda, JP Daugas and JP Raynal and subm 1982 by JP Daugas and JP Raynal. Comment (JPD \& JPR): date suggests Early Magdalenian. Sparse assoc industry with many backed bladelets neither exclude nor confirm such attribution (Daugas \& Raynal, 1982) as deposits such as solifluction flows often involve mixing of materials. Date may be compared with several other results from Velay region, such as Les Cottiers site at Retournac, Ly-719: 18,550 \pm 150 (R, 1975, v 17, p 27) and Le Rond du Barry site at Polignac, Gif-3038: 17,100 4450 (unpub).

\section{Czechoslovakia}

\section{Ly-2553. Pekarna cave, Mokra, Moravia}

$12,940 \pm 250$

Bones (Equus sp) first attributed to Layer $\mathrm{g} / \mathrm{h}$ but probably from only Layer g $\left(49^{\circ} 15^{\prime} \mathrm{N}, 16^{\circ} 41^{\prime} \mathrm{E}\right)$. Coll during excavation 1925 to 1930 (Absolon \& Czierek, 1932), preserved in Moravic Mus, Brno, and subm 1981 by K Valoch, Anthropol Inst, Brno. Assoc with Moravian Magdalenian with "navettes" industry, attributed to Middle Dryas. Comment (KV): date as expected from paleontol study of horse bones by R Musil. 


\section{Spain}

\section{La Riera series, Posada de Lianes, Asturias}

Bones from cave deposit $\left(43^{\circ} 26^{\prime} \mathrm{N}, 4^{\circ} 52^{\prime} \mathrm{W}\right)$. Coll 1977 and subm by LG Straus, Univ New Mexico, and GA Clark, Arizona State Univ.

Ly-1646. La Riera 23

$10,340 \pm 560$

From Level 23, Sq H-10, subm 1978; assoc with Upper Magdalenian industry $(0.3 \mathrm{dr})$.

Ly-1645. La Riera 20

$12,360 \pm 670$ $(0.1 \mathrm{dr})$.

From Level 20, Sq E-9, subm 1978; assoc with Magdalenian industry

\section{Ly-1783. La Riera 1}

$20,360 \pm 450$

From Level 1, Sq G-10, subm 1979; assoc with Pre-Solutrean industry, may be Late Aurignacian.

General Comment (LGS): La Riera sequence was dated with 28 samples by Gakushuin, Cambridge, British Mus, Riverside, and Lyon labs on both bones and charcoal. Ly-1646 agrees with GaK-6982: 10,890 \pm 430, for immediately overlying Level 24 . Ly-1645 provides reasonable estimate for Level 20, although 2 other dates, UCR-1273D, which is far too young and GaK-6980: 17,160 \pm 440 which is conceivable at $2 \sigma$ due to depositional hiatus, both sedimentol and palynol detected at this depth. Ly-1783 falls within statistical error of two other dates for Level 1, UCR-1270A: $19,620 \pm 390$ and $\mathrm{BM}-1739: 20,860 \pm 410$. No Solutrean points were found in this basal layer, which is overlain by 16 Solutrean levels with $11{ }^{14} \mathrm{C}$ dates ranging from $20,970 \pm 620$ to $16,900 \pm 200$ (Straus et al, 1981).

\section{Beginning of Late Paleolithic and Middle Paleolithic Periods}

\section{France}

\section{La Vigne Brun series, Villerest, Loire}

Powder of burned bones (Ly-2151 and -2153) and burned bones from hearths in several areas of open-air habitation site $\left(45^{\circ} 59^{\prime} \mathrm{N}, 3^{\circ} 19^{\prime} \mathrm{E}\right)$. Coll and subm by J Combier and JL Porte, Dir Antiquités Prehist, Lyon. In all secs dated, hearths contain rich Upper Perigordian industry and site generally resembles settlements with wall of mammoth bones discovered in $\mathrm{W}$ Russia (Combier et al, 1982).

General Comment (JC): Ly-2512 comes from much eroded area and is unexplained as it seems there was no human occupation of site after Upper Perigordian period. Ly-2151 and -2153 are too young because powdery material did not allow for thorough cleaning. Sample contained carefully selected large fragments of completely charred bones (Evin, 1982). Thus, last 4 dates mutually agree and suggest relatively short occupation of site at 
TABLE 36

Vigne Brun

\begin{tabular}{rlcccc}
\hline $\begin{array}{c}\text { Sample } \\
\text { no. }\end{array}$ & \multicolumn{1}{c}{ Loc } & $\begin{array}{c}\text { Colln } \\
\text { yr }\end{array}$ & $\begin{array}{c}\text { Subm } \\
\text { yr }\end{array}$ & DR & Age (BP) \\
\hline Ly-2152 & Sec XX & 1979 & 1979 & 0.4 & $16,180 \pm 260$ \\
-2151 & Sq P-16 & 1979 & 1979 & 0.3 & $19,500 \pm 480$ \\
-2153 & Sq 0-16 & 1979 & 1979 & 0.5 & $20,840 \pm 390$ \\
-2637 & Sec VIII-IX & 1981 & 1982 & 0.3 & $23,450 \pm 690$ \\
-2638 & Sec VIII-IX & 1981 & 1982 & 1 & $21,580 \pm 600$ \\
-2639 & Sec VII & 1981 & 1982 & 0.4 & $23,230 \pm 750$ \\
-2640 & Sec I-IX & 1981 & 1982 & 0.1 & $23,500 \pm 1000$ \\
\hline
\end{tabular}

ca 23,000 BP. They also agree with previously pub dates from same site, Ly-391: 24,900 $\pm 2000(\mathrm{R}, 1975, \mathrm{v} 17, \mathrm{p} 29)$ and with other results from two sites in same region that contain similar industry: Saint-Martin-sousMontaigu, Ly-31 1: 22,900 \pm 600 and Ly-309: 24,150 700 (R, 1971, v 13, p 64) and Solutré: 4 dates between 21,600 \pm 700 and 24,050 $\pm 600(\mathrm{R}$, 1971 , v 13, p 63; R, 1973, v 15, p 518).

\section{Le Flageolet I series, Bézenac, Dordogne}

Bones from several archaeol levels of rock shelter $\left(44^{\circ} 51^{\prime} \mathrm{N}, 1^{\circ} 5^{\prime} \mathrm{E}\right)$. Coll 1976 and 1980 by JP Rigaud, Dir Antiquités Prehist, Bordeaux. Site was successively occupied from beginning of Upper Paleolithic until Late Perigordian (Rigaud, 1982).

General Comment ( JPR): use of ammonia contaminated by atmospheric $\mathrm{CO}_{2}$ during lab treatment surely caused all first-measured samples (Ly-1606 to $-1608,-1748,-1749)$ to appear too young. Exact amount of contamination could not be calculated but dates are probably off in proportion to dilution ratio; thus, they must be all considered min ages. All other dates are in mutual agreement and are within expected age range, although some are a little younger than Groningen samples from levels containing similar industries in Abri Pataud rock shelter (R, 1967, v 9, p 114-115; R, 1972, v 14, p 56).

TABLE 37

Le Flageolet I

\begin{tabular}{rrlcrr}
\hline $\begin{array}{c}\text { Sample } \\
\text { no. }\end{array}$ & Layer & Assoc industry & Subm yr & DR & \multicolumn{1}{c}{ Age (BP) } \\
\hline Ly-1606 & I/III & Late Perigordian & 1977 & 0.7 & $\geq 22,440 \pm 680$ \\
-2185 & I/III & Late Perigordian & 1979 & 0.3 & $18,610 \pm 440$ \\
-1607 & IV & Late Perigordian & 1979 & 0.5 & $\geq 21,190 \pm 920$ \\
-2186 & IV & Late Perigordian & 1979 & 0.8 & $22,950 \pm 500$ \\
-2721 & V & Late Perigordian & $1977-1981$ & 0.5 & $22,520 \pm 500$ \\
-2722 & VI & Late Perigordian & $1977-1981$ & 0.6 & $24,280 \pm 500$ \\
-1748 & VII & Late Perigordian & 1977 & 1 & $\geq 25,720 \pm 610$ \\
-2723 & VII & Late Perigordian & $1977-1981$ & 0.3 & $26,150 \pm 600$ \\
-1608 & VIII & Aurignacian & 1977 & 1 & $\geq 23,280 \pm 670$ \\
-2724 & VIII/1 & Aurignacian & 1981 & 0.3 & $26,800 \pm 1000$ \\
-2725 & VIII/2 & Aurignacian & 1981 & 0.8 & $27,350 \pm 1400$ \\
-1749 & IX & Aurignacian & 1977 & 0.2 & $\geq 20,270 \pm 1760$ \\
-2726 & IX & Aurignacian & 1981 & 0.4 & $27,000 \pm 1000$ \\
-2727 & XI & Early Aurignacian & $1977-1981$ & 0.3 & $\geq 31,500$ \\
\hline
\end{tabular}




\section{Le Fonténioux cave series, Saint-Pierre de Maillé, Vienne}

Bones and teeth of animal spp from cave fill $\left(46^{\circ} 40^{\prime} \mathrm{N}, 0^{\circ} 50^{\prime} \mathrm{E}\right)$. Coll 1951 and subm 1982 by L Pradel.

Ly-2785. Fonténioux, No. 2

From level containing Upper Aurignacian V industry.

Ly-2784. Fonténioux, No. 1

$25,400 \pm 450$

From level containing Upper Perigordian IVa industry $(0.7 \mathrm{dr})$.

General Comment (LP): both dates agree perfectly with expected age (Pradel, 1953). They confirm generally assumed contemporaneity of Late Aurignacian and Perigordian. They also agree with dates from neighboring Laraux site, Ly-1740: 23,510 $\pm 640(\mathrm{R}, 1979, \mathrm{v} 21, \mathrm{p} 447)$.

\section{Le Raysse series, Brive-La-Gaillarde, Corrèze}

Bones of several animal spp from rock shelter fill $\left(45^{\circ} 7^{\prime} \mathrm{N}, 1^{\circ} 32^{\prime} \mathrm{E}\right)$. Coll 1964 and subm 1982 by L Pradel (Pradel \& Pradel, 1966).

Ly-2782. Le Raysse, No. 4

$25,000 \pm 660$

From Layer 4 ; assoc with Upper Perigordian $\mathrm{V}_{\mathrm{C}}$ industry $(0.5 \mathrm{dr})$.

\section{Ly-2783. Le Raysse, No. 3}

$23,630 \pm 480$

From Layer 3; assoc with Aurignacien I industry ( $0.5 \mathrm{dr})$.

General Comment (LP): Ly-2782 is within age generally accepted for Late Perigordian industry but Ly-2783 is obviously too young for Early Aurignacian.

Ly-2752. Les Cottés, Saint-Pierre de Maillé, Vienne $\quad \mathbf{2 3 , 4 2 0} \pm \mathbf{7 1 0}$

Bones from animal spp from Layer IV of terrace fill of cave $\left(46^{\circ} 40^{\circ} \mathrm{N}\right.$, $0^{\circ} 50^{\prime} \mathrm{E}$ ). Coll 1958 to 1960 and subm 1980 by L Pradel. Assoc with evolved Early Perigordian industry (Pradel, 1961). Comment (LP): date is much younger than those from Layers I, G, and E, dated by Groningen (R, 1967, $\mathrm{v} 9, \mathrm{p} 107-155)$. It is too young to be attributed to Early Perigordian, but sterile layer overlying Layer E in cave contains scattered Perigordian IVa industry to which date could correspond.

\section{Dousse series, Angles-sur-l'Anglin, Vienne}

Bones from fill of two rock shelters in close proximity $\left(46^{\circ} 42^{\prime} \mathrm{N}, 0^{\circ} 51^{\prime}\right.$ E). Coll 1957 and subm 1980 by L Pradel.

\section{Ly-2753. Abri Sabourin}

$29,300 \pm 800$

From only archaeol level of rock shelter; assoc with Mousterian industry without bifaces.

Ly-2755. Abri Rousseau II-3

From Level II, Layer 3, upper layer of fill of rock shelter; assoc with Mousterian industry without handaxes, more evolved than previous one. 
Ly-2754. Abri Rousseau, I-2

From Level I, Layer 2, lower layer of fill of rock shelter; assoc with Mousterian industry without handaxes.

General Comment (LP): Ly-2753 is rather young for Middle Paleolithic industry or could indicate localized continuation of Mousterian contemporaneity with Early Perigordian and Aurignacian industries in neighboring sites, $i e$, at Les Cottés (above). Ly-2755 and -2754 confirm that this Mousterian industry is older than Würmian III stadial.

\section{Ly-2756. L'Ermitage, Lussac-les-Châteaux, Vienne}

$26,600 \pm 600$

Bones of animal spp from only level of cave fill $\left(46^{\circ} 23^{\prime} \mathrm{N}, 0^{\circ} 43^{\prime} \mathrm{E}\right)$. Coll 1953 and subm 1980 by L Pradel. Assoc with Mousterian industry of La Quina or Charentian type (Pradel \& Pradel, 1954) $(0.8 \mathrm{dr})$. Comment (LP): for unknown reason, date is too young even for very Late Middle Paleolithic.

\section{Ly-2902. Abri Moula, Soyons, Ardèche}

$20,060 \pm 320$

Bones from several sqs at $5.4 \mathrm{~m}$ depth in fill of rock shelter $\left(44^{\circ} 43^{\prime} \mathrm{N}\right.$, $4^{\circ} 50^{\prime}$ E). Coll 1982 by Archaeol Club, Crouzet Cie, and P Payen, Valence. Assoc with Mousterian industry. Comment (PP): date is younger than previous result, Ly-2488: 32,200 \pm 1500 from overlying level which also gave similar date, Ly-2217: 20,100 $\pm 310(\mathrm{R}, 1983, \mathrm{v} 25, \mathrm{p} 118)$.

\section{Les Pècheurs series, Casteljau, Ardèche}

Bones from superimposed hearths $(\mathrm{F})$ of habitation level, from base (S) of fill, from layer at presumed base of isolated hut (L) (Lhomme, Enétério, \& Moulin, 1980) in rock shelter $\left(44^{\circ} 24^{\prime} \mathrm{N}, 4^{\circ} 23^{\prime} \mathrm{E}\right)$. Coll 1976 to 1981 and subm 1978 and 1981 (Ly-2343) by G Lhomme, Les Vans.

General Comment (GL): Ly-2339 and -2342 are obviously much too young by ca $10,000 \mathrm{yr}$. Although other values seem to agree with strat sequence confirmed by amino acid racemization of bones (Lafont, 1984), they all seem a little too young for French sites from beginning of Late Paleolithic, either in Perigord (eg, at Les Eyzies) or in Bourgogne (eg, at Arcy-sur-Cure) (Delibrias \& Evin, 1974). This general tendency to younger ages remains unex-

TABLE 38

Les Pècheurs

\begin{tabular}{rclccc}
\hline $\begin{array}{c}\text { Sample } \\
\text { no. }\end{array}$ & Loc & Assoc industry & $\begin{array}{c}\text { Depth } \\
(\mathrm{cm})\end{array}$ & DR & Age (BP) \\
\hline Ly-2337 & F-9 & Aurignacian I & $210-230$ & 0.5 & $26,760 \pm 1000$ \\
-2338 & F-11-12 & $\begin{array}{c}\text { Aurignacian 0 } \\
\text { Early Aurignacian }\end{array}$ & $225-230$ & 0.6 & $29,400 \pm 900$ \\
-2339 & F-10-11 & $\begin{array}{l}230-240 \\
\text { Early Aurignacian } \\
\text { or Mousterian }\end{array}$ & $270-300$ & 0.4 & $23,880 \pm 750$ \\
-2340 & L-1-2 & Early Aurignacian \\
or Mousterian & $240-250$ & 0.5 & $28,400 \pm 900$ \\
-2341 & F-13 & $\begin{array}{c}\text { Early Aurignacian } \\
\text { or Mousterian } \\
\text { Mousterian }\end{array}$ & 260 & 1 & $\geq 31,000$ \\
-2343 & F-16 & $395-420$ & 0.7 & $24,940 \pm 680$ \\
-2342 & 5-base & &
\end{tabular}


plained, maybe due to contaminant in bones which was not eliminated during normal pretreatment.

\section{La Baume de Gigny series, Gigny-sur-Suran, Jura}

Bones from middle fill of rock shelter $\left(46^{\circ} 27^{\prime} \mathrm{N}, 5^{\circ} 27^{\prime} \mathrm{E}\right)$. Coll and subm by $M$ Vuillemey, Lons-le-Saunier, to complete and check earlier series (see, eg, R, 1979, v 21, p 447) and to confirm presence of sedimentation hiatus or erosion level beneath Layer VIII, as suggested by sedimentol study (Vuillemey, in press). Later, Layer VIII yielded Ly-789: 28,500 \pm 1400 and Ly-566: 29,500 \pm 1400 , and contains Late Mousterian industry.

\section{Ly-3063. La Baume de Gigny, IX}

Macrofaunal bones from Layer IX, just overlying presumed hiatus or erosion level $(0.1 \mathrm{dr})$.

Ly-1701. La Baume de Gigny, $X$

$27,000 \pm 1400$

Microfaunal bones from small Layer X, interbedded with previous one on side of fill, against rockshelter wall.

\section{Ly-2526. La Baume de Gigny, XI}

Microfaunal bones from Layer XI, same level as Ly-3063 (0.15 dr).

General Comment (MB): Ly-1701 is too young for its strat position. Sample may include bones from overlying levels. Ly-3063 and -2526 demonstrate great difference in age between Layer VIII and IX as suggested by sedimentol study and organic content of bones, which is 6 times lower in lowest level of fill, below Layer VIII (Evin, in press).

\section{Saint-Marcel cave series, Bidon, Ardèche}

Samples from fill at entrance of rock shelter $\left(44^{\circ} 19^{\prime} \mathrm{N}, 4^{\circ} 37^{\prime} \mathrm{E}\right)$. Coll and subm by R Gilles, Saint-Marcel d'Ardèche. Assoc with Mousterian industry.

\section{Ly-2276. Saint-Marcel E}

$29,330 \pm 650$ dr).

Bones from Layer E at ca 2.3m depth; coll 1976 and subm 1979 (0.7

Ly-2861. Saint-Marcel G1

$23,260 \pm 370$

Bones from Layer $\mathrm{G}$ at ca 2.5m depth; coll 1979 and subm 1982.

Ly-2901. Saint-Marcel G2

Charcoal from Layer G; coll 1979 and subm 1982; 0.1 dr due to extensive dissolution during alkaline pretreatment.

General Comment (RG): Ly-2861 is much too young for assoc industry and cannot be explained. Ly-2901 is too diluted to be conclusive; Ly-2276 is younger than expected but should indicate continuation of Mousterian industry in region. 


\section{Czechoslovakia}

\section{Dolni-Vestonice series, Mikulov, S Moravia}

Charcoal from center of open-air site $\left(48^{\circ} 48^{\prime} \mathrm{N}, 16^{\circ} 39^{\prime} \mathrm{E}\right)$. Coll 1975 and 1977 at base of Würmian III loess at ca $4.5 \mathrm{~m}$ depth and subm by $\mathrm{B}$ Klima, Aücsav, Brno. Assoc with Gravettian industry of Pavlovian type (Klima, 1981).

\section{Ly-1303. Dolni Vestonice, No. 1 \\ $22,250 \pm 570$ \\ Ly-1999. Dolni Vestonice, No. 2 \\ $19,640 \pm 540$}

General Comment (BK): dates are much younger than expected, ca 25,000 $\mathrm{BP}$, corresponding to Tursac interstadial, $i e$, only a little younger than Gro1286: 25,600 \pm 170 (Klima, 1981) from 1928-1929 excavation. According to strat data, such a great discrepancy, ca $3000 \mathrm{yr}$, cannot be explained by contamination or mixing of material.

\section{Poland}

\section{U1 Spadzista series, Krakow}

Burned bones from Layer 6, Loc C2 in open-air site $\left(50^{\circ} 4^{\prime} \mathrm{N}, 19^{\circ} 57^{\prime}\right.$ E). Coll and subm 1980 by JK Kozłowski. From silty solifluction layer at base of last Würmian loess or Upper Late Loess. Assoc with Gravettian industry of Kostenk Avdeyevo type.

\section{Ly-2541. U1 Spadzista No. 1}

$17,400 \pm 310$

From Sqs B1 and B2 at top of Layer 6, underlying base of Layer 5, which is most typical Late Loess, at ca $480 \mathrm{~cm}$ depth under present soil level, and at 222 to $228 \mathrm{~cm}$ from excavation ref level " 0 " ( $0.6 \mathrm{dr})$.

\section{Ly-2542. U1 Spadzista No. 2}

$$
21,000 \pm 900
$$

From Sq B7 at base of Layer 6 at ca $420 \mathrm{~cm}$ under present surface, and at 127 to $137 \mathrm{~cm}$ from excavation ref level " 0 ."

General Comment (JKK): Ly-2542 is close to Ly-631: 20,600 \pm 1050 (R, $1975, v 17, \mathrm{p} 29)$ from Loc B in same site, at base of same Layer 6. Ly-2541 is, as expected, younger than Ly-2542, and marks beginning of typical Late Loess deposits. Apparent inversion of dates with regard to depth occurs because soliflucted sediments slope downward in comparison to present soil level surface.

\section{REFERENCES}

Absolon, K and Czizek, R, 1932, Die paleolithische Erforschung der Pekarna-Höle in Mähren Dritter Bericht für das Jahr 1927: Cas Moravského zemskẻho mus 26-27, p 479-599.

Allain, J, 1981, Information archéologiques, circonscription du Centre: Gallia Préhist, v 24, no. 2, p 329-363.

Allibert, C, Argant, A, and Argant J, 1983, Le site de Bagamoyo, Mayotte, Archipel des Comores: Etude Océan Indien, documents comoriens, Inst Hist Civilisation Orientale, p 540. 
Arn, R, 1981, Sur les dépôts glaciaires du Signal de Bougy et de la Côte (VD): Ecol Helvetica, v 74, no. 2, p 561-568.

_ _ in press a, Le Quaternaire de la région de Bière (VD): mise en évidence d'une oscillation rhodanienne finiwürmienne: Lab Univ Lausanne, in press.

- in press b, Les formations quaternaires de la Drance de Savoie, nouvelles données et interprétations: Ecol Géol Helvetica, in press.

Bachelery, P, (ms), 1981, Le Piton de la Fournaise, Ile de la Réunion: Etude volcanologique, structurale et pétrologique: PhD dissert, Univ Clermont-Ferrand, $150 \mathrm{p}$.

Barrière, B, 1983, Aubazine: Monastère de Coyrou: Travaux Archéol Limousine, v 3, p 102.

Beaulieu, JL de, Evin, J, Mandier, P, Monjuvent, G, and Reille, M, 1980, Les Echets: un marais capital pour l'histoire climatique du Quaternaire rhodanien: Mém Mus hist nat Paris, ser B, v 27, p 123-136.

Beaulieu, J L de, Pons, A, and Reille, M, in press, Recherches pollenanalytiques sur l'histoire de la végétation du Cézalier, de la Planèze de Saint-Flour et de la Margeride, Massif Central, France: Pollen et Spores, in press.

- in press, Recherches pollenanalytiques sur l'histoire de la végétation des monts d'Aubrac, Massif Central: Rev Paléobot Palynol, in press.

Beaulieu, JL de and Reille, M, 1984, A long Upper Pleistocène pollen diagram from Les Echets, near Lyon, France: Boreas, v 13, p $111-132$.

Beeching, A and Thomas-Beeching, J, 1982, sondage à Rochas; Fèd Ardèchoise, Recherche Préhist Bull, v 7, p 4-5.

Ben Tiba, B, (ms), 1980, Contribution pollenanalytique à l'histoire Holocène de la végétation de Kroumirie (Tunisie septentrionale): PhD dissert, d'Aix-Marseille III, 76 p.

Ben Tiba, B and Reille, M, 1982, Recherches pollenanalytiques dans les montagnes de Kroumirie (Tunisie septentrionale): premiers résultats: Ecol mediterranea, v 8, no. 4, p 7586.

Berthier, S, (ms), 1983, Etude archéologique d'un secteur d'habitat à Koumbi Saleh, Mauritanie: PhD dissert, Univ Lyon II, 243 p.

Bidet, J C and Carruesco, C, 1980, La lagune de Moulay Bou Salhem (Côte atlantique du Maroc): Introduction à l'étude d'un milieu sédimentaire actuel et sub-actuel: Soc Géol Fr Comptes rendus, v 1980 , no. 2 , p 52-53.

Bintz, P, Ginestet, J P, Mourre, J, and Pion, G, 1981, L'homme et son environnement à l'Epipaléolithique dans les Alpes du Nord, d'après les données récentes: Soc Préhist Fr Bull, v 78, p 130-131.

Bizot, B, Serralongue, J, and Serralongue, $\mathrm{H}$, in press, Albigny Condion, basilique funéraire des VIème et VIIIème S: Atlas Monuments Paléochrétiens France, Ministère Culture, in press.

Blanc, C, 1982, Lons-Tumulus 2-3-4-5-6, informations archéologiques de la circonscription d'Aquitaine: Gallia-Préhist, v 25, no. 2, p 432.

Blanchet, J C, 1983, Un exemple de l'apport des dates ${ }^{14} \mathrm{C}$ corrigées, Néolithique Final et Chalcolithique dans le Bassin Parisien et le Nord de la France: Rev Archéomètrie, v 1983 supp, p 85-91.

Blanchet, J C and Decormeille, A, 1980, Le site du "Coq Galleux" et du "Hazoy" à Compiègne (Oise): Cahiers Archéol Picardie, v 7, p 49-84.

1984, Le Camp Chasséen de Catenoy (Oise), premiers résultats: Rev Archéol Picardie, v 1, p 173-205.

Blanchet, J C, Decormeille, A, and Marquis, P, 1980, Récentes découvertes du Néo-Danubien dans la Moyenne Vallée de l'Oise: Préhist Protohist Champagne-Ardennes, v 4, p 5-21.

Blanchet, J C, Martinez, R, and Meniel, P, 1982, Deux nouveaux sites chasséens dans l'Oise: le site fortifié de Boury-en-Vexin et l'habitat de Pont-Sainte-Maxence: Soc Préhist Fr Bull, v 79, p 7-9.

Bonnamour, L, 1978-1979, Chronique aechéologique: Mém Soc Hist Archéol Chalon-surSaône, v 48, p 29.

1980, Découvertes archéologiques en Saône sur le site d'Ormes-Boyer (S \& L): Mém Soc Hist Archéol Chalon-sur-Saône, v 49, p 51-74.

1981, Chronique archéologique: Mém Hist Archéol Chalon-sur-Saône, v 50, p $16-17$. v $1, \mathrm{p} 38-43$.

Bravard, J P, 1983, Une autocapture du Rhône par déversement dans les basses terres du Bas-Dauphiné (Isère, Ain): Rev Géog Lyon, v 58, p 369-382.

Brochier, J E and Brochier, J L, 1973, L'art mobilier de deux nouveaux gisements Magdaléniens à Saint-Nazaire en Royans (Drôme): Etudes Préhist, v 4, p 1-12.

Brun, A, 1977, Données floristiques et paléoclimatologiques du Pléistocène supérieur dans le Chablais (Haute-Savoie): Résultats synthétiques et chronostratigraphiques: Assoc Fr Etudes Quaternaire Bull, v 52, p 39-54. 
Bruneaux, J L, Méniel, P, and Rapin, A, 1980, Un sanctuaire gaulois à Gournay sur Aronde (Oise): Gallia, v 38, no. 1, p 1-25.

Burel, T, Klingebiel, A, and Vernette, G, 1982, Indices d'une ligne de rivage émergée dans la région de Catagena (Colombie): Inst Geol Bassin Aquitaine Bull, v 29, p 161-164.

Burollet, P F, Clairefond, P, and Winnock, E, 1979, La Mer Pélagienne: Geol Méditerranéenne, v 6 , no. 1,345 p.

Cantié, $G$, in press, Le site castral du Dognon (Commune de Châtenet-en-Dognon, HauteVienne): Travaux Archéol Limousine, v 5, in press.

Carité, D, 1983, Essai de synthèse du Quaternaire marin récent (40 000 ans à l'actuel) de Tin Ouéich $\left(18^{\circ} 4^{\prime} \mathrm{N}, 15^{\circ} 49^{\prime} \mathrm{W}\right.$, Mauritanie): Geobios, v 16, p 381-386.

Chertier, B, 1981, Informations archéologiques de la circonscription Champagne-Ardennes: Assenay: Gallia Préhist, v 1981, no. 2, p 444-446.

Clot, A, 1984, La faune de la grotte préhistorique du Bois du Cantet, Espèche, HautesPyrénées, France: Munibe, v 36, p 33-50.

Clot, A and Evin, J, 1983, Premiers datages ${ }^{14} \mathrm{C}$ faunes naturelles du Pléistocène supérieur et de l'Holocène des Pyrénées Occidentales: Soc Préhist Fr Bull, v 80, no. 6, p 164-165.

Colardelle, R and Colardelle, M, 1980, L'habitat médiéval immergé de Colletières à Charavine (Isère): Rev Archéol Médiéval, v 10, p 167-269.

Combier, J, Hayrols, P, Porte, J L, and Gely, B, 1982, Etat actuel des recherches à la Vigne Brun, Villerest, Loire: Les habitats du Paléolithique supérieur: Actes colloque internatl Roanne-Villerest, v 2, p 274-281.

Constantin, C and Lassere, E M, 1983, Chronologie fine du Néolithique et datation radiocarbone: Rev Archéomètrie, v 1983 supp, p 93-99.

Coquerel, R and Pousthamis, B, 1977, Fouilles sur un cimetière à Agos-Vidalos (H P): Soc Ramond Bull, v 1977, p 97-110.

Courtin, J, Evin, J, and Thommeret, U, in press, Révision de la chronologie absolue du site de Chateauneuf-les-Martigues: Gallia Préhist, in press.

Dalongeville, R and Sanlaville, P, 1981, Mission Soudano-française dans la province de la Mer Rouge (Soudan), rapport de mission 1981: Maison Orient, Univ Lyon II, p 21-28.

Daugas, J P, Gilbert A, and Raynal, J P, 1982, Premières sépultures du Néolithique ancien en Basse-Auvergne: Soc Anthropol Sud-Ouest Bull, v 18, p 44-52.

Dagas, J P and Raynal, J P, 1982, Informations archéologiques de la région Auvergne: Gallia Préhist, v 25, p 361 .

Decker, E, Guillaume, C, and Michels, R, 1977: Le gisement Rubané linéaire récent du "Dolmen" (Kirschnaumen-Evendorff, Moselle) datation C 14: Soc Préhist Fr Bull, v 72, p 155-160.

Delibrias, G and Dutil, P, 1966, Formations calcaires lacustres du Quaternaire supérieur dans le Massif Central Saharien (Hoggar) et datations absolues: Acad Sci (Paris) Comptes rendus, ser D, v 262, p 55-58

Delibrias, $G$ and Evin, J, 1974, Sommaire des dates ${ }^{14} \mathrm{C}$ concernant la préhistoire en France: I: dates parues de 1955 à 1974, chapitre 1, Paléolithique Moyen et Paléolithique Supérieur: antérieur à $10000 \mathrm{BP}(8000 \mathrm{BC})$ : Soc Préhist Fr Bull, v 71, p 149-156.

Delibrias, G, Guillier, M T, and Labeyrie, J, 1965, Saclay natural radiocarbon measurements II: Radiocarbon, v 7, p 236-244.

1969, Gif natural radiocarbon measurements III: Radiocarbon, v 11, p 327-344. 1971, Gif natural radiocarbon measurements VI: Radiocarbon, v 13, p 213-254.
1974, Gif natural radiocarbon measurements VIII: Radiocarbon, v 16, p 15-94.

Desbrosse, R, Allain, J, Kosłowski, J K, and Rigaud, J P, in press, Le Magdalénien à Navettes: Gallia Préhist, v 28, in press.

Deyber, A, Dalaut, M, Ladier, E, and Weiseock, A, in press, L'habitat fortifié de "La Pierre d'Appel" à Etival-Clairefontain (Vosges): Gallia, v 42, no. 1, in press.

Diop, A, 1977, Institut Fondamental d'Afrique Noire radiocarbon dates II: Radiocarbon, v 19, p $161-164$.

Doornkamp, J C, Brunsden, D, and Jones, D K C, 1980, Geology, geomorphology and pedology of Bahrain: Norwich editions, $443 \mathrm{p}$.

Dubar, M, (ms), 1983, Stratigraphie des dépôts du Néogène supérieur et du Pléistocène du bassin de la moyene Durance; interprétation géodynamique et paléogéographique: $\mathrm{PhD}$ dissert, Univ Provence, Marseille, 428 p.

Escalon de Fonton, M, 1967, Datation C 14 du Cardial ancien de Chateauneuf: Soc Préhist Fr Bull, v 64, p 101 .

Evin, J, 1982, Datations par le radiocarbone du site de Vigne Brun à Villerest, Loire: Les habitats du Paléolithique supérieur: Actes colloque internat, Roanne-Villerest, v 1, p 148149 .

d'origine 1983, Materials of terrestrial origin used for radiocarbon dating-Les matériaux d'origine terrestre utilisés pour les datations par le radiocarbone, in Internatl ${ }^{14} \mathrm{C}$ and archaeol conf, 1st, Proc: PACT, v 8, p 235-276. 
Evin, J, in press, Les datations radiocarbone du site de la Baume à Gigny-sur-Suran: Cahiers Recherche Solutré, in press.

Evin, J, Longin, R, Marien G, and Pachiaudi, C, 1971, Lyon natural radiocarbon measurements II: Radiocarbon, v 13, p 52-73.

Evin, J, Maréchal, J, and Marien, G, 1983, Lyon natural radiocarbon measurements IX: Radiocarbon, v 25, p 59-128.

Evin, J, Marien, G, and Pachiaudi, C, 1973, Lyon natural radiocarbon measurements III: Radiocarbon, v 15, p 134-155.

- 1973, Lyon natural radiocarbon measurements IV: Radiocarbon, v 15, p $514-$ 533.

1975, Lyon natural radiocarbon measurements V: Radiocarbon, v 17, p 4-34.

88 1976, Lyon natural radiocarbon measurements VI: Radiocarbon, v 18, p 60

88.

1978, Lyon natural radiocarbon measurements VII: Radiocarbon, v 20, p 19-

57.

452.

Evin, J, Maréchal, J, Pachiaudi, C, and Puisségur, J J, 1980, Conditions involved in dating terrestrial shells, in Stuiver, $M$ and Kra, R, Internatl radiocarbon conf, 10 th, Proc: Radiocarbon, v 22, no.2, p 545-555.

Evin, $\mathrm{J}$ and Olive, $\mathrm{P}$, in press, Datation par le ${ }^{14} \mathrm{C}$ du site de Colletière à Charavine (Isère): Dossiers Archéol, in press.

Fagès, $G, 1982$, Le gisement préhistorique et protohistorique de Baume Layrou: Spelunca, v $5, \mathrm{p} 27-28$.

Gaiffe, M, 1983, La fuite des terres du Jura, un problème lié au karst: Pubs Centre Univ études régionales, Univ Franche-Comté, p 1-19.

Galigniér, H and Thureau, C, 1976, Fouilles archéologiques sur le site de St-Pierre-le-Puellier à Tours, 1969-1974 rapport préliminaire: Soc Archeol Touraine Bull, v 38, p 155-172.

Gasco, J and Binder, D, 1983, Série dates radiocarbone et représentation graphique: Rev Archéomètrie, supp, p 75-84.

Gassler, A, 1982, Spätbronzezeitliche keramik vom Wittnauer Horn: Archäol Korrespondenzblatt, v 12, p 55-67.

Graindor, M J, 1966, Terrasse du Haut-Normannien à Bardouville (Seine-Maritime): Science, v 43, p 3-12.

Grappin, S, 1982, Les recherches à Saint-Romain: Mém Comm Antiquités Côtes-d'Or, v 32, p $100-103$.

Guadagnin, R, in press, La Villa de Villarem, études archéologiques et anthropologiques d'une communauté rurale du Parisis aux époques mérovingiennes et carolingiennes, (VIIème IXème): Archéol Médiévale, in press.

Guérin, C, Philippe, M, and Vilain, R, 1979, Le gisement Pléistocène de la grotte de Jaurens à Nespouls, Corrèze, France: historique et généralités Nouvelles Archives Mus Hist Nat Lyon, v 17, p 11-16.

Guillaumet, J P and Maranski, D, 1982, Bilan des fouilles archéologiques en Côte d'Or, la Nécropole de Thury: Mém Comm Antiquité Côte d'Or, v 32, p 30-38.

Houdré, J J and Vital, J, 1981, La fosse Protohistorique de Caramontron: le Basisn du Puy au temps préhistorique: Mus Crozarier du Puy, p 156-162.

Hurtrelle, J and Piningre, J F, 1978, Datation radiocarbone du Cerny des Sablins à Etaples (Pas-de-Calais): Soc Préhist Fr Bull, v 75, p 83-86.

Jeannet, A, 1981, Un habitat du Premier Age du Fer à Jugy (S \& L): Mém Soc Hist Archéol Châlon, v 50, p 27-41.

Joussaume, R, Jauneau, J M, Boiral, M, Robin, P, and Gachina, J, 1979, Néolithique ancien du Centre-Ouest; Note préliminaire: Soc Préhist Fr Bull, v 76, p 178-183.

Klein, J, Lerman, J C, Damon, P E, and Ralph, E K, 1982, Calibration of radiocarbon dates: tables based on the consensus data of the Workshop on Calibrating the Radiocarbon Time Scale: Radiocarbon, v 24, p 103-150.

Klima, B, 1981, Der Mittlere Teil der paläolithischen Station bei Dolni Vestonice: Pamàtky Archeol, v 72, p 5-92.

Labeyrie, J and Delibrias, G, 1976, Niveaux marins récents à vermitidae du littoral OuestAfricain: Assoc Etude Quaternaire Bull, v 47, p 97-109.

Lafont, R, (ms), 1984, Racémisation des acides aminés du collagène d'ossement fossiles: Application aux datations absolues: PhD dissert, Univ Lyon 1, 78 p.

Lechevalier, in press, Etude d'un milieu humide des Collines de Normandie: la tourbière du Pré-Maudit de Gathémo: Soc Linnéenne Normandie Bull, v 111, in press.

Lefrèvre, D, (ms), 1985, Les formations Plio-Pleistocène du bassin de Ksali, moyenne Moulouya, Maroc: PhD dissert, Univ Bordeaux I, $150 \mathrm{p}$.

Lenoir, M, (ms), 1983, Le Paléolithique des Basses-Vallées de la Dordogne et de la Garonne: PhD dissert, Univ Bordeaux I, $702 \mathrm{p}$. 
Lepage, L, 1980, Le Camp de la Vergentières à Cohons (Haute Marne), rapport avec les régions avoisinantes: Préhist Protohist Champagne-Ardennes, v 4, p 139-165.

1982, Le Dolmen II du Camp de la Vergentière à Cohons: Préhist Protohist Champagne-Adrennes, v 6, p 59-69.

Lhomme, G, Enétério, S, and Moulin, D, 1980, Le site de Casteljau, campagne de fouilles 1980: Nouvelles Archives Mus Hist Nat Lyon, v 18, supp, p 35-39.

Lugan, B, 1983, Le Rwanda Oriental: Archéologie du Rwanda: Tervuren, Presse Mus Royal, p $130-136$.

Lugan, B, Sirven, P, and Vérin, P, 1979, Etude de la Grotte dite de Rwabugiri Parc National de l'Akagéra (Rwanda): Azania, v 14, p 148-154.

Mandier, P, 1981, Le Marais des Echets: premiers enseignements pour l'histoire climato-stratigraphique du Quaternaire lyonnais: Lab Rhodanien Geomorphol Bull, v 9, p 39-61.

Mauny, R, 1951, Etat de la question du Ghana: Inst Fondamental Afrique Noire Bull, ser B, v $13, \mathrm{p} 463-475$.

Maurin, L, 1978, Saintes Antiques: Soc Archéol Hist Charente Maritime Press, $150 \mathrm{p}$

Mazière, G, 1983, Informations archéologiques de la circonscription Limousin: La Doue: Gallia Préhist, v 26. p 445-452.

Miallier, D, (ms), 1982, L'usage des détecteurs solides de traces dans le cadre de la datation par thermoluminescence: PhD dissert, Univ Clermont-Ferrand, $121 \mathrm{p}$

Miossec, A, 1977, Le littoral du Pays de Bizerte, étude morphologique: Geol Méditerranéenne, v 4, no.6, p 291-290.

Ortlieb, L, 1980, Cadre géologique du 27ème au 21 ème degré de latitude du Sahara atlantique à l'Holocène: Mém Centre recherche anthropol, prehist, ethnog, v 28, p 9-15.

Oyédé, M, (ms), 1983, Un exemple de sédimentation biodétritique quaternaire dans le domaine margino-littoral en climat tropical humide: le "Lac" Ahémé (Bénin, Afrique de l'Ouest): PhD dissert, Univ Bénin, Univ Dijon, 171 p.

Paskoff, $\mathrm{R}$ and Sanlaville, $\mathrm{P}, 1983$, Les côtes de la Tunisie, variations du niveau marin depuis le Tyrrhénien: Lyon, Maison Orient, $192 \mathrm{p}$.

Pautreau, J P and Robert, P P, 1980: Les Foyers Néolithiques Anciens des Gouillauds au Bois, Ile de Ré, Charente-Maritime: Soc Préhist Fr Bull, v 77, p 123-128.

Philippe, $\mathrm{M}$ and Durand, $\mathrm{M}$, in press, Précisions sur l'âge des remplissages de l'Igue de Barrière: Nouvelles Archives Mus Hist Nat Lyon, v 23, in press.

Philippe, M, Mourer-Cauviré, C, and Evin, J, 1980, Les gisements paléontologiques quaternaires des Causses de Martel et de Gramat (Corrèze et Lot) faune et chronologie: Nouvelles Archives Mus Hist Nat Lyon, v 18, supp, p 57-56.

Piningre, J F, 1979, La sépulture collective mégalithique de Wimereux (Pas-de-Calais) premiers résultats: Rev Nord, v 61, p 950-952.

Planchais, N, 1982, Palynologie lagunaire de l'étang de Mauguio. Paléoenvironnement végétal et évolution anthropique: Pollen et Spores, v 24, no. 1, p 93-118.

Pommepuy, C and Brun, $\mathrm{P}$, in press, La Nécropole de Bucy-le-Long: Rev Archéol Picardie, v 2 , in press.

Poplin, F, Mourer-Chauviré, C, and Evin, J, 1983, Position systématique et datation de Sylviornis neocaleoniae, magapode géant (Aves, Galliformes, Megapodiiae éteint de la Nouvelle Calédonie: Acad Sci (Paris) Comptes rendus, v 297, ser 2, p 301-304.

Pradel, L, 1953, Considérations sur le Périgordien de la Grotte du Fonténioux: Soc Préhist Fr Bull, v 50, p 422-426.

- 1961, La Grotte des Cottés, commune de Saint-Pierre de Maillé, Vienne: Anthropologie, v 65, p 229-270.

1983, Datation par le radiocarbone du Magdalénien VI 2 de la Grotte de Loubresac, commune de Mazerolles (Vienne): Soc Préhist Fr Bull, v 80, p 204.

Pradel, L and Pradel, J H, 1954, Le Moustérien évolué de l'Ermitage, commune de Lussacles-Chateaux (Vienne): Anthropologie, v 58, p 433-443.

1966, La station Paléolithique du Raisse, commune de Brive, Corrèze: Anthropologie, v 70, p 225-253. logie, v 71, p 49-74.

Richards, H G and Broecker, W S, 1963, Emerged Holocene South American shorelines: Science, v 141, p 1044-1045.

Rigaud, J P, (ms), 1982, Le Paléolithique en Périgord: les données du Sud-Ouest Sarladais et leurs implications: PhD dissert, Univ Bordeaux 1, $494 \mathrm{p}$.

Ripotot, R and Seibel, R, 1958, Coup d'oeil sur la préhistoire Comtoise: Ann Lit Univ Besançon, v 20, ser Archéol 5, p 34-47.

Rivallain, J, 1983, Site littoraux du pays Alladian, (Côte d'Ivoire): Premières enquêtes et premiers sondages: Ann Univ Abidjan, ser 1, v 11, p 55-60.

Roubet, C and Matheu, J, 1970, Izimane, station Néolithique de l'Erg oriental, (Sahara algérien): Libyca, v 18, p 67-124. 
Roussot-Laroque, J, 1976, Les civilisations Néolithiques et Chalcolithiques de l'Aquitaine: Préhist Fr, CNRS, v 2, p 338-350.

Rozoy, J G, 1978, Le gisement de l'Allée Tortue, in Les Derniers Chasseurs: Charleville, Rozoy, p 525-534.

Sauzade, G, 1983, Les sépultures du Vaucluse du Néolithique à l'âge du Bronze: Etude Quaternaire, $v 6, p 43$.

Schmider, B, 1979, Un Nouveau faciès du Magdalénien final du Bassin-Parisien: l'industrie du gisement du Pré des Forges, à Marsangy (Yonne), in La fin des temps glaciaires en Europe: Colloque internat CNRS, Paris, no. 271, p, 763-771.

Straus, L, Altuna, J, Clark, G, Gonzales Morales, M, Laville, J, Leroi-Gourhan, A, Menendez de la Hoz, M, and Ortea, J, 1981, Paleoecology at La Riera: Current Anthropology, v 22, p 655-682.

Stuiver, M, 1982, A high-precision calibration of the AD radiocarbon time scale: Radiocarbon, v $24, \mathrm{p} 1-26$.

Tandeau de Marsac, M, 1982, Chapelle et cimetière Médiéval de Champmain (Commune de St-Léonard de Noblat): Travaux archéol Limousine, v 2, p 166.

Tiercelin, J J, (ms), 1981, Rifts continentaux. Tectonique, climats, sédiments. Exemples: La sédimentation dans le Nord du rift Gregory, (Kénya) et dans le rift de 1'Afar (Ethiopie) depuis le Miocène: PhD dissert, Univ Aix-Marseille III, $260 \mathrm{p}$.

Tiercelin, J J, Renaut, R W, Delibrias, G, Le Fournier, J, and Bieda, S, 1981, Late Pleistoecene and Holocene lake level fluctuations in the Lake Bogoria basin, Northern Kenya, Rift Valley: Palaeoecol Africa, v 13, p 105-120.

Toupet, C, 1980, L'enceinte Néolithique de Compiègne (Oise): Préhist Protohist, Champagne-Ardennes, v 4, p 95-108.

Triat-Laval, $\mathrm{H},(\mathrm{ms}), 1979$, Contribution pollenanalytique à l'histoire tardi et postglaciaire de la végétation de la basse vallée du Rhône: PhD dissert, Univ Aix-Marseille III, 343 p.

_ _ 1982, Pollenanalyse de sédiments quaternaires récents du pourtour de l'Etang de Berre: Ecol Mediterranea, v 8, p 97-115.

Van Noten, 1983, Histoire archéologique du Rwanda: Ann Sci Humaines, Tervuren, v 112, $174 \mathrm{p}$.

Vasseur, G, 1911, Une mine de cuivre du Bronze ancien dans les garrigues de l'Hérault: Anthropologie, v 1911, p 419.

Verron, $G$ and Tabbagh, A, 1983, Etude par prospection électromagnétique de trois sites à dépôts de l'Age du Bronze: Soc Préhist Fr Bull, v 80, no. 11-12, p 375-389.

Vié, R, Koutnetzoff, P, and Clot, A, 1983, Nouveau gisement antique à Agos: Lavedan Pays Toy, v 15, p 37-43.

Visset, L, 1982, Nouvelles recherches palynologiques Boréal-Actuel dans les marais de Brière: Ile d'Errand en Saint-Malo de Guersac (L A, France): Assoc Fr Etude Quaternaire Bull, v 1982 , no. 1, p 29-38.

Vital, J, 1981, Recherche archéologique dans les cavités du défilé de Donzère (Drôme): la Grotte de la Chauve-Souris: Nouvelle Archive Mus Hist Nat Lyon, v 19, supp, p 65-66.

Vogel, J C and Waterbolk, H T, 1963, Groningen radiocarbon dates IV: Radiocarbon, v 5, p $166-202$.

_ 166-202. 1967, Groningen radiocarbon dates VII: Radiocarbon, v 9, p 107-155.

1972, Groningen radiocarbon dates X: Radiocarbon, v 14, p 6-110.

Vuillemey, M, in press, La Grotte de la Baume à Gigny-sur-Suran, Jura: Cahiers Centre Recherche Solutré, in press.

Woillard, G, 1975, Recherches palynologiques sur le Pleistocène dans l'Est de la Belgique et dans les Vosges de Lorraine: Acta Lavaniensia, v 14, 118 p.

Young, J A T and Renaut, R W, 1979, Radiocarbon date from Lake Bogoria, Kenya Rift Valley: Nature, v 278, p 243-245. 